\title{
Study of Chromogenic Zwitterionic Chromophores
}

By

Yasaman Aghili

\begin{abstract}
A thesis submitted to
The Faculty of Graduate and Postdoctoral Affairs

In Partial fulfillment of the requirements for the degree of

Master in Chemistry
\end{abstract}

Carleton University

Ottawa, Ontario

August 2013

C Copyright 2013, Yasaman Aghili 


\begin{abstract}
To understand the relationship of structure and chromogenic properties of zwitterionic chromophores, dicyanomethane-based (FDCN and MPCT) and tricyanoquinodimethanebased (PpQDM) chromophores were studied. These chromophores are selected based on the design to have different conjugation lengths and thus have the three primary colors: yellow (FDCN), magenta (MPCT) and cyan (PpQDM). All of the three chromophores exhibited intense absorption in the UV-Vis region with relatively high molar extinction coefficients $(\varepsilon)$ attributed to strong charge transfer (CT) from the negatively charge dicyanomethanide to the positively charged nitrogen in pyridinium.

In this regard, the chromogenic (i.e., color-changing) properties, including solvatochromism, halochromism, and thermo-halochromism of the three chromophores were studied in solution, polymer gel and solid polymer. Distinctive chromogenic features of these zwitterionic chromophores were governed by structural factors, including the strength of the donor and acceptor moieties in addition to the nature of the bridging unit. Thermohalochromic behavior of the chromophores was based on the protonation of the chromophore by acid and consequently deprotonation by heating. Moreover, the colorchanging process could be reversible and irreversible, depending on the nature of a medium. Using malononitrile and a polymer gel as a medium, thermo-halochromic process was irreversible, whereas in a suitable polymer host (i.e., PS, PMMA and PS-co-PMMA), these chromophores showed reversible thermo-halochromic behavior.

Strong charge transfer (CT) of the zwitterionic chromophores in UV-Vis range in addition to their easily CT turned on-off properties eventually led to perform an evaluation based on their potential application as indirect thermo-indicators. FDCN (as the studied chromophore) demonstrated to be capable of reversibly switching on and off the fluorescence of the commercial fluorescent dyes under external stimuli of heat and $\mathrm{pH}$ changes.
\end{abstract}




\section{Acknowledgements}

First of all, I would like to express my cordial gratitude towards my supervisor, Dr. Zhi Yuan (Wayne) Wang for his full support, trust and encouragement during my progress. My true appreciation and respect go to him for his invaluable knowledge, excellent guidance and great advices in the accomplishment of my study and presentation of this thesis.

I am also thankful to Dr. Jane Gao for her valuable guidance, enthusiasm, expertise and encouragement during the past two years. I am greatly thankful to Dr. Wen Hui Hao (Wendy) for all her continuous direction and great knowledge. Her devotion did motivate me on the completion of my Master study and also doing the current research.

I wish to thank my committee members who were more than generous with their expertise and precious time. Special thanks to Dr. Robert C. Burk, Dr. Anatoli Ianoul and Dr. William Willmore agreed to serve on the committee.

I would like to thank all my labmates in our group: Dr. Dou Li (David), Mr. Saif Mia (Hana), Mrs. Di Zhang (Lulu), Mrs. Khama Ghosh and Mr. Sukanta Saha, for their pioneer work, fruitful discussion and cherished friendships making this thesis work an enjoyable experience for me.

I am also thankful to Mr. Keith Bourque and Mr. Tony O'Neil for all their help for NMR and IR measurements and to Mr. Jim Logan for his help with computer affairs.

I would like to give special love and thanks to Mrs. Mastaneh Azad, Mrs. Mehri Hadinezhad, Mr. and Mrs. Naghibalhosseini and all my dear friends, in particular: Afarin, Aida, Arefeh, Azadeh, Bahareh, Diman, Fatemeh, Hiwa, Laleh, Mahsa, Mersa, Neda, Niloofar, Raha, Sarah, Shahrzad, Sharareh, Toktam, Ali, Ali Taba, Bahamin, Behnam, Hamidreza, Mahyar, Mehran, Mehdi, Navid, Omid, Payam, Shahin, and Vahid for being here for me throughout my entire master program. They all have been my best cheerleaders. 
Finally, I want to take this opportunity to appreciate my whole-hearted supportive family and dedicate my thesis work to them. A special feeling of gratitude to my loving parents, Hamidreza Aghili and Nasrin Ganjizadeh Tari for their unconditional love, words of encouragement and tremendous support in every single step of my life even from a long distance. And great feeling of love go to my wonderful brothers, Aryan and Shervin who have never left my side and are very special to me.

"Seek the wisdom that will untie your knot and seek the path that demands your whole being..." -Rumi (Molana) - An Ancient Persian Poet- 


\section{Table of Contents}

Abstract........................................................................................

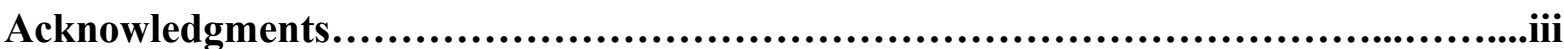

Table of Contents......................................................................v

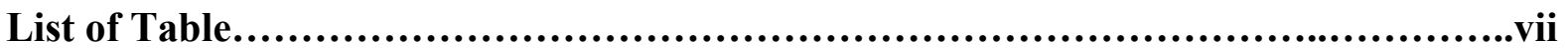

List of Figures...........................................................................

List of Schemes.......................................................................xi

List of Symbols and Abbreviations...................................................xii

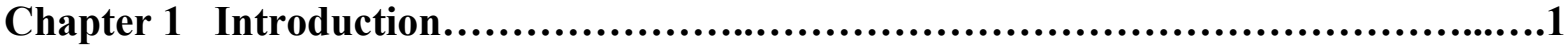

$1.1 \quad$ Zwitterionic Chromophores......................................................

1.2 Properties of Zwitterionic Chromophores....................................

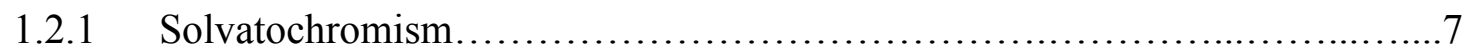

1.2.2 Thermochromism..........................................................

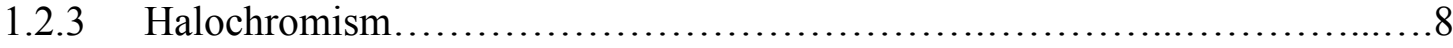

1.2.4 Thermo-halochromism................................................ 8

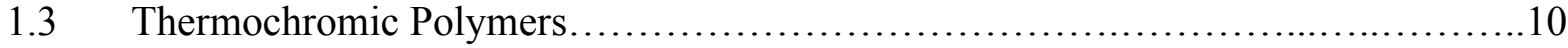

1.3.1 Liquid Crystalline Thermochromic Polymers ..............................11

1.3.2 Leuco Dye-Developer-Solvent Systems.............................12

1.4 Host Polymers........................................................... 15

1.4.1 Polymer Gels........................................................ 15

1.4.2 Guest-Host Polymeric Systems..................................... 16

1.5 Dicyanomethane-based Zwitterionic Chromophores.............................18

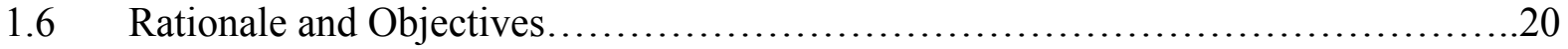

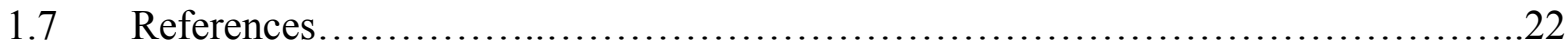

Chapter 2 Synthesis and Characterization of Zwitterionic Chromophores...........25

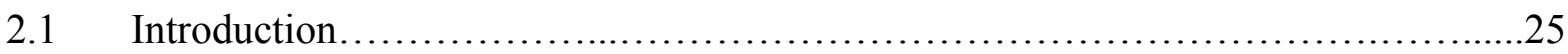

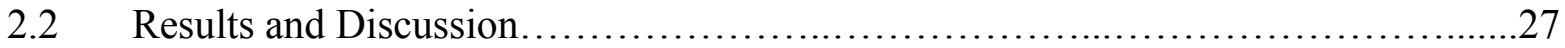

2.2.1 Synthesis and Characterization of MPCT Chromophores.........................27

2.2.2 Synthesis and Characterization of FDCN and PpQDM Chromophore........30

2.2.3 UV-Vis Absorption................................................. 33

2.2.4 Solvatochromic Properties of Zwitterionic Chromophores....................34 
2.2.5 Halochromic Properties of Zwitterionic Chromophores.......................36

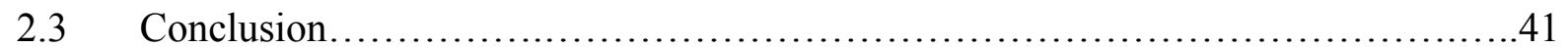

2.4 Experimental Section..................................................41

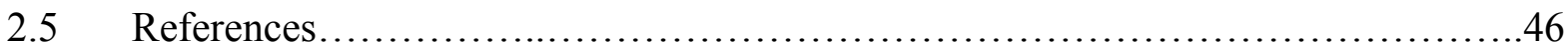

Chapter 3 Thermo-halochromic Properties of Zwitterionic Chromophores..............48

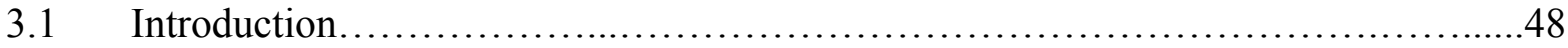

3.2 Results and Discussion....................................................... 51

3.2.1 Thermo-halochromic Properties of Zwitterionic Chromophores in

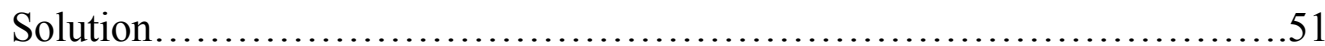

3.2.2 Thermo-halochromic Properties of Zwitterionic Chromophores in Malononitrile..............................................................

3.2.3 Thermo-halochromic Properties of Zwitterionic Chromophores in a Gel Host................................................................60

3.2.4 Thermo-halochromic Properties of Zwitterionic Chromophores in Polymer Hosts.

3.2.5 Application of Zwitterionic Chromophores as Thermo-halochromic Molecular Trigger for Reversible Fluorescence Turn-On/Off..........................66

3.2.5.1 Fluorescence Turn-On/Off Behavior of Perylene/FDCN System......67

3.2.5.2 Fluorescence Turn-On/Off Behavior of Rhodamine 6G/FDCN System.................................................... 70

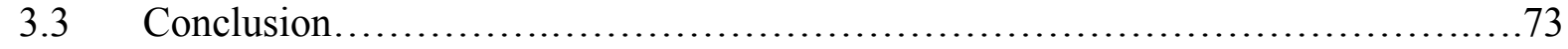

3.4 Experimental Section........................................................ 74

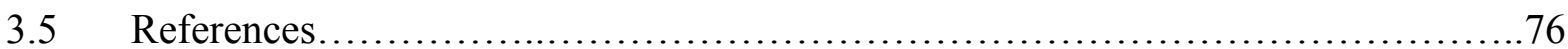

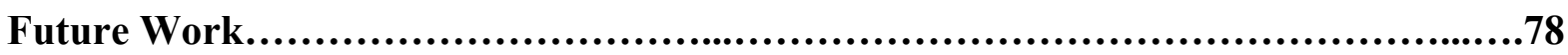

Appendix A ${ }^{1}$ H NMR Spectra for MPCT Synthesis....................................79 


\section{List of Tables}

Table 1.1. Representative structures of betaine and merocyanine zwitterionic

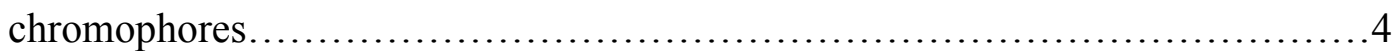

Table 1.2. Zwitterionic chromophore structures containing polyphenylenes bridge with TCF

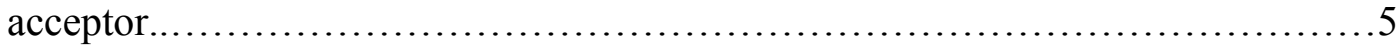

Table 1.3. Representative of $\mathrm{D}^{+}-\pi-\mathrm{A}^{-}$tertiary amine/tricyanoquinodimthane-based (3CNQ)

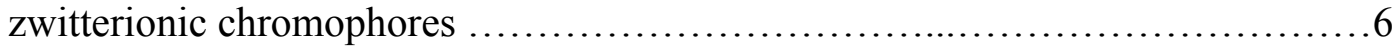

Table 1.4. Different types of thermochromic polymer materials ...........................11

Table 2.1. Molar coefficients $(\varepsilon)$ of FDCN, MPCT and PpQDM in MeCN....................33

Table 2.2. Optical absorption $\left(\lambda_{\max }, \mathrm{nm}\right)$ data for FDCN, MPCT and PpQDM ................34

Table 2.3. Halochromic properties of the zwitterionic chromophores in different acids......40

Table 3.1. Amounts of FDCN, MPCT, and PpQDM in malononitrile with employed acid ...58

Table 3.2. Amounts of zwitterionic chromophores in polystyrene......................63

Table 3.3. FDCN and CSA in polystyrene and poly(methyl methacrylate).................64

Table 3.4. List of commercial fluorescent dyes ......................................66 


\section{List of Figures}

Figure 1.1. Schematic of cholestric liquid crystals structure..........................12

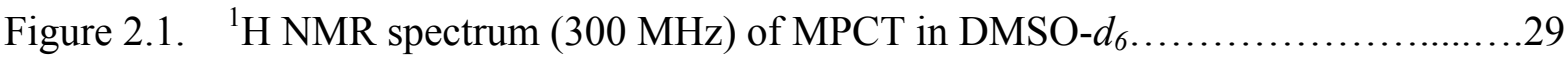

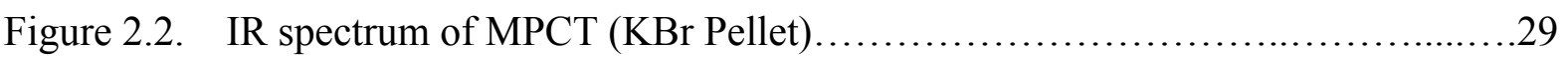

Figure 2.3. Optical absorption spectra of FDCN, MPCT, and PpQDM chromophores in $\mathrm{MeCN}$ solution. Inset: Photographs of the corresponding solutions

Figure 2.4. UV-Vis spectra of MPCT in acetonitrile, acetone and chloroform .............35

Figure 2.5. UV-Vis spectra of FDCN in DMF, DMF/ $\mathrm{H}_{2} \mathrm{O}$ and $\mathrm{HCl} \ldots \ldots \ldots \ldots \ldots \ldots \ldots$

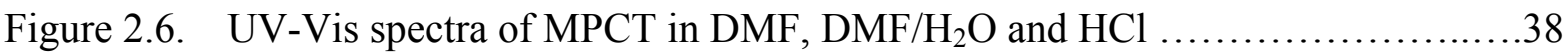

Figure 2.7. UV-Vis spectra of FDCN in $\mathrm{PhCl}$ with a mixture of CSA and DMF ...........39

Figure 3.1. Changes in absorption spectra of FDCN, MPCT, and PpQDM in acetonitrile $\left(\sim 10^{-5} \mathrm{M}\right)$ with CSA $(\mathrm{a}, \mathrm{b}$, and $\mathrm{c})$ and TSA (d, e, and $\left.\mathrm{f}\right)$.

Figure 3.2. Changes in absorption spectra of FDCN in acetonitrile $\left(\sim 10^{-5} \mathrm{M}\right)$ as a mixture with (a, b) 3 equiv. of PpQDM $\left(\sim 10^{-5} \mathrm{M}\right)$ and (c, d) 1 equiv. of PpQDM ( $10^{-5}$ M) with a different amount of CSA and TSA

Figure 3.3. Changes in absorption spectra of FDCN-H $\mathrm{H}^{+}$in acetonitrile $\left(\sim 10^{-5} \mathrm{M}\right)$ upon heating from room temperature to (a) $40{ }^{\circ} \mathrm{C}$, (b) $60{ }^{\circ} \mathrm{C}$, (c) $80{ }^{\circ} \mathrm{C}$ (three successive cycles). Inset: Photographs of the corresponding FDCN solution in acetonitrile

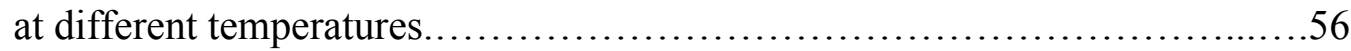

Figure 3.4. Changes in absorption spectra of PpQDM- $\mathrm{H}^{+}$in acetonitrile $\left(\sim 10^{-5} \mathrm{M}\right)$ upon heating from room temperature to (a) $40{ }^{\circ} \mathrm{C}$, (b) $60{ }^{\circ} \mathrm{C}$, (c) $80{ }^{\circ} \mathrm{C}$ (three successive cycles). Inset: Photographs of the corresponding PpQDM solution in acetonitrile at different temperatures .56

Figure 3.5. Irreversible thermo-halochromic behavior of FDCN in malononitrile. Inset: Photographs of the corresponding FDCN solution in malononitrile at three different sates, i.e., liquid state of the bleached (at $35^{\circ} \mathrm{C}$ ) and color return (at $100^{\circ} \mathrm{C}$ ) samples, and solid state of the color return sample (at R.T.) .58

Figure 3.6. Irreversible thermo-halochromic behavior of MPCT in malononitrile. Inset: Photographs of the corresponding MPCT solution in malononitrile at three different sates, i.e., liquid state of the bleached (at $35^{\circ} \mathrm{C}$ ) and color return (at $100^{\circ} \mathrm{C}$ ) samples, and solid state of the color return sample (at R.T.) .58 
Figure 3.7. Irreversible thermo-halochromic behavior of PpQDM in malononitrile. Inset: Photographs of the corresponding PpQDM solution in malononitrile at three different sates, i.e., liquid state of the bleached (at $35^{\circ} \mathrm{C}$ ) and color return (at $100^{\circ} \mathrm{C}$ ) samples, and solid state of the color return sample (at R.T.) .59

Figure 3.8. Irreversible thermo-halochromic behavior of zwitterionic chromophores in a gel host. ...

Figure 3.9. Reversible thermo-halochromic behavior of three zwitterionic chromophores in polystyrene.

Figure 3.10. Thermo-halochromic behavior of FDCN in PS and PMMA

Figure 3.11. Reversible thermo-halochromic behavior of the three zwitterionic chromophores in PS-co-PMMA.

Figure 3.12. Absorption and fluorescence spectra of perylene in chlorobenzene (for emission measurement; $\lambda_{\mathrm{ex}}=410 \mathrm{~nm}$ )

Figure 3.13. Changes in fluorescence spectra of perylene in chlorobenzene $\left(\sim 10^{-6} \mathrm{M}\right)$ via adding FDCN $\left(\sim 10^{-4} \mathrm{M}\right)$ in chlorobenzene and CSA $\left(\sim 10^{-2} \mathrm{M}\right)$ in acetonitrile

Figure 3.14. Use of FDCN-H $\mathrm{H}^{+}$complex to trigger the fluorescence turn-on/off of perylene. Inset: Photographs of the on and off fluorescence emission of perylene 69

Figure 3.15. Changes in absorption spectra of perylene in chlorobenzene $\left(\sim 10^{-6} \mathrm{M}\right)$ via adding FDCN $\left(\sim 10^{-4} \mathrm{M}\right)$ in chlorobenzene and CSA $\left(\sim 10^{-2} \mathrm{M}\right)$ in acetonitrile. Inset: Photographs of the corresponding color changes................................70

Figure 3.16. Absorption and fluorescence spectra of Rhodamine 6G in Chlorobenzene (for emission measurement; $\left.\lambda_{\mathrm{ex}}=490 \mathrm{~nm}\right)$. .71

Figure 3.17. Changes in fluorescence spectra of Rhodamine $6 \mathrm{G}$ in chlorobenzene $\left(\sim 10^{-6} \mathrm{M}\right)$ via adding FDCN $\left(\sim 10^{-4} \mathrm{M}\right)$ in chlorobenzene and CSA $\left(\sim 10^{-2} \mathrm{M}\right)$ in acetonitrile .71

Figure 3.18. Use of FDCN-H $\mathrm{H}^{+}$complex to trigger the fluorescence turn-on/off of Rhodamine 6G. Inset: Photographs of the on and off fluorescence emission of Rhodamine $6 \mathrm{G}$

Figure 3.19. Changes in absorption spectra of Rhodamine $6 \mathrm{G}$ in chlorobenzene $\left(\sim 10^{-6} \mathrm{M}\right)$ via adding FDCN $\left(\sim 10^{-4} \mathrm{M}\right)$ in chlorobenzene and CSA $\left(\sim 10^{-2} \mathrm{M}\right)$ in acetonitrile. Inset: Photographs of the corresponding color changes .72 


\section{List of Schemes}

Scheme 1.1. Schematic presentation of zwitterionic chromophores.........................1

Scheme 1.2. Canonical forms of merocyanines........................................4

Scheme 1.3. Zwitterionic chromophore containing heteroaromatics (e.g. thiophene) in the

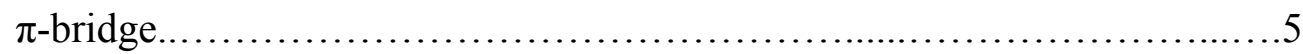

Scheme 1.4. Molecular structure of the pyridinium-N-phenolate betaine dye

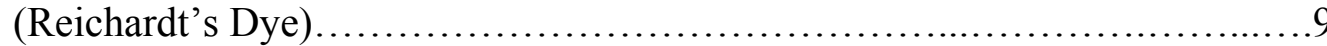

Scheme 1.5. Chemical structures of FDCN, MPCT and PpQDM chromophores...........19

Scheme 2.1. Chemical structure of MPCT chromophore ................................25

Scheme 2.2. Chemical structures of the two synthesized zwitterionic chromophores; FDCM

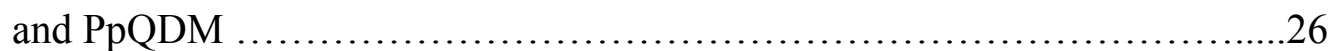

Scheme 2.3. Synthesis of MPCT zwitterionic chromophore $\ldots \ldots \ldots \ldots \ldots \ldots \ldots \ldots \ldots \ldots . .28$

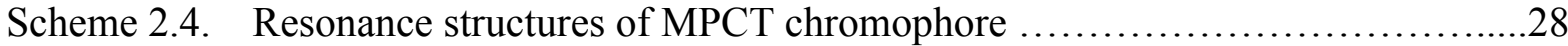

Scheme 2.5. Synthesis of FDCN zwitterionic chromophore ............................. 31

Scheme 2.6. Resonance structures of FDCN chromophore ............................. 31

Scheme 2.7. Synthesis of PpQDM zwitterionic chromophore ...........................32

Scheme 2.8. Resonance structures of PpQDM chromophore ............................32

Scheme 2.9. Protonation and deprotonation of PpQDM ................................. 37

Scheme 3.1. Schematic presentation of protonation-deprotonation process of FDCN .......57

Scheme 3.2. Chemical structure of PEMAGM [poly(ethylene-co-methyl acrylate-co-glycidyl

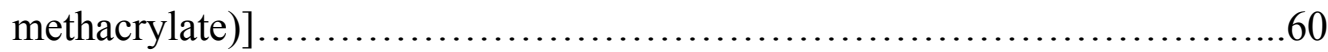

Scheme 3.3. Chemical structures of PS and PMMA ..................................62

Scheme 3.4. Reversible thermo-halochromic behavior of three zwitterionic chromophores in

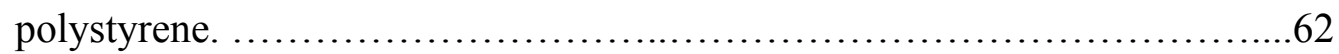

Scheme 3.5. Chemical structure of poly(styrene-methyl methacrylate). ...................64 


\section{List of Symbols and Abbrivations}

$\beta$

$\lambda_{\max }$

3CNQ Tricyanoquinodimethane

BLA Bond-length alternation

CSA Camphorsulfonic Acid

CT Charge transfer

DMF $\quad N, N$-Dimethylformamide

DMSO Dimethyl sulfoxide

FDCN N-alkylpyridinium fluorophenyl dicyanomethane

HOMO Highest occupied molecular orbital

IR Infrared

LUMO Lowest unoccupied molecular orbital

MalCN Malononitrile

$\mathrm{MeCN} \quad$ Acetonitrile

MPCT N-methylpyridinium dicyanomethane thiophene

NIR Near infrared

NLO Nonlinear optical

NMR Nuclear magnetic resonance

PE Polyethylene

PEMAGM Poly(ethylene-co-methyl acrylate-co-glycidyl methacrylate)

$\mathrm{PhCl}$ Chlorobenzene

PL Photoluminescence

PMMA Poly(methyl methacrylate) 
PP Polypropylene

PpQDM Methylpyridine N-methylpyridinium tricynoquinodimethane

PS Polystyrene

PS-co-PMMA Poly(styrene-methyl methacrylate)

TC Thermochromic

TCNQ 7,7,8,8-Tetracyanoquinodimethane

THF Tetrahydrofuran

TSA $\quad p$-Toluenesulfonic acid

UV-Vis Ultraviolet-visible 
"We must not forget that when radium was discovered no one knew that it would prove useful in hospitals. The work was one of pure science. And this is a proof that scientific work must not be considered from the point of view of the direct usefulness of it. It must be done for itself, for the beauty of science, and then there is always the chance that a scientific discovery may become like the radium a benefit for humanity."

-Marie Curie, Lecture at Vassar College, May 14, 1921- 


\section{Chapter 1 Introduction}

This chapter provides background information on zwitterionic chromophores and their properties. Moreover, it gives an overview of various thermochromic polymers.

\subsection{Zwitterionic Chromophores}

A zwitterion refers to a molecule with a positive and negative electrical charge. Zwitterionic chromophores are classified as one of the important types of the intramolecular charge transfer (CT) molecules that bear donor and acceptor moieties at opposite ends of their $\pi$-conjugated systems $\left(\mathrm{D}^{\delta+}-\pi-\mathrm{A}^{\delta-} ; \mathrm{D}\right.$, donor; A, acceptor; $\delta$, degree of $\left.\mathrm{CT}\right)$ (Scheme 1.1). Accordingly, push-pull zwitterionic chromophores can exhibit a highly zwitterionic ground state and a quinoidal excited state.

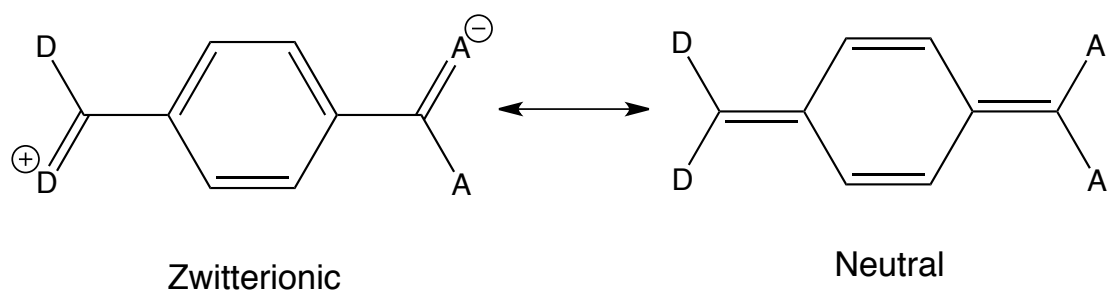

Scheme 1.1. Schematic representation of zwitterionic chromophores.

Zwitterionic chromophores have been investigated for potential applications as thermochromic and halochromic materials. They also show a high hyperpolarizability and therefore are investigated for nonlinear optical (NLO) applications. ${ }^{1-3}$ Moreover Zwitterionic chromophores have been used as solvent polarity indicators due to their large negative solvatochromic effect observed in solvents of increasing polarity. The wide range shifting of the longest wavelength $\mathrm{CT}$ absorption band from the ultraviolet-visible (UV-Vis) to near infrared (NIR) region have made them be a subject of curiosity-driven research in the 
biological, clinical and environmental analyte sensing fields in the recent years. However, despite the significant effort made in this field, a few number of zwitterionic chromophores with small band gap have been explores as NIR molecular fluorescent probes. ${ }^{4}$ To achieve a successful design and development of new dipolar zwitterionic chromophore as potential smart materials and fluorescent probes, understanding the relations between the structure and the corresponding properties are obviously essential.

Distinctive features of zwitterionic chromophores mainly depend on the mutual influence of several factors, such as the strength of the end groups and the connectivity of the $\pi$-electron bridge. In fact, in these molecules, the position of the charge transfer (CT) absorption band depends on the electron affinity of the acceptor moiety and the ionization energy of the donor part. In this regard, a bathochromic (red) shift of the CT absorption band would occur via introduction of electron-withdrawing substituents in the acceptor part, leading to an increase of its electron affinity. ${ }^{5}$

The combination of the two states (i.e., the neutral and the zwitterionic forms) of the zwitterion molecules can be estimated through the use of structural parameters among which, bond length alternation (BLA), ${ }^{6}$ defined as the difference between the average carbon-carbon single and double bond lengths in the polymethine backbone would be dominant. Based on the BLA models, the molecular first hyperpolarizabilities $(\beta)$ values can be increased by decreasing of HOMO-LUMO transition energies or having reduction in the BLA of the entire conjugated systems and employing suitable electron donor and acceptor groups. In fact, the mentioned features would facilitate electron delocalization, which in turn results in small optical gaps in zwitterionic chromophores. 
Furthermore, the nonlinear optical (NLO) properties of zwitterionic chromophores in addition to their applications in different fields of interest have been widely investigated. Accordingly, organic second-order NLO materials have attracted much attention in the past two decades pertaining to their potential applications in telecommunication, optical data storages, optical switches and photonic nano-micro devices. ${ }^{7,8}$ In this regard, the molecular first hyperpolarizabilities $(\beta)$ of zwitterionic chromophores, characterized as the microscopic NLO efficiencies, are attributing to their electronic CT excitation occurring between the ground and the excited states of these molecules.

Based on different types of zwitterionic chromophore, some structures are presented in Table 1.1 to Table 1.3. Betaines (1) and merocyanines (2) are known as zwitterionic chromophores which both show significantly large negative solvatochromism (Table 1.1). ${ }^{5,9,10}$ As an example, based on the table, the longest wavelength $\mathrm{CT}$ absorption band is hypsochromically shifted (i.e., blue shifted) by $357 \mathrm{~nm}$ upon changing the solvent from diphenyl ether $\left(\lambda_{\max }=810 \mathrm{~nm}\right)$ to water $\left(\lambda_{\max }=453 \mathrm{~nm}\right)^{5}$

Merocyanines have been considerably attracted as an important family of zwitterionic chromophores both in industry and academia, during the past years. ${ }^{11,12}$ Based on the represented forms for the two "Neutral" (N) and "Zwitterionic" (ZW) states of zwitterionic chromophores, merocyanines can be also depicted schematically as follows (Scheme 1.2). In fact, the intermediate situation corresponding to the ideal polymethine state, called the "Cyanine Limit" (CL) has been shown here, as well. 
Table 1.1. Representative structures of betaine and merocyanine zwitterionic chromophores.

\begin{tabular}{|l|c|c|c|}
\hline Name & Properties & Ref \\
\hline 2 & & $\begin{array}{c}\Delta \lambda_{\max }=-357 \mathrm{~nm} \\
\left(\lambda_{\text {water }}-\lambda_{\text {ether }}\right)\end{array}$ & \\
\hline & & & \\
\hline
\end{tabular}

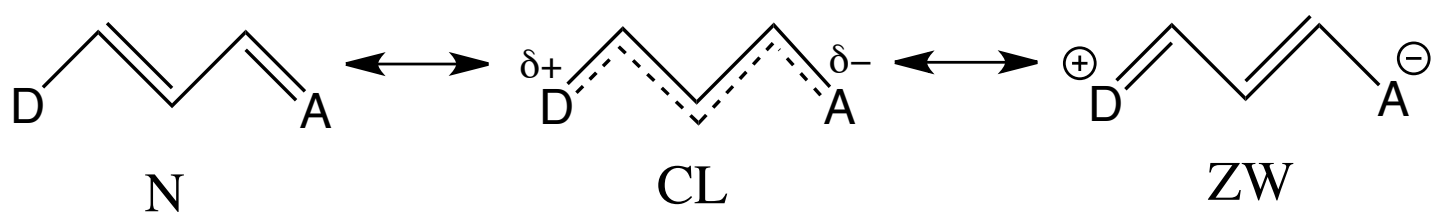

Scheme 1.2. Canonical forms of merocyanines.

The contribution of each of these canonical structures depends either on the nature of the subunits and also the surrounding medium (which can be exemplified by their solvatochromism), ${ }^{13}$ allowing their final optical and photophysical properties to be tuned based on the interplay between the above factors. Phenylpolyene end-capped with aromatic donor and the strong tricyanofuran-based (TCF) acceptor groups $(\mathbf{3}, \mathbf{4})$ are another class of zwitterionic chromophores (Table 1.2). ${ }^{14}$ 
Table 1.2. Zwitterionic chromophore structures containing polyphenylenes bridge with TCF acceptor.

\begin{tabular}{|l|c|c|c|c|}
\hline Name & Properties & Ref \\
\hline 3 & 14 & 14 \\
\hline 4 & & & \\
\hline
\end{tabular}

Another class of zwitterionic chromophores are molecules containing heteroaromatics (e.g. thiophene) in the $\pi$-bridge. The schematic representation of the two ground and excited states of an example of these chromophores has been shown in Scheme 1.3. ${ }^{15}$<smiles>CN1C=CC(=CC=c2ccc(=C(C#N)C#N)s2)C=C1</smiles>

Scheme 1.3. Zwitterionic chromophore containing heteroaromatics (e.g. thiophene) in the $\pi$ bridge. 
Moreover, $\quad \mathrm{D}^{+}-\pi-\mathrm{A}^{-} \quad$ tertiary $\quad$ amine/tricyanoquinodimthane-based $\quad$ (3CNQ) chromophores (Table 1.3) can be mentioned as another zwitterionic family, which have been extensively studies due to their optical properties. ${ }^{3,5,16}$

Table 1.3. Representative of $\mathrm{D}^{+}-\pi-\mathrm{A}^{-}$tertiary amine/tricyanoquinodimthane-based (3CNQ) zwitterionic chromophores.

\begin{tabular}{|l|l|l|l|l|}
\hline Name & Properties & Ref \\
\hline DEMI & & & \\
\hline QQDM & & & \\
\hline
\end{tabular}

In fact, $3 \mathrm{CNQ}$ is one of the best acceptors known to date, which is based on the electron acceptor 7,7,8,8-tetracyanoquinodimethane (TCNQ) and firstly reported in $1960 .{ }^{17}$ TCNQ has been shown to react readily with tertiary amines such as triethyl amine to form stable zwitterionic molecules such as DEMI compound. ${ }^{3,5}$ 


\subsection{Properties of Zwitterionic Chromophores}

The term "chromic" is defined as materials that can change color with the presence of a factor. Specific compounds and materials can change their visible optical properties in response to an external stimulus. In terms of the applied stimulus, they can be classified as thermochromic (temperature), solvatochromic (polarity), halochromic (acidity), photochromic (light), electrochromic (electric field), piezochromic (pressure), ionochromic (ion concentration) or biochromic (biochemical reaction). ${ }^{18}$

\subsubsection{Solvatochromism}

The "solvatochromism" term is used to describe the change of position, intensity, and the shape of the UV-Vis absorption spectrum of the chromophore in the solvent of different polarity. ${ }^{19}$ Positive solvatochromism refers to the absorption of the chromophore, which has a neutral ground state show a bathochromic (red) shift with increasing solvent polarity. On the other hand, when the chromophore has a charge-separated ground state, it leads to negative solvatochromism refers to a hypsochromic (blue) shift of their absorption with increasing solvent polarity. According to the zwitterionic nature of the chromophore, a strong negative solvatochromism effect can be observed as a function of the medium polarity. ${ }^{4}$ Moreover, in the case of negative solvatochromism, increasing the solvent polarity would stabilize the ground state of the zwitterionic chromophores.

\subsubsection{Thermochromism}

Thermochromic (TC) materials, particularly dyes and pigments that are capable of having color changes by varying the temperature, have been a subject of considerable interests in academic research for decades pertaining to their potential applications in a number of industries such as inks, plastics, paints and textiles. TC materials, due to their 
temperature-sensitive features are widely explored as "smart materials" to be practically used in devices such as tuneable light filters, thermal indicators, chemo/biosensors, smart windows, imaging devices and optical switching. ${ }^{18,21}$

\subsubsection{Halochromism}

Term of "halochromism" means the color change which occurs via adding acid (or base, or a salt) to a compound. ${ }^{18}$ In other words, halochromic materials are materials that change color when $\mathrm{pH}$ changes take place (i.e., the external stimulus is $\mathrm{pH}$ ).

The color changes of halochromic substances occur based on the bindings between the chemical and the existing hydrogen and hydroxide ions in solution. The resulting bonds make changes in the conjugate systems of the molecule, or the range of electron flow. This affects the amount of light absorbed, which in turn results in a visible change of color. ${ }^{20}$

\subsubsection{Thermo-halochromism}

Thermo-halochromism is defined as the halochromism effect, which can be influenced by temperature variation. In fact, a rise in temperature may increase or decrease the halochromic shifts, resulting in a positive or negative thermo-halochromism. In an analogous way, when halochromic response is varying with temperature changes, this can be termed as thermo-halochromism. ${ }^{22}$

As previously mentioned, pyridinium-N-phenolate betaine dyes are known as an interesting family of zwitterionic compounds, whose UV-Vis spectra are rather sensitive to its environment. These compounds are capable of sensing the changes of the solvent nature or composition (solvatochromism), the temperature (thermochromism), the nature or concentration of added electrolytes (halochromism) and the medium pressure (piezochromism). ${ }^{23,24}$ 
In this regard, a negative and a positive halochromic behavior of these dyes in solution and in the presence of organic and inorganic salts has been described. ${ }^{25-28}$ In one study, ${ }^{22}$ variations of the position of the CT band of a pyridinium-N-phenolate betaine; i.e., Reichardt's Dye (Scheme 1.4) were investigated in a number of solvents including methanol, 1-propanol, 1-butanol, acetonitrile $(\mathrm{MeCN})$ and $N, N$-dimethylformamide (DMF), in the presence of increasing concentration of NaI salt, at $15{ }^{\circ} \mathrm{C}$ and $55^{\circ} \mathrm{C}$. In all cases, increasing the cation concentration resulted in a hypsochromic shift (blue shift) of the CT band.<smiles></smiles>

Scheme 1.4. Molecular structure of the pyridinium-N-phenolate betaine dye (Reichardt's Dye).

The thermo-halochromism of the mentioned dye is in fact the result of the interplay of three interactions occurs in the solution: a dye-cation, a dye-solvent and a cation-solvent interaction, and of how temperature affects these interactions. A simplified picture of this three-component system can rationalize the observed trend, based on the two following dissociation processes, in which $\mathrm{PhO}^{-}$(solv) stands for the solvated pyridinium-N-phenolate betaine dye and the solvated $\mathrm{Na}^{+}$is actually regarded as the cation component. 


$$
\begin{aligned}
& \mathrm{NaI} \text { (solv) } \longleftrightarrow \mathrm{Na}^{+} \text {(solv) }+\mathrm{I}^{-} \text {(solv) } \\
& \mathrm{PhONa} \text { (solv) } \longleftrightarrow \mathrm{PhO}^{-} \text {(solv) }+\mathrm{Na}^{+} \text {(solv) }
\end{aligned}
$$

Any factor that favors the NaI dissociation (1) will result in the higher concentration of free $\mathrm{Na}^{+}$(solv) and shift the equilibrium (2) to the left and therefore, increasing the dyecation association.

Moreover, further studies showed that in less polar solvents, the NaI molecules would require higher temperature as the solvating power of the $\mathrm{Na}^{+}$and $\mathrm{I}^{-}$ions decrease in such cases. Regarding the solvent polarity as a measurement of the strength for salt dissociation, the betaine molecule exhibited a negative thermo-halochromism in polar solvents like methanol or DMF, whilst in less polar solvents like 1-propanol, 1-butanol and MeCN it showed a positive thermo-halochromism.

The above analysis is in fact based on the systems in which the solvent and the salt are different chemical species. Study of the thermo-halochromism of dyes in ionic liquids (IL) has recently received increasing attention, since ionic liquids can act both as solvent and electrolyte, so that such studies can be considered an ultimate description of the thermohalochromism behavior of a dye. ${ }^{22}$

\subsection{Thermochromic Polymers}

In attempting to design all organic/polymeric materials as active components to incorporate zwitterionic chromophores, a number of criteria need to be addressed and certain parameters have to be traded. ${ }^{14}$ Thermochromism can appear in different classes of polymers, including thermoplastics, gels, inks, paints and coatings. ${ }^{20}$ As one particular case of thermoplastic polymers, fiber materials should be mentioned. Thermochromic cellulose 
fibers, which were fabricated by incorporating the commercial thermochromic pigments, have been reported. ${ }^{29}$ To fabricate the thermochromic cellulose fibers, firstly the thermochromic pigments have been dispersed into a cellulose spinning solution and then followed by spinning fibers from the modified solution. The obtained fibers are reported to exhibit a thermochromic color intensity change from magenta to colorless at a temperature of 32.7 to $32.9^{\circ} \mathrm{C}$, switching range of $0.2^{\circ} \mathrm{C}$.

Thermochromic effects can be caused by the polymer itself or via an embedded thermochromic additive. A list of different types of thermochromic polymer materials is displayed in Table 1.4. ${ }^{20}$

Table 1.4. Different types of thermochromic polymer materials.

\begin{tabular}{|c|c|}
\hline Polymers Thermochromic themselves & $\begin{array}{c}\text { Polymer Materials Doped with } \\
\text { Thermochromic Additives }\end{array}$ \\
\hline Liquid Crystalline Polymers & Leuco Dye-developer Solvent Systems \\
\hline Conjugated Polymers & Dyes embedded in Polymeric Hosts \\
\hline $\begin{array}{c}\text { Swollen Gel Network of Conjugated } \\
\text { Polymers }\end{array}$ & Conjugated Polymers / Polymer Blends \\
\hline
\end{tabular}

\subsubsection{Liquid Crystalline Thermochromic Polymers}

The potential application of liquid crystals with helical superstructures (known as cholestric liquid crystals) for optical data recording in the realm of information technology is one of the main driving forces for their further development. ${ }^{30}$ 
The cholestric liquid crystals are a type of liquid crystals with a helical structure in which the crystals are organized in layers with a director axis that varies with layers (Figure $1.2){ }^{20}$

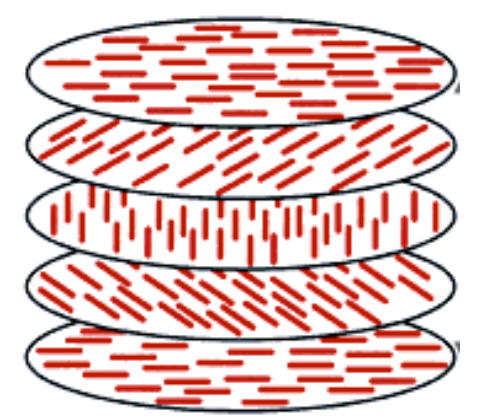

Figure 1.2. Schematic of cholestric liquid crystals structure.

In this regard, two methods are used to fix the helical superstructures: freezing in the molecular structure by cooling into a glassy state or carrying out a crosslinking reaction whereby a polymer network structure is formed. ${ }^{31,32}$ Depending on temperature, different colors can be appeared which on fast cooling into glassy state, they can be fixed. However with these materials it would be rather difficult to create patterns with different colors since several hours will be required to gain an equilibrium state in the helical structure since after the temperature changes occur within the cholestric phase. ${ }^{20}$

\subsubsection{Leuco Dye-Developer-Solvent Systems}

Leuco dye-developer-solvent systems can be mentioned as materials in which the polymer is doped with thermochromic additives. These systems include an electron-donating chromogenic compound (leuco dye), an electron acceptor (developer) and a matrix component (solvent). ${ }^{20}$ Phenylmethane and fluoran derivatives bearing a lactone ring moiety are known as the most electron-donating chromogenic compounds. In fact, in the lactone 
ring-closed state, these so-called leuco dyes or color formers are either colorless or light yellow color. Therefore, formation of a zwitterionic structure occurs via a ring-opening reaction of the lactone ring resulting by the presence of an electron-accepting developer. As a result of this reaction, the conjugated $\pi$-electron system of the dye will be extended and the longest wavelength absorption peak will shift from the UV to the visible range and thus, the dye will become colored. The presence of an appropriate solvent component enabled the color-forming reaction to be reversible with temperature. In the solid state, the leuco dyedeveloper-solvent systems are colored, whereas they transform into a colorless liquid on heating above their melting temperature. The solvent function as an inhibitor of the colorforming reaction, whilst it obviously does not interfere with the formation of the color in the solid state. $^{20}$

Despite knowing the color-forming reaction of leuco dyes for more than 50 years, the detailed molecular mechanism is still unclear to some extent. A proton-triggered ring opening/ring-closing mechanism was initially revealed as the only model discussed in the literature, until about 15 years ago. Based on this model, the effectiveness of the developer is measured according to its acidity in order to drive the ring-opening/ring-closing equilibrium of leuco dyes towards their ring-open color state. However, this simple model was not able to explain the experimental results, in many cases. ${ }^{33}$

In general, reversible thermochromic systems consist of at least three components: a leuco dye, a developer and a solvent. The additional third component actually makes the system even more complex. In most of the solvent components reported in literatures, long hydrocarbon chain bearing a polar end-group on one side of the chain is present. The presence of the hydrocarbon chain and the polar end group allow disperse-disperse 
interaction and a polar-polar interaction, respectively. In leuco-dye developer systems, the latter interaction especially occurs between the solvent and the developer. The reversible thermochromic effect of these systems has been explained based on a competition between dye-developer and solvent-developer interactions. ${ }^{34,35}$

In the solid state the dye-developer interactions dominate and colored dye-developer complexes are formed. On the other hand, the solvent-developer interactions prevail on heating from the solid into the molten state. Therefore, decolorization of the system occurs once the dye-developer complexes are destroyed. Corresponding melting compounds composed of two developer molecules and one solvent molecule are observed in the binary phase diagrams between different developers and solvents. ${ }^{34,36}$

On the other hand, although there is a number of examples for reversible thermochromic leuco dye-developer-solvent systems, a few examples of two-component systems have been reported, in which one component has the both function of solvent \& developer. ${ }^{37,38}$ Comparing to the three-component leuco dye-developer-solvent systems, the reversible thermochromic two-component systems are colorless in the solid state and colored in the molten state. One example of these systems is a mixture of fluoran leuco dye with octadecylphosphine acid in which on heating the mixture (above $100^{\circ} \mathrm{C}$ ), it completely melts and becomes colored, whereas cooling at low rates results in a crystallization of the mixture at about $70{ }^{\circ} \mathrm{C}$ following by decolorization. Other experiments in the realm of leuco dyedeveloper-solvent systems have confirmed the possibility of a combination mechanism of the ring-opening/ring-closing of the leuco dyes including the proton-transfer complexes. ${ }^{20}$ 


\section{$1.4 \quad$ Host Polymers}

\subsubsection{Polymer Gel}

Based on the weight, gels are mostly liquid, whilst they behave like solids due to their three-dimensional cross-linked networks within the liquid phase. In other words, polymeric gel networks are solvent-filled matrices, formed by cross-linking reactions in which weak physical interactions or strong chemical interactions can be present. Polymeric gel networks have been reported to be utilized in different fields of interest, such as drug delivery systems, coatings, food and cosmetics, or as matrix structures in tissue engineering and smart materials. ${ }^{39,40}$

Accordingly, Sol-gel process is also widely known as one particular strategy, which involves the transition of a solution system from a liquid "sol" into a solid "gel" phase. By employing the sol-gel process, it would be possible to fabricate advanced materials, including ceramics and organic-inorganic hybrids. ${ }^{41}$

Polymer gels provide a useful platform for studying the thermochromic properties, including different strategies such as introducing indicator dyes into the polymeric gel media. ${ }^{42}$ In this study, pyridinium N-phenolate betaine dye (Reichart's Dye), which has been introduced as a well-known compound due to its strong solvatochromism exhibited significant thermochromic properties via embedded in an aqueous polyvinyl gel network. In this case, the color changes occurred reversibly from colorless to a deep violet via heating. The observed thermochromic behavior can be explained by a temperature-induced shift of the proton-transfer equilibrium between the phenolate and the phenol forms of the dye molecules in the microenvironment of the employed polymeric gel network. Thermochromic properties of this dye have been widely studied according to its highly zwitterionic ground state. 
In this regard, a hydrogel (i.e., a network of polymer chains that are hydrophilic) can act as a medium in thermochromic materials for incorporated dye molecules. ${ }^{43}$ Successive studies have been carried out by Seeboth et al. based on embedding dyes with thermochromic properties in hydrogel networks. ${ }^{42-45}$ First example of reversible thermochromic of phenolsubstituted dyes embedded in transparent hydrogels has been reported in $1998,{ }^{44}$ in which the same proton-transfer equilibrium was dominant in the system.

Thermochromic phenomenon in polymeric gel media can be expected to have different applications including thermal sensors, smart windows, etc. In some cases, this color change is permanent (i.e., irreversible). Based on irreversible behavior, engineered thermochromic systems have been designed to be utilized in different areas such as monitoring labels (i.e., temperature sensing stickers) utilized to ensure food safety during storage and transportation. ${ }^{46}$

The incorporation of zwitterionic chromophores into an appropriate matrix is quite a challenging task. During the past years, polymeric gel materials have become an interesting field of research, mainly as a host component for NLO applications of zwitterionic guest compounds, based on the very specific requirements of the zwitterionic nature of the chromophores upon incorporation in a solid matrix. The high versatility of preparing gel networks from polymer solutions offers a wide range of possibilities to design materials in terms of various structures and functionalities. ${ }^{14,20}$

\subsubsection{Guest-Host Polymer Systems}

Investigations have shown that thermochromic chromophores can be successfully introduced into polymer matrices and the reversible thermochromic effects can be retained. 
Accordingly, combinations of chromophores and solvents in polymeric media such as polyethylene (PE) and polypropylene (PP) have been developed. ${ }^{47}$

Guest-host polymeric systems are known as the simplest approach in which the chromophores, as the guest, are physically incorporated into a polymer host. In this regard, structural features of the zwitterionic molecules, synthetic expediency, and transparency of the polymeric host component and its compatibility with the doped guest chromophore molecules in addition to thermal properties and photostability of the components should be mentioned. ${ }^{48}$ Combination of different polymer matrices and chromophores can be facilitated via this approach, however it suffers from a number of drawbacks including low concentration of chromophores that can be doped in the polymer without aggregation. ${ }^{48,49}$

In one example, guest-host polymeric thermochromic materials have been prepared via incorporation of cyano-substituted dyes into a polyamide medium. ${ }^{50}$ Dyes with reversible thermochromic behavior have been also identified in which emulsion polymerization has been utilized to encapsulate the dye molecules. In this regard, thermochromic coatings used as the paints for buildings have been designed through blending the prepared microencapsulated thermochromic dyes with ordinary white paints. The colors of these systems can be reversibly changed from warm tones to cool tones upon increasing temperatures. $^{51}$

Thermochromic displays can be mentioned as one candidate for chromatic applications. In the past recent years, novel polymer-based display types have been fabricated from thermochromic compounds. ${ }^{52,53}$ In addition, polymer-based thermochromic systems have been also reported with a potential application as smart windows. ${ }^{20}$ 
In some cases of incorporating a thermochromic material into a polymer matrix, both components build separate phase and their phase structures would not be influenced by the other component. In this regard, microencapsulated leuco dye-developer-solvent systems, inorganic pigments and conjugated polymers are suitable candidates for this purpose, among which leuco dye-developer complexes are the most important systems to achieve thermochromic properties for different polymer materials by introducing a separate phase of the thermochromic system in a non-thermochromic polymer matrix. Due to their importance for the development of thermochromic materials, the properties are of these systems (i.e., the leuco dye-developer complexes) have been discussed in much more details in the literatures. $^{20}$

\subsection{Dicyanomethane-based Zwitterionic Chromophores}

The current research has been focused on the study of the properties of two novel dipolar zwitterionic chromophores; a dicyanomethane-based one (i.e., FDCN with a trivial name of $\mathrm{N}$-alkylpyridinium fluorophenyl dicyanomethane), and a pyridinium tricynoquinodimethane-based one (i.e., PpQDM with a trivial name of methylpyridine Nmethylpyridinium tricynoquinodimethane), previously synthesized in our group. ${ }^{54}$ In addition, a dicyanomethane-thiophene-based zwitterionic chromophore, (i.e., MPCT with a trivial name of $\mathrm{N}$-methylpyridinium dicyanomethane thiophene) with a conjugation length between FDCN and PpQDM has been synthesized in this research via a facile and clean synthesis route based on a work carried out by Abbotto et al. in $1997 .{ }^{55}$ The major goal of this research was the characterization of the three mentioned zwitterionic chromophores (Scheme 1.5) with respect to their intrinsic halochromic properties depending on $\mathrm{pH}$ alternation and solvatochromic properties depending on solvent polarity changes (Chapter 2). 
Moreover, the thermo-halochromic properties of the mentioned chromophores depending on both $\mathrm{pH}$ and temperature changes were investigated in solution phase as well as by primarily incorporating them into some appropriate polymeric and gel media in addition to a final study as the thermo-indicator carried on their CT on-off properties displayed by an indirect photoluminescence (PL) off-on of the commercial dyes (Chapter 3).<smiles>C[n+]1ccc(-c2ccc(C(C#N)C#N)s2)cc1</smiles><smiles>Cc1ccnc(-c2cc(/C=C(\C#N)c3ccc([C-](C#N)C#N)cc3)cc[n+]2C)c1</smiles>

\section{PpQDM}

Scheme 1.5. Chemical structures of FDCN, MPCT and PpQDM chromophores.

Accordingly, the zwitterionic chromophores with tunable conjugation lengths have been resulted in providing the three primary colors; i.e., yellow (FDCN), Magenta (MPCT), and cyan (PpQDM). In this regard, the potential designing of a full-spectrum color indication based on the chromophores combination can be investigated in future, pertaining to the subtractive color theory. On the other hand, strong charge transfer (CT) of the mentioned zwitterionic chromophores in UV-Vis range in addition to their easily CT turned on-off 
properties resulting in reversible thermo-halochromism behaviors leading to potential application as indirect thermo-indicators.

\section{6 Rationale and Objectives}

As previously mentioned, dipolar zwitterionic chromophores have distinguished features mainly depend on the mutual influence of the strength of the donor and the acceptor extremities of the molecules in addition to the connectivity of the $\pi$-electron bridge. Pertaining to introduction of electron-withdrawing substituents in the acceptor part, a bathochromic (red) shift of the $\mathrm{CT}$ absorption band would occur resulting in an increase of its electron affinity.

This research is particularly interested in development and study of dipolar zwitterionic chromophores with the potential of thermo-halochromic applications with emphasis on their strong absorption and chromic properties. In order to gain a comprehensive knowledge of zwitterionic chromophores for potential applications, it is essential to better understand the structure-property relationship, in particular the strength of the donor and acceptor moieties and the conjugation length. Therefore, the main objectives of this research are:

1) To synthesize three zwitterionic chromophores, i.e., FDCN (yellow), MPCT (magenta) and PpQDM (cyan), having different conjugated lengths (labelled as short, medium, and long), colors, and solvatochromic and halochromic properties.

2) To investigate the halochromism and thermo-halochromism of these chromophores as a guest in three different media: solution, polymer and gel network. 
3) To demonstrate the use of FDCN as a trigger to switch on and off the fluorescence of commercial fluorescent dyes under external stimuli of heat and $\mathrm{pH}$ changes. 
1.7 References

(1) Lebeau, B.; Innocenzi, P. Chem. Soc. Rev. 2011, 40, 886..

(2) Bell, N. A.; Crouch, D. J.; Simmonds, D. J.; Goeta, A. E.; Gelbrich, T.; Hursthouse, M. B. J. Mater. Chem. 2002, 12, 1274.

(3) Ashwell, G.J. Thin Solid Films 1990, 186, 155.

(4) Papadopoulos, M. G.; Sadlej, A. J.; Leszczynski, Non-Linear Optical Properties of Matter: From molecules to condensed phases Springer, 2006.

(5) Reichardt, C.; Chem. Rev. 1994, 94, 2319.

(6) Marder, S. R.; Perry, J. W.; Bourhill, G.; Gorman, C. B.; Tiemann, B. G.; Mansour, K. Science 1993, 261, 186.

(7) Kajzar, F.; Lee, K. S.; Jen, A. K-Y. Adv. Polym. Sci. 2003, 161, 1.

(8) Dalton, L. R.; Sullivan, P.; Bale, D. H. Chem. Rev. 2010, 100, 25.

(9) Baroo, A.; Zerner, M. C. Chem. Phys. 1995, 196, 423.

(10) Dulcic, A.; Flytzanis, C. Opt. Commun. 1978, 25, 402.

(11) Andreu, R.; Galan, E.; Orduna, J.; Villacampa, B.; Alicante, R.; Navarrete, J. T. L.; Casado, J.; Garin, J. Chem. Eur. J. 2011, 17, 826.

(12) (a) Fabian, J.; Hartmann, H. Light Absorption of Organic Colorants, Springer, 1980.

(b) Kulinich, A. V.; Ishchenko, A. A. Russ. Chem. Rev. 2009, 78, 141.

(13) Reichardt, C. Solvents and Solvents Effects in Organic Chemistry, VCH, Weinheim, 1994.

(14) Kay, A. J.; Woolhouse, A. D.; Zhao, Y.; Clays, K. J. Mater. Chem. 2004, 14, 1321.

(15) Abbotto, A.; Beverina, L.; Bradamante, S.; Facchetti, A.; Klein, C., Pagani, G. A.; Redi-Abshiro, M.; Wortmann, R. Chem. Eur. J. 2003, 9, 1991.

(16) Ashwell, G. J., Bloor, D. Organic Materials for Nonlinear Optic III, Royal Society of Chemistry, 1993.

(17) Acker, D. S.; Harder, R. J.; Hertler, W. R.; Mahler, W.; Melby, L. R.; Benson, R. E.; Mochel, W. E. J. Am. Chem. Soc.1960, 82, 6408.

(18) Bamfield, P. Chromic Phenomena: Technological Applications of Colour Chemistry Royal Society of Chemistry, Cambridge, 2001.

(19) Innocenzi, P.; Miorin, E.; Brusatin, G. Chem. Mater. 2002, 14, 3758. 
(20) Seeboth, A.; Lotzsch, D. Thermochromic Phenomena in Polymers, Smithers Rapra Technology Limited, 2008.

(21) Qian, G.; Wang, Z. Y. Adv. Mater. 2012, 24, 1582.

(22) Rezende, M. C.; Aracena, A. Spectrochimica Acta Part A: Molecular and Biomolecular Spectroscopy 2012, 98, 18.

(23) Reichardt, C.; Welton, T. Solvents and Solvent Effects in Organic Chemistry, fourth ed., Wiley-VCH, Weinhemin, 2011.

(24) Reichardt, C. Pure Appl. Chem. 2004, 76, 1903.

(25) Gageiro, V.; Aillon M.; Rezende, M.C. J. Chem. Soc. 1992, 88, 201.

(26) Zanotto, S.P.; Scremin, M.; Machado, C.; Rezende, M.C. J. Phys. Org. Chem. 1993, $6,637$.

(27) Ramirez, C.B.; Carrasco, N.; Rezende, M.C. J. Chem. Soc. 1995, 91, 3839.

(28) Rezende, M.C.; Dominguez, M.; Aracena, A. Spectrochim. Acta 2012, 87, 61.

(29) Rubacha, M. Polym. Adv. Tech. 2007, 18, 323.

(30) Tamaoki, N. Adv. Mater. 2001, 13, 1135.

(31) Yamagishi, T. A.; Sixou, P. Polymer 1995, 36, 2315.

(32) Hikmet, R. A. M.; Polesso, R. Adv. Mater. 2002, 14, 502.

(33) Burkinshaw, S. M.; Griffiths, J.; Towns, A.D. J. Mater. Chem. 1998, 8, 2677.

(34) MacLaren, D. C.; White, M. A. J. Mater. Chem. 2003, 13, 1701.

(35) Luthern, J.; Peredes, A. J. Mater. Sci. Lett. 2003, 22, 881.

(36) MacLaren, D. C.; White, M.A. J. Mater. Sci. 2005, 40, 669.

(37) Naito, K. Appl. Phys. Lett. 1995, 67, 211.

(38) Naito, K. J. Mater. Chem. 1998, 8, 1379.

(39) Skrzeszewska, P. J.; Sprakel, J.; Wolf, F. A. D.; Fokkink, R.; Stuart, M. A. C.; Gucht, J. V. D. Macromolecules 2010, 43, 3542.

(40) Mpoukouvalas, A.; Li, W.; Graf, R.; Koynov, K.; Matyjaszewski, K. Macro. Lett. 2013, 2, 23.

(41) Brinker, C. J. Sol-Gel Science: The Physics and Chemistry of Sol-Gel Processing, Academic Press, 1990.

(42) Seeboth, A.; Kriwanek, J.; Vetter, R. Adv. Mater. 2000, 12, 1424.

(43) Seeboth, A.; Kriwanek, J.; Vetter, R. J. Mater. Chem. 1999, 9, 2277. 
(44) Seeboth, A.; Holzbauer, H.-R. Int. J. Restoration Buildings Monuments 1998, 4, 507.

(45) Fischer, T.; Holzbauer, H.-R.; Seeboth, A. Mater. Sci. Eng. Technol. 1999, 30, 473.

(46) Rahman, F.; Khokhar, A. Z. Appl. Phys. A 2008, 94, 405.

(47) Seeboth, A.; Ruhmann, R.; Mühling, O. Materials 2010, 3, 5143.

(48) Jen, A. K-Y.; Wong, K. Y.; Rao, V. P.; Drost, K. J. Electron. Mater. 1994, 23, 653.

(49) Cai, Y. M.; Jen, A. K-Y.; Appl. Phys. Lett. 1995, 117, 7295.

(50) Kunzelman, J.; Crenshaw, B. R.; Weder, C. J. Mater. Chem. 2007, 17, 2989.

(51) Ma, Y.; Zhu, B.; Wu, K. J. Coatings Technol. 2000, 72, 67.

(52) Yarimaga, O.; Im, M.; Choi, Y-K. Macro. Research 2010, 18, 404.

(53) Liu, L.; Peng, S.; Wen, W.; Sheng, P. Appl. Phys. Lett. 2007, 90, 213508.

(54) Hao, W. H. "Development of Zwitterionic Chromophores and Nonlinear Optical Polymers for Electro-Optic and Near Infrared Chemosensor Applications", Ph. D. Thesis, Carleton University, 2011.

(55) Abbotto, A.; Bradamante S.; Facchetti, A.; Pagani, G. A. J. Org. Chem. 1997, 62, 5755. 


\section{Chapter 2 Synthesis and Characterization of Zwitterionic Chromophores}

\subsection{Introduction}

Development of thiophene-derived zwitterionic chromophores with enhanced thermal stability was triggered about two decades ago. ${ }^{1,2}$ Within these compounds, particularly large molecular hyperpolarizabilities were found once dicyanovinyl and tricyanovinyl acceptors are present in the chromophore structure. In fact, this class of chromophores have attracted attention during the past years, due to their zwitterionic nature, which leads to high groundstate dipole moments and large negative solvatochromism. ${ }^{3,4}$ With respect to this connection, it has been shown ${ }^{5}$ that the pyridine moiety is among the highest ranked electron-donor groups and can efficiently promote charge transfer to the acceptor moiety. ${ }^{6,7}$ In this regard, a zwitterionic chromophore with a trivial name of N-methylpyridinium dicyanomethane thiophene (MPCT) was synthesized in this thesis research via a facile synthetic route based on a work carried out by Abbotto et al. in $1997 .{ }^{8}$ This compound is known as a push-pull system, in which there is an $\mathrm{N}$-alkylpyridine moiety as the donor and a dicyanomethane as the acceptor group. The terminal polar functions are spaced by a thiophene-based moiety. Chemical structure of MPCT is depicted in Scheme 2.1.

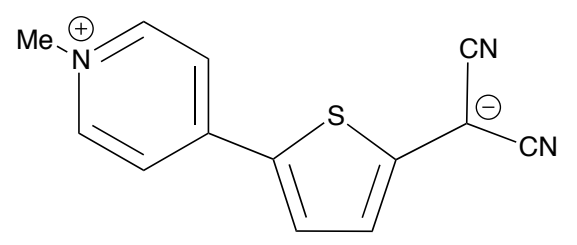

Scheme 2.1. Chemical structure of MPCT chromophore.

Moreover, to understand the structure-property relationship of zwitterionic chromophores and realize the full potential of these chromophores, this chapter describes the 
syntheses of two series of zwitterionic chromophores, i.e., FDCN and PpQDM chromophores (Scheme 2.2) designed and prepared in our group in 2011. ${ }^{9-11}$ The synthetic details in addition to characterizations of the two chromophores including the X-ray crystallographic analysis, thermal stability, negative solvatochromic and microscopic NLO properties have been also reported previously. ${ }^{11}$

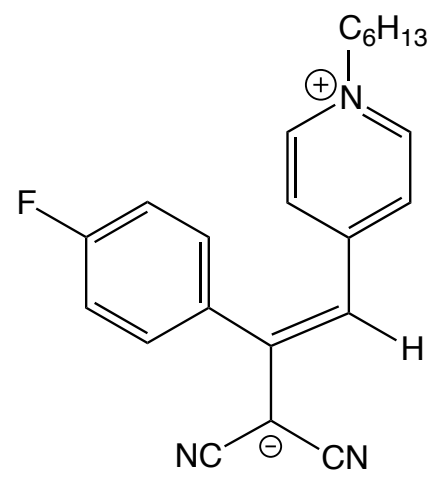

FDCN<smiles>Cc1ccnc(-c2cc(/C=C(\C#N)c3ccc(C(=O)C#N)cc3)cc[n+]2C)c1</smiles>

PpQDM

Scheme 2.2. Chemical structures of the two synthesized zwitterionic chromophores.

In addition to study the solvatochromic behavior of the three mentioned zwitterionic chromophores, these chromophores have been also characterized for their potential halochromic properties (depending on $\mathrm{pH}$ ) in order to understand the structure-property relationship of zwitterionic chromophores with different conjugation lengths; i.e., FDCN (yellow color with 6 carbon-carbon double bonds in conjugation), MPCT (magenta color with 8 carbon-carbon double bonds in conjugation, and PpQDM (cyan color with 10 carboncarbon double bonds in conjugation), considering a potential application as full-spectrum thermo indicators in future. 


\subsection{Results and Discussion}

\subsubsection{Synthesis and Characterization of MPCT Chromophores}

MPCT was synthesized in this thesis research as a zwitterionic chromophore based on a work reported in $1997 .{ }^{8}$ This compound in fact consists of an $\mathrm{N}$-alkylpyridine ring (the donor) bonded to a negatively charged dicyanomethane (the acceptor) through a $\pi$-conjugated thiophene spacer. The synthetic route to MPCT and the resonance structure of MPCT zwitterionic chromophore are depicted in Schemes 2.3 and 2.4, respectively.

MPCT is readily soluble in polar solvents, such as MeCN, DMF, DMSO, methanol and acetone and essentially not soluble in less polar solvents such as chloroform and THF.

The ${ }^{1} \mathrm{H}$ NMR spectrum of the MPCT chromophore with the assigned peaks is shown in Figure 2.1. The observed proton resonances (ppm) at $8.26(2 \mathrm{H}, \mathrm{d}, \mathrm{J}=7.0 \mathrm{~Hz}), 7.92(1 \mathrm{H}, \mathrm{d}$, $\mathrm{J}=4.4 \mathrm{~Hz}), 7.62(2 \mathrm{H}, \mathrm{d}, \mathrm{J}=7.0 \mathrm{~Hz}), 6.41(1 \mathrm{H}, \mathrm{d}, \mathrm{J}=4.4 \mathrm{~Hz})$, and $3.96(\mathrm{~s}, 3 \mathrm{H})$ are consistent with the structure of MPCT.

Figure 2.2 displays the IR spectrum of MPCT. The peaks at 2178 and $2160 \mathrm{~cm}^{-1}$ are characteristic of the cyano groups connected to an electron-rich group with a negative charge. Moreover, the melting point of the synthesized chromophore was measured $\left(\mathrm{mp}>240^{\circ} \mathrm{C}\right)$, being consistent with the literature value. ${ }^{8}$ 

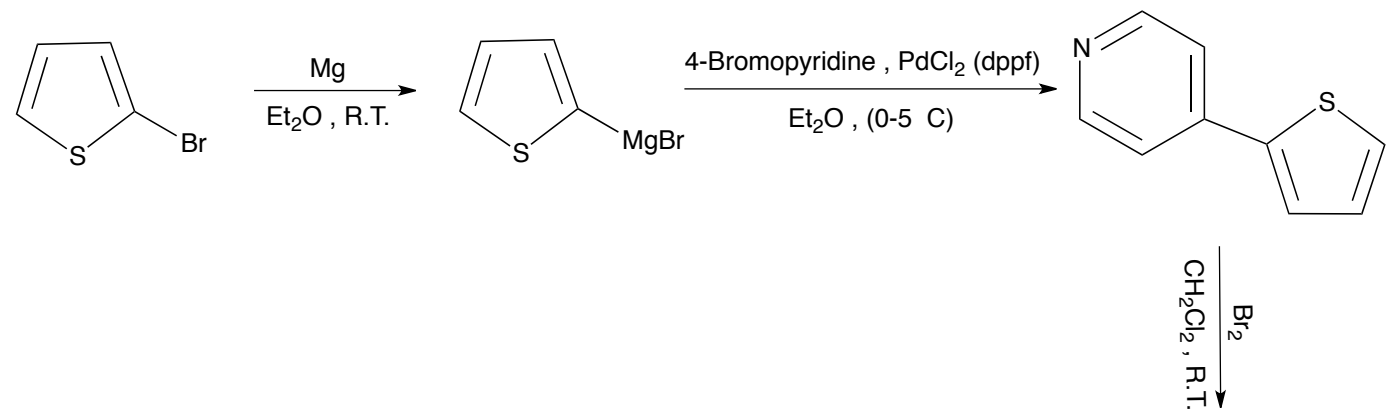

Malononitrile, $\mathrm{NaH}$, Catalyst<smiles>C=C(C)[C@@H]1C=CC(c2ccc(C(C#N)C#N)s2)=CC=[N+]1C</smiles>

Scheme 2.3. Synthesis of MPCT zwitterionic chromophore.

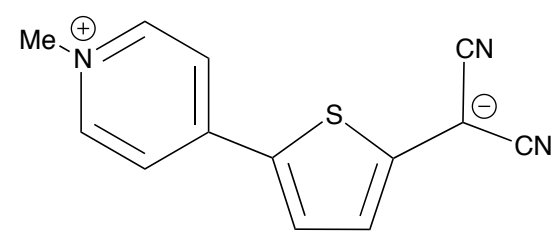

Zwitterionic Form (Ground State)

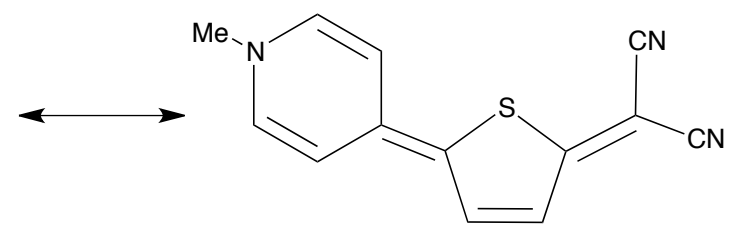

Neutral Form (Excited State)

Scheme 2.4. Resonance structures of MPCT chromophore. 


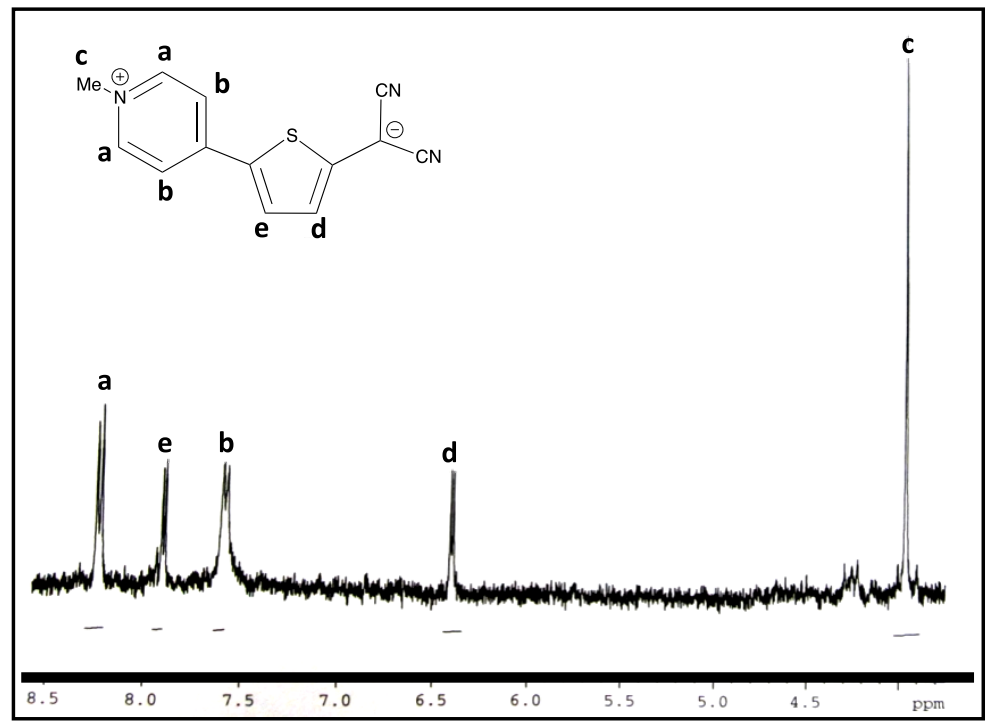

Figure 2.1. ${ }^{1} \mathrm{H}$ NMR spectrum $(300 \mathrm{MHz})$ of MPCT in DMSO- $d_{6}$.

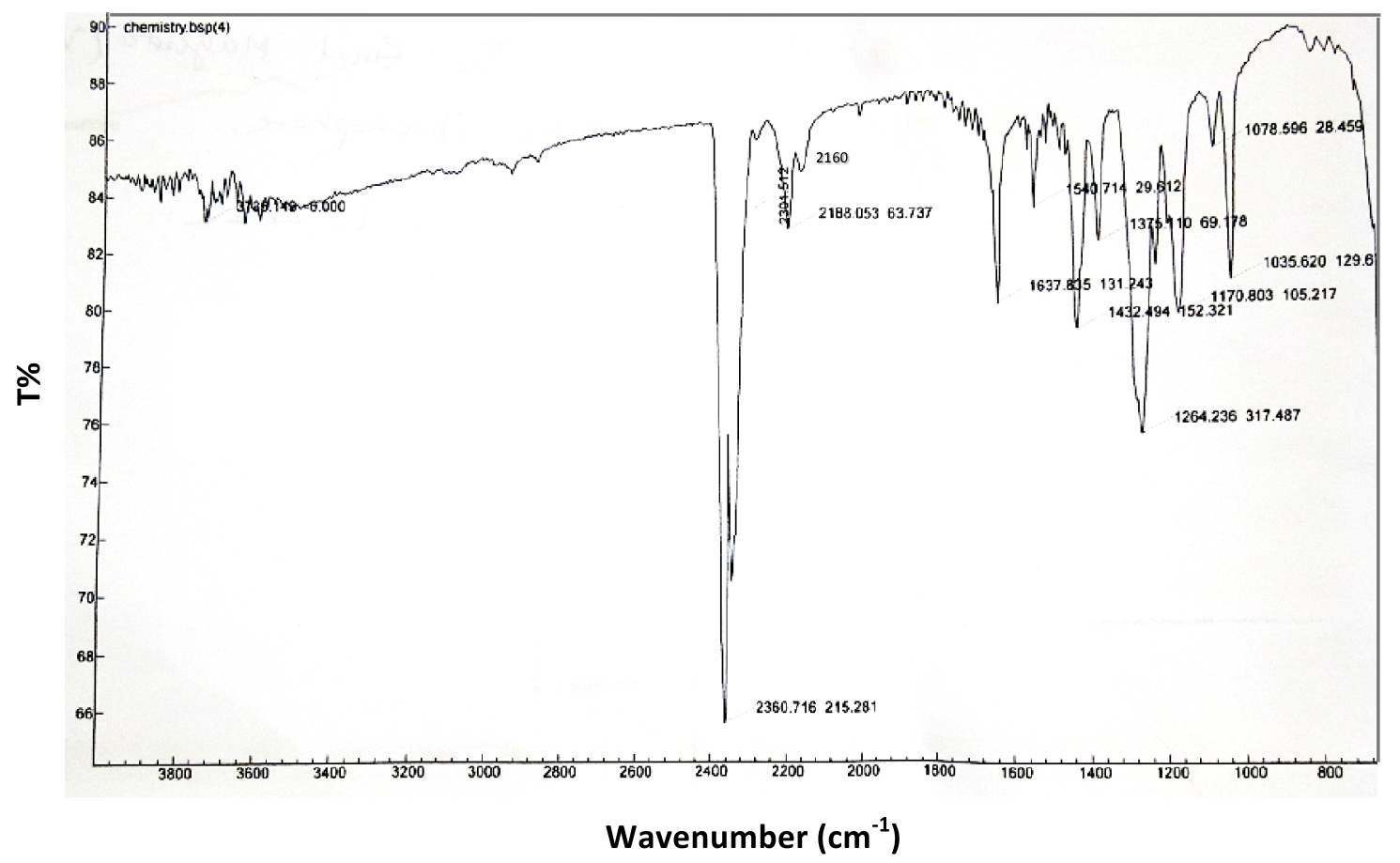

Figure 2.2. IR spectrum of MPCT (KBr Pellet). 


\subsubsection{Synthesis and Characterization of FDCN and PpQDM Chromophores}

FDCN is a zwitterionic chromophore with the negative charge on the dicyanomethanide group and the positive charge on the pyridinium's $\mathrm{N}$ atom and was formerly synthesized in our group using 4-fluorophenylacetonitrile and 4-pyridinecarboxaldehyde as starting materials (Scheme 2.5). The equilibrium between the zwitterion and quinoid states is shown in Scheme 2.6. In addition to the synthetic details of FDCN, characterizations by ${ }^{1} \mathrm{H}$ NMR, ${ }^{13} \mathrm{C}$ NMR, IR and mass spectrometry have been all mentioned in our previous work. ${ }^{9,11}$

PpQDM is another zwitterionic chromophore with the negative charge on the dicyanomethanide group and the positive charge on the pyridinium's $\mathrm{N}$ atom and was synthesized in our group previously by reaction of the mono-N-alkylated dipyridylium iodide with the lithium salt of TCNQ in the presence of a base (Scheme 2.7) and fully characterized. $^{10,11}$ 


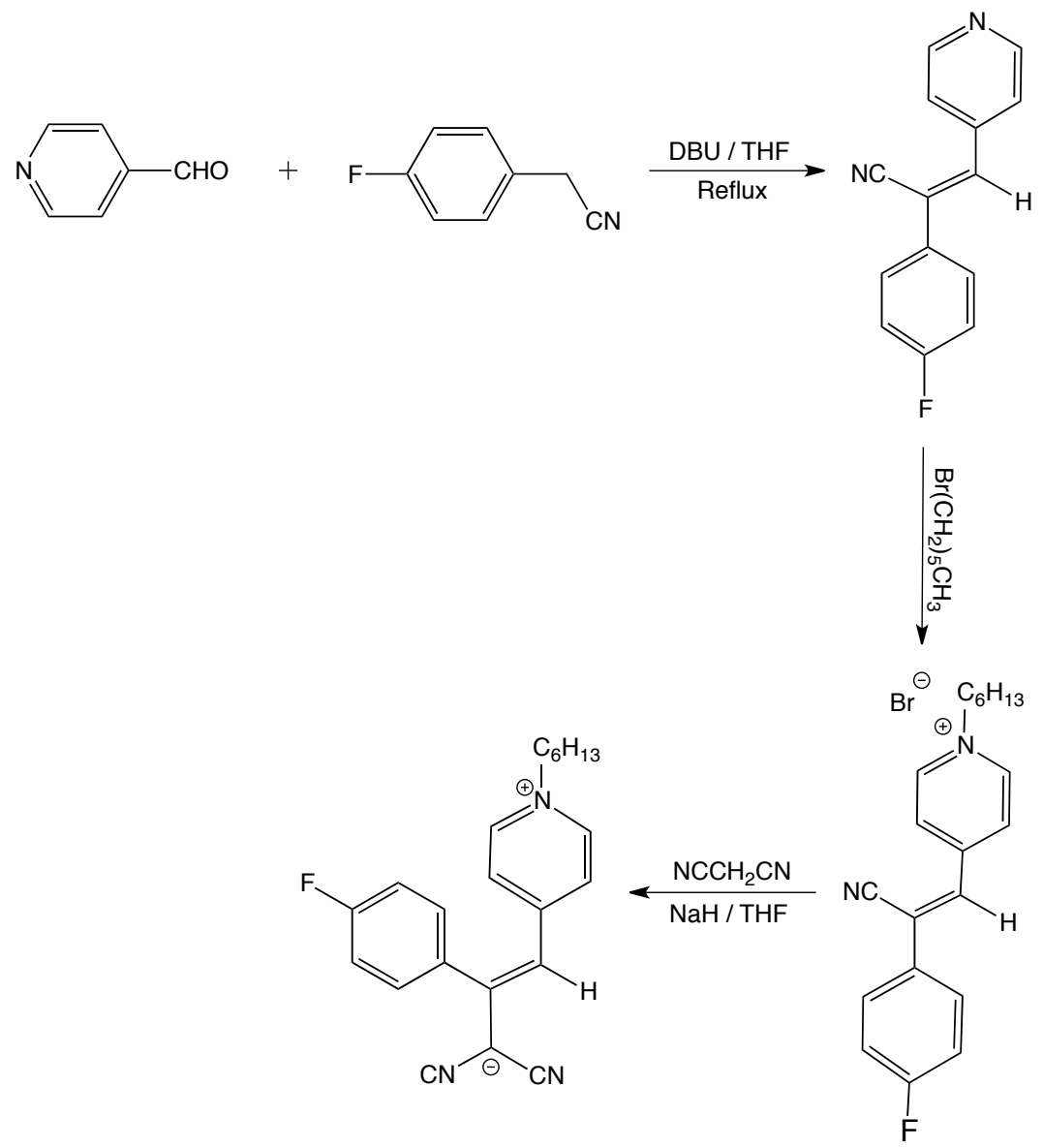

Scheme 2.5. Synthesis of FDCN zwitterionic chromophore.

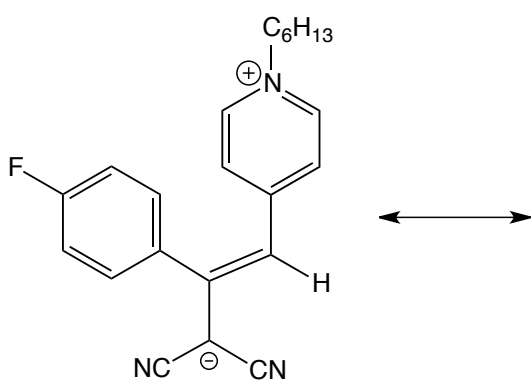

Zwitterionic Form (Ground State)<smiles>CCCCCCCN1C=CC(=CC(=C(C#N)C#N)c2ccc(F)cc2)C=C1</smiles>

Neutral Form (Excited State)

Scheme 2.6. Resonance structures of FDCN chromophore. 
<smiles>N#CC(C#N)c1ccc(C(C#N)C#N)cc1</smiles><smiles>Cc1ccnc(-c2cc(/C=C(\C#N)c3ccc(C(C#N)C#N)cc3)cc[n+]2C)c1</smiles>

Scheme 2.7. Synthesis of PpQDM zwitterionic chromophore.

FDCN and PpQDM are both solvatochromic in organic solvents, giving rise to a hypsochromic (or blue) shift in their absorption spectra by increasing the solvent polarity. In fact, this negative solvatochromism confirms that the zwitterionic form is favourable in zwitterion-quinoid equilibrium for the ground state (Scheme 2.8). ${ }^{10}$<smiles>Cc1ccnc(C2=C/C(=C/C3CCCCC3)C=CN2C)c1</smiles>

Scheme 2.8. Resonance structures of PpQDM chromophore. 


\subsubsection{UV-Vis Absorption}

The absorption spectra of MPCT, FDCN and PpQDM chromophores were measured in $\mathrm{MeCN}$ (Figure 2.3). All of the three chromophores exhibit intense absorption in the UVVis region. FDCN, MPCT and PpQDM have a maximal absorption at the wavelength $\left(\lambda_{\max }\right)$ of 484, 546, and $670 \mathrm{~nm}$ in MeCN, respectively. Molar extinction coefficients $(\varepsilon)$ of the three chromophores (Table 2.1) are relatively high, which can be attributed to CT from the negatively charge dicyanomethanide to the positively charged nitrogen in pyridinium. ${ }^{12,13}$

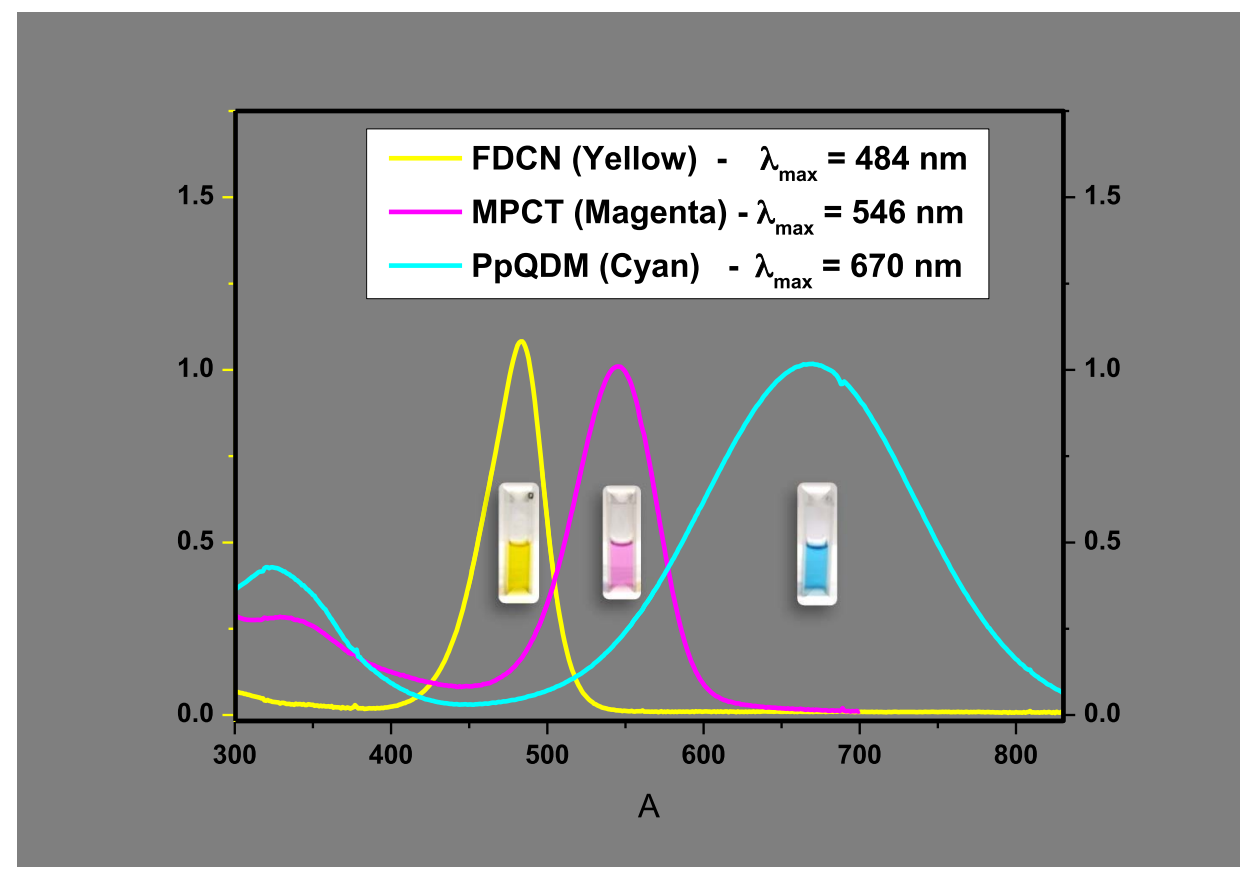

Figure 2.3. Optical absorption spectra of FDCN, MPCT, and PpQDM chromophores in $\mathrm{MeCN}$ solution. Inset: Photographs of the corresponding solutions.

Table 2.1. Molar extinction coefficients $(\varepsilon)$ of FDCN, MPCT and PpQDM in MeCN.

\begin{tabular}{|c|c|}
\hline Compound & $\boldsymbol{\varepsilon}\left(\mathbf{M}^{\mathbf{- 1}} \mathbf{c m}^{\mathbf{- 1}}\right)$ \\
\hline FDCN & 95,340 \\
\hline MPCT & 43,500 \\
\hline PpQDM & 27,000 \\
\hline
\end{tabular}




\subsubsection{Solvatochromic Properties of Zwitterionic Chromophores}

To investigate the solvatochromism of these zwitterionic chromophores, the maximal absorption peak positions were measured in various solvents. Table 2.2 collects absorption date relative to the solvatochromic behavior in the selected solvents.

Table 2.2. Optical absorption $\left(\lambda_{\max }, \mathrm{nm}\right)$ data for FDCN, MPCT and PpQDM.

\begin{tabular}{ccccccc}
\hline \multirow{2}{*}{ Compound } & \multicolumn{5}{c}{$\lambda_{\max }(\mathrm{nm})$} \\
\cline { 2 - 6 } & THF & Acetone & Methanol & DMF & MeCN \\
& $\mathbf{7 . 6 ^ { \mathrm { a } }}$ & $\mathbf{2 0 . 7}$ & $\mathbf{3 2 . 7}$ & $\mathbf{3 6 . 7}$ & $\mathbf{3 7 . 5}$ \\
\hline FDCN & 495 & 487 & 480 & 486 & 484 \\
MPCT & 586 & 556 & 537 & 545 & 546 \\
PpQDM & 810 & 713 & 647 & 680 & 670 \\
\hline
\end{tabular}

${ }^{\text {a }}$ Solvent Dielectric Constant $(\varepsilon)$ (Lide D. R. Handbook of Chemistry and Physics $76^{\text {th }}$ edition, P8-64, 1995-1996).

In this experiment, UV-Vis absorptions of the studied zwitterionic chromophores in these solvents showed a blue shift with increasing solvent polarity, in order of THF < Acetone $<$ Methanol $<\mathrm{DMF}<\mathrm{MeCN}$ (i.e., negative solvatochromism). For instance, the $\mathrm{CT}$ band of PpQDM is blue shifted from $810 \mathrm{~nm}$ in the less polar THF to $647 \mathrm{~nm}$ in the polar methanol, representing a shift of $\sim 163 \mathrm{~nm}$.

Consequently, greater stabilization of the zwitterionic form of the chromophore occurs in high polarity solvents rather than in low polarity ones, leading to higher energy absorption maximum in high polarity solvents. The same trend was observed in the case of 
FDCN chromophore, i.e., showing a blue shift in its absorption maxima in going from nonpolar to polar solvents.

Based on the solvatochromic study carried out on MPCT chromophore, strong CT band was observed in the visible region of the spectrum. It can be seen that by increasing the solvent polarity, the maximal absorption peak shifts form $586 \mathrm{~nm}$ in THF to $537 \mathrm{~nm}$ in $\mathrm{MeOH}$, or $49 \mathrm{~nm}$ shift towards the shorter wavelength.

As an example, Figure 2.4 shows the absorption spectra of MPCT in three different solvents. From the date and spectra recorded in different solvents of different polarity, it was determined that MPCT shows a strong negative solvatochromism with respect to its CT absorption band, namely, the position of the maximal absorption blue shifts with an increase of the solvent polarity.

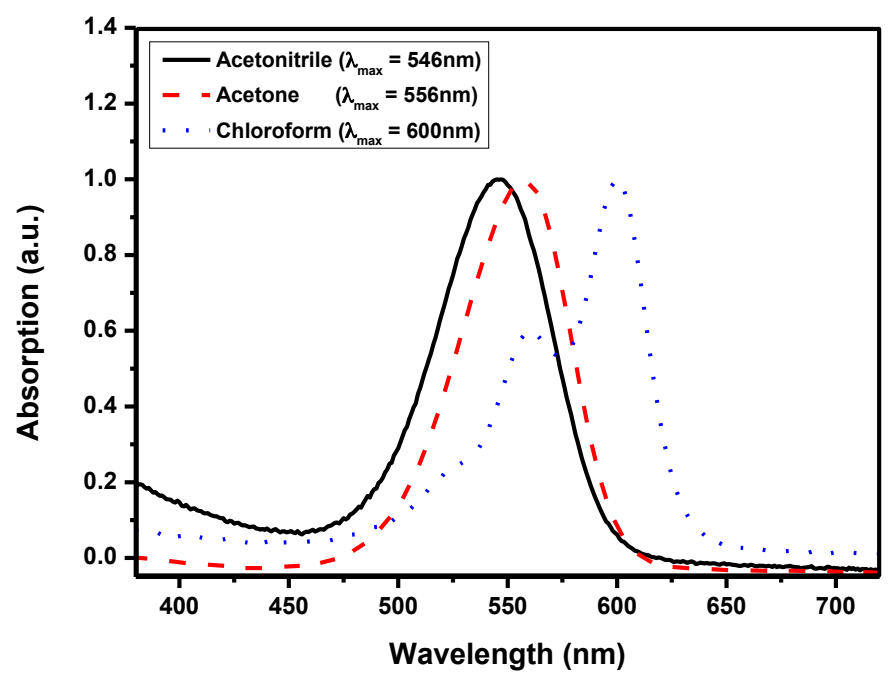

Figure 2.4. UV-Vis spectra of MPCT in acetonitrile, acetone and chloroform.

Furthermore, the magnitude of the highest solvatochromic shift for PpQDM $(\sim 163$ $\mathrm{nm})$ is significantly larger than the solvatochromic shift for FDCN $(\sim 15 \mathrm{~nm})$. This difference 
can be interpreted on the basis of the magnitude of the gain in resonance stabilization energy upon aromatization of the two donor and acceptor units. In other words, the gain in aromaticity for FDCN chromophore is expected to be less than in the case of PpQDM, attributing to the combination of a weaker acceptor and shorter conjugated bridge. Based on the solvatochromic shift for MPCT $(\sim 49 \mathrm{~nm})$, in spite of its larger degree of aromaticity comparing to FDCN due to the presence of thiophene in the conjugation system, the acceptor strength would be less than the one in the case of PpQDM chromophore leading to smaller solvatochromic shift than PpQDM.

\subsubsection{Halochromic Properties of Zwitterionic Chromophores in Solution}

PpQDM has a stronger CT band at $680 \mathrm{~nm}$ in DMF and gives a bright cyan color. In a previous test-tube experiment, ${ }^{11}$ upon addition of acid $(\mathrm{HCl})$ to PpQDM in DMF the cyan colored solution immediately turned to colorless and subsequent addition of DMF could bring the cyan color back. This experiment indicates halochromic behavior for PpQDM.

In fact, this reversible color-changing process is believed to be due to the protonation of the chromophore by acid and deprotonation by base (e.g., DMF) at the acceptor moiety. The mechanism for this reversible halochromism was proposed (Scheme 2.9). ${ }^{11}$ The protonated PpQDM was also prepared and characterized by IR in comparison with PpQDM. The spectral analyses indicated that the position and intensity of $v_{C \equiv N}$ at the acceptor moiety of PpQDM changed noticeably after protonation. Two lower frequency peaks of $v_{C \equiv N}$ shifted to a higher frequency, which is typical for the $\mathrm{C} \equiv \mathrm{N}$ group connected to the neutral saturated carbon atom. These results suggest that the negative charged part of the studied zwitterionic chromophores can function as a binding site to proton pertaining to the presence of the negative charge. 
<smiles>Cc1ccnc(-c2cc(/C=C(\C#N)c3ccc(C(C#N)(C#N)C#N)cc3)cc[n+]2C)c1</smiles>

PpQDM<smiles>Cc1ccnc(-c2cc(/C=C(\C#N)c3ccc(C(C#N)(C#N)C#N)cc3)cc[n+]2[O-])c1</smiles>

P-PpQDM

Scheme 2.9. Protonation and deprotonation of PpQDM.

Accordingly, the similar study was carried out for FDCN in this research work. The absorption of this chromophore in DMF is at $486 \mathrm{~nm}$ but shifts to a shorter wavelength at 476 $\mathrm{nm}\left(\Delta \lambda_{\max }=-10 \mathrm{~nm}\right)$ when the water was added to a volume ratio of 1:5 $\left(\mathrm{DMF} / \mathrm{H}_{2} \mathrm{O}\right)$. Thus, by adding an excess amount of $\mathrm{HCl}$ to the yellow DMF solution, the solution turned colorless immediately and the visible absorption band disappeared. In this case, subsequent addition of DMF to the acid-containing solution also brought back the original yellow color (Figure 2.5). 


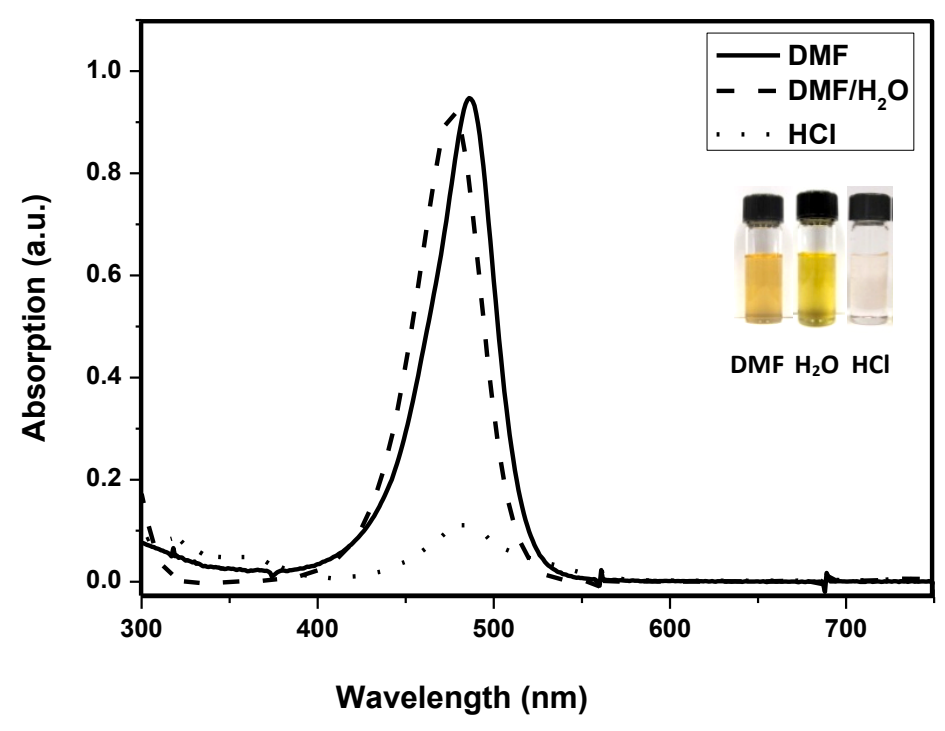

Figure 2.5. UV-Vis spectra of FDCN in DMF, $\mathrm{DMF} / \mathrm{H}_{2} \mathrm{O}$ and $\mathrm{HCl}$.

The study on MPCT revealed the same reversible protonation-deprotonation behavior with a negative halochromism $\left(\Delta \lambda_{\max }=-32 \mathrm{~nm}\right)$, which is between the values observed for FDCN and PpQDM (Figure 2.6).

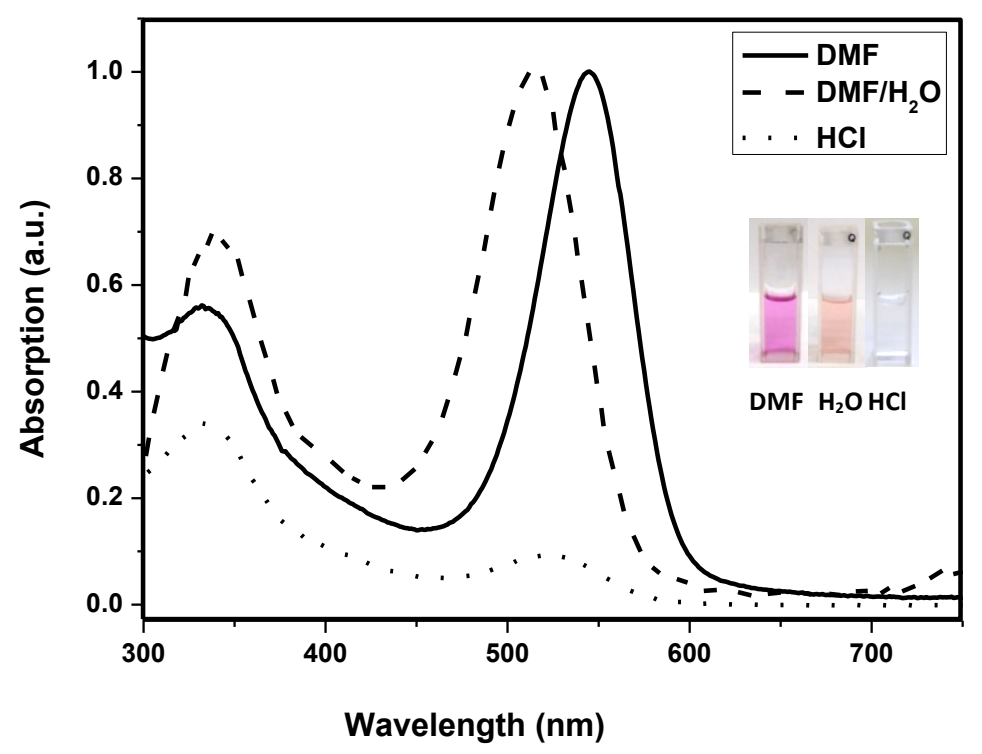

Figure 2.6. UV-Vis spectra of MPCT in DMF, $\mathrm{DMF} / \mathrm{H}_{2} \mathrm{O}$ and $\mathrm{HCl}$. 
Based on the reversible halochromism behavior investigated for all the three studied zwitterionic chromophores, it can be deduced that the negative charged part of the chromophores can function as a binding site to proton pertaining to the presence of the negative charge, whereas the chromophore can come back to the color state by deprotonation. The protonation-deprotonation process should be sensitive to the temperature and should also depend on the relative strength of the acid used. Therefore, another test was performed, employing a weaker acid and FDCN solution in chlorobenzene. Camphorsulfonic acid (CSA) is an organic acid with a $\mathrm{pK}_{\mathrm{a}}$ value of 1.2 and is weaker than $\mathrm{HCl}\left(\mathrm{pK}_{\mathrm{a}}=-7\right)$. FDCN was firstly treated with CAS, resulting in color bleaching or protonation of FDCN. In this case, addition of DMF to the protonated FDCN led to the formation of yellow-orange color (Figure 2.7). In a further experiment, the colorless solution turned to color upon heating the solution only. This thermo-halochromism behavior will be presented and discussed in detail in next chapter.

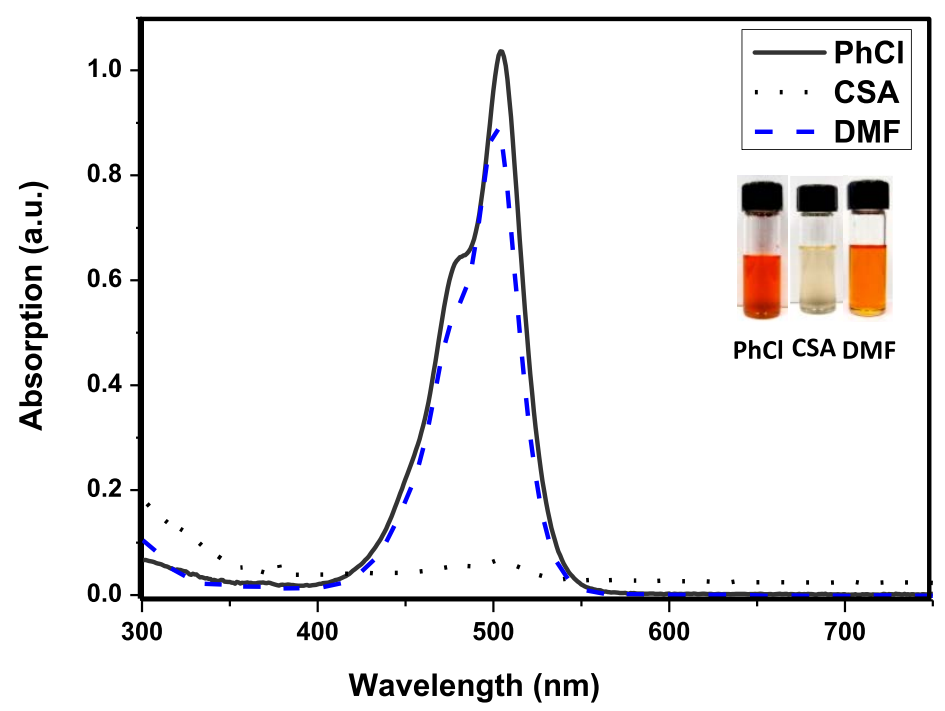

Figure 2.7. UV-Vis spectra of FDCN in $\mathrm{PhCl}$ with a mixture of CSA and DMF. 
The halochromic properties of the three zwitterionic chromophores were also investigated by monitoring the changes of absorption spectra in acetonitrile upon titration with a number of acids with different $\mathrm{pK}_{\mathrm{a}}$ values (Table 2.3).

Table 2.3. Halochromic properties of the zwitterionic chromophores in different acids.

\begin{tabular}{|l|c|c|}
\hline Acid & $\mathbf{p K}_{\mathbf{a}}$ Value & Bleaching Results \\
\hline Phenol & 9.99 & No Bleaching \\
Boric Acid & 9.24 & No Bleaching \\
$\mathrm{AlCl}_{3}$ (Aluminium Chloride) & 4.96 & No Bleaching \\
$\mathrm{AcOH}$ (Acetic Acid) & 4.76 & No Bleaching \\
6-Bromohexanoic Acid & 2.9 & Moderate Bleaching \\
$\mathrm{CSA}$ (Camphorsulfonic Acid) & 1.2 & Complete Bleaching \\
$\mathrm{TSA}$ (p-Toluenesulfonic Acid) & -2.8 & Complete Bleaching \\
$\mathrm{HCl}$ & -7.0 & Complete Bleaching \\
$\mathrm{H}_{2} \mathrm{SO}_{4}$ & -10 & Complete Bleaching \\
\hline
\end{tabular}

Based on the above results, it can be concluded that the halochromic behavior of the studied chromophores is greatly influenced by the acid strength. If the acid is too weak, the protonation does not proceed and the chromophore cannot be bleached. In fact, the dependency of the halochromic behavior of the zwitterionic chromophores on the dissociation constant values of the acids can also confirm the presence of charge transfer mechanism occurs as the result of the halochromic character of the chromophores. Accordingly, it is found that the $\mathrm{pK}_{\mathrm{a}}$ equal or less than 1.2 is a critical value for an acid to be capable of bleaching the solutions of these zwitterionic chromophores.

Moreover, all the bleached chromophores solutions could resume their original colors upon heating, only in the case of the use of relatively weak organic acids (i.e., CSA and TSA). No color was back in the solutions bleached by strong mineral protic acids (i.e., 
$\mathrm{HCl}$ and $\mathrm{H}_{2} \mathrm{SO}_{4}$ ). Therefore, CSA and TSA were chosen for further halochromic and thermohalochromic investigations (see Chapter 3).

\subsection{Conclusion}

Zwitterionic chromophore MPCT was synthesized and characterized by ${ }^{1} \mathrm{H}$ NMR and IR. All the three zwitterionic chromophores exhibited intense absorption at the maximum wavelength $\left(\lambda_{\max }\right)$ of 484,546 , and $670 \mathrm{~nm}$ in $\mathrm{MeCN}$ with the relatively high molar extinction coefficients, being attributed to the charge transfer from the negatively charge dicyanomethanide group to the positively charged nitrogen in pyridine. The negative solvatochromism with respect to the CT band depends on the conjugation length between the two charges of these chromophores, as indicated by the largest shift in absorption in order of FDCN $(-15 \mathrm{~nm})<\operatorname{MPCT}(-49 \mathrm{~nm})<\operatorname{PpQDM}(-163 \mathrm{~nm})$

The three zwitterionic chromophores are halochromic and the halochromism can proceed via the acid-base reaction, resulting in a significant color-to-colorless change or spectral shift in order of FDCN $\left(\Delta \lambda_{\max }=-10 \mathrm{~nm}\right)<\operatorname{MPCT}\left(\Delta \lambda_{\max }=-32 \mathrm{~nm}\right)<\operatorname{PpQDM}$ $\left(\Delta \lambda_{\max }=-100 \mathrm{~nm}\right)$. The halochromic behavior of the three zwitterionic chromophores depends on the dissociation constants of the employed acids. Therefore, the use of relatively weak acid allowed the thermally induced deprotonation or thermochromism of the protonated zwitterionic chromophores (Chapter 3).

\subsection{Experimental Section}

Materials. 4-Bromopyridine was obtained from the commercially available hydrochloride salt (TCI America) according to the literature procedure. ${ }^{21}$ Acetone was dried and distilled over $\mathrm{MgSO}_{4}$ under an atmosphere of dry nitrogen. 2-Bromothiophene, bromine, malononitrile, sodium hydride, methyl triflate, tetrakis(triphenylphosphine)palladium(0), 
magnesium, $\mathrm{PdCl}_{2}$, and solvents including anhydrous diethyl ether, methylene chloride, 1,2dimethoxyethane and toluene were all purchased from Aldrich Chemicals Canada and used as received.

General Methods. ${ }^{1} \mathrm{H}$ NMR spectra were recorded on a Varian $300 \mathrm{MHz}$ or a Bruker AMX $400 \mathrm{MHz}$ spectrometer using tetramethylsilane (TMS; $\delta=0 \mathrm{ppm}$ ) as an internal standard. The Fourier transform infrared (FTIR) spectra were recorded on a Perklin-Elmer1600 or a Bomen Michelson 120 FTIR spectrometer in the regions of $3600-600 \mathrm{~cm}^{-1}$. The melting points were determined using a Fisher-Johns melting point apparatus. The UV-Vis-NIR spectra were recorded on a Perklin-Elmer Lambda 900 UV-Vis-NIR spectrometer at room temperature. The solvatochromism study was conducted using chlorobenzene, THF, Acetone, Methanol, DMF, and MeCN. The absorption spectra of all samples were taken in a quartz cuvette with a path length of $10.0 \mathrm{~mm}$.

\section{Synthesis of MPCT Chromophore}

\section{4-(2-Thienyl)pyridine (1)}

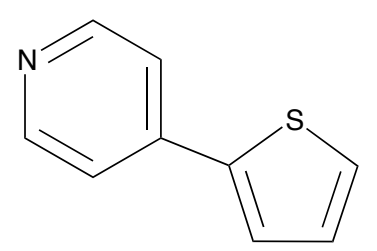

2-Thienylmagnesium bromide was prepared by adding a solution of 2bromothiophene $(4.93 \mathrm{~g}, 30.26 \mathrm{mmol})$ in anhydrous diethyl ether $(35 \mathrm{~mL})$ to a suspension of magnesium $(0.91 \mathrm{~g}, 37.41 \mathrm{mmol})$ in the same solvent $(10 \mathrm{~mL})$. Keeping this Grignard solution under dry nitrogen atmosphere, it was added dropwise to a stirred suspension of 4bromopyridine $(4.26 \mathrm{~g}, 26.96)$ and $\mathrm{PdCl}_{2}(\mathrm{dppf})(0.17 \mathrm{~g}, 0.23 \mathrm{mmol})$ in the same solvent $(15$ $\mathrm{mL}$ ) maintaining temperature between -30 and $-20{ }^{\circ} \mathrm{C}$. The immediately gained white 
precipitate was being stirred for $2 \mathrm{~h}$ at $0-5{ }^{\circ} \mathrm{C}$. Thereafter, the reaction mixture was poured into a saturated aqueous ammonium chloride solution $(60 \mathrm{~mL})$ to extract the aqueous layer with diethyl ether $(3 \times 20 \mathrm{~mL})$. Eventually, the practically pure compound (1) was collected as a light yellow solid after drying the combined organic layers and evaporating the solvent (3.04 g, 18.84 mmol, 70\%): mp: 88-91 ${ }^{\circ} \mathrm{C}\left(\right.$ lit. $^{28}$ 93-94 $\left.{ }^{\circ} \mathrm{C}\right) ;{ }^{1} \mathrm{H}$ NMR $\left(300 \mathrm{MHz}, \mathrm{CDCl}_{3}\right) \delta$ $8.59(\mathrm{~d}, 2 \mathrm{H}, J=4.8 \mathrm{~Hz}), 7.51(\mathrm{~d}, 1 \mathrm{H}, J=3.8 \mathrm{~Hz}), 7.49(\mathrm{~d}, 2 \mathrm{H}, J=4.8 \mathrm{~Hz}), 7.41(\mathrm{~d}, 1 \mathrm{H}, J$ $=5.0 \mathrm{~Hz}), 7.13(\mathrm{~d}, 1 \mathrm{H}, J=5.0,3.8 \mathrm{~Hz})$ represented in Figure S2.1.

\section{4-(5-Bromothien-2-yl)pyridine (2)}

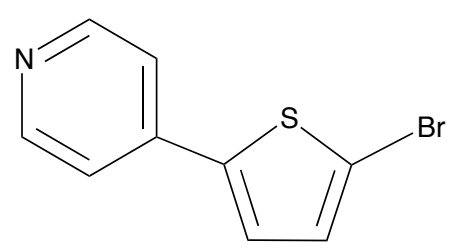

Bromine (5.99 g, $37.64 \mathrm{mmol})$ was added to a solution of 4-(2-thienyl)pyridine (1) (3.04 g, $18.83 \mathrm{mmol})$ in methylene chloride $(130 \mathrm{~mL})$. After being stirred for $15 \mathrm{~min}$ at room temperature, the reaction mixture was poured onto $10 \%$ aqueous $\mathrm{K}_{2} \mathrm{CO}_{3}(130 \mathrm{~mL})$, and methylene chloride was employed to extract the aqueous layer $(3 \times 100 \mathrm{~mL})$. Then, the combined organic layers were dried and the solvent was evaporated to give a brown solid (2.04 g, $8.50 \mathrm{mmol}, 67 \%): \mathrm{mp} 140-145{ }^{\circ} \mathrm{C}\left(\right.$ lit. $\left.^{21} 152-153^{\circ} \mathrm{C}\right) ;{ }^{1} \mathrm{H} \mathrm{NMR}\left(300 \mathrm{MHz}, \mathrm{CDCl}_{3}\right) \delta$ $8.57(\mathrm{~d}, 2 \mathrm{H}, J=6.1 \mathrm{~Hz}), 7.36(\mathrm{~d}, 2 \mathrm{H}, J=6 \mathrm{~Hz}), 7.24(\mathrm{~d}, 1 \mathrm{H}, J=4.0 \mathrm{~Hz}), 7.08(\mathrm{~d}, 1 \mathrm{H}, J=4$ $\mathrm{Hz}$ ) represented in Figure S2.2. 


\section{4-[5-(Dicyanomethanido)thien-2-yl]pyridine Sodium Salt (3)}<smiles>N#CC(C#N)c1ccc(-c2ccncc2)s1</smiles>

A mixture of malononitrile $(1.36 \mathrm{~g}, 20.72 \mathrm{mmol})$, which has been added in portions to an ice-cooled suspension of sodium hydride (1.66 g, 60\% in oil, $41.42 \mathrm{mmol})$ in 1,2dimethoxyethane $(70 \mathrm{~mL})$, was stirred at room temperature for 20 min under nitrogen. 4-(5Bromothien-2-yl)pyridine (2) (2.04 g, $8.49 \mathrm{mmol})$ and tetrakis(triphenylphosphine)palladium(0) (0.99 $\mathrm{g}, 0.86 \mathrm{mmol})$ were added to the above solution, and consequently the mixture was heated under reflux for $2 \mathrm{~h}$. The resulting precipitate was collected by filtration, and to eliminate the catalyst it was washed with toluene $(10 \mathrm{~mL})$, and then recrystallized from $\mathrm{H}_{2} \mathrm{O}$ to give the product as a yellow solid $(0.82 \mathrm{~g}, 2.97 \mathrm{mmol}, 40 \%): \mathrm{mp}>240{ }^{\circ} \mathrm{C} ;{ }^{1} \mathrm{H} \mathrm{NMR}$ $\left(300 \mathrm{MHz}, \mathrm{DMSO}-\mathrm{d}_{6}\right) \delta 8.31(\mathrm{~d}, 2 \mathrm{H}, \mathrm{J}=6.3 \mathrm{~Hz}), 7.43(\mathrm{~d}, 1 \mathrm{H}, \mathrm{J}=3.1 \mathrm{~Hz}), 7.27(\mathrm{~d}, 2 \mathrm{H}, \mathrm{J}=$ $6.3 \mathrm{~Hz}), 6.11(\mathrm{~d}, 1 \mathrm{H}, \mathrm{J}=3 \mathrm{~Hz})$ represented in Figure S2.3.

\section{2-(4-N-Methylpyridinium)-5-(dicyanomethanido)-thiophene (MPCT) (4)}

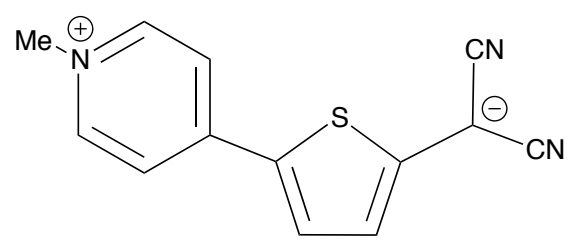

A suspension of 4-[5-(dicyanomethanido)thien-2-yl]pyridine sodium salt (3) (0.82 g, $3.32 \mathrm{mmol})$ was prepared in dry acetone $(50 \mathrm{~mL})$ and methyl triflate $(0.81 \mathrm{~g}, 0.54 \mathrm{~mL}, 4.97$ mmol) was added dropwise. The color of the mixture immediately changed from yellow to 
violet. The precipitate was collected after it was being stirred overnight at room temperature.

Thereafter, the precipitate was washed with water and then with $\mathrm{EtOH}$ to give the analytically pure product $(0.61 \mathrm{~g}, 2.55 \mathrm{mmol}, 74 \%)$ as a violet solid: $\mathrm{mp}>240{ }^{\circ} \mathrm{C} ;{ }^{1} \mathrm{H} \mathrm{NMR}(300 \mathrm{MHz}$, DMSO- $\left.d_{6}\right) \delta 8.26(\mathrm{~d}, 2 \mathrm{H}, J=7.0 \mathrm{~Hz}), 7.92(\mathrm{~d}, 1 \mathrm{H}, J=4.4 \mathrm{~Hz}), 7.62(\mathrm{~d}, 2 \mathrm{H}, J=7.0 \mathrm{~Hz})$, $6.41(\mathrm{~d}, 1 \mathrm{H}, J=4.4 \mathrm{~Hz}), 3.96(\mathrm{~s}, 3 \mathrm{H} \mathrm{Hz})$ represented in Figure 2.1. 


\subsection{References}

(1) (a) Rao, V. P.; Jen, A. K.-Y.; Wong, K. Y.; Drost, K. J. Tetrahedron Lett. 1993, 34, 1747. (b) Wong, K. Y.; Jen, A. K.-Y.; Rao, V. P.; Drost, K. J. J. Chem. Phys. 1994, 100,6818 .

(2) (a) Gilmour, S.; Marder, S. R.; Perry, J. W.; Cheng, L.-T. Adv. Mater. 1994, 6, 494.

(b) Bedworth, P. V.; Cai, Y.; Jen, A.; Marder, S. R. J. Org. Chem. 1996, 61, 2242.

(3) Alcade, E. Adv. Heterocycl. Chem. 1994, 60, 197.

(4) (a) Paley, M. S.; Harris, J. M. J. Org. Chem. 1991, 56, 568.

(5) (a) Dalton, L. R.; Harper, A. W.; Ghosn, R.; Steier, W. H.; Ziari, M.; Fetterman, H.; Shi, Y.; Mustacich, R. V.; Jen, A. K.- Y.; Shea, K. J. Chem. Mater. 1995, 7, 1060. (b) Drost, K. J.; Jen, A. K.-Y.; Rao, V. P. Chemtech. 1995, 25, 16.

(6) (a) Bradamante, S.; Pagani, G. A. Pure Appl. Chem. 1989, 61, 709. (c) Barchiesi, E.; Bradamante, S.; Ferraccioli, R.; Pagani, G. A. J. Chem. Soc., Perkin Trans.2 1990, 375.

(7) (a) Blenkle, M.; Boldt, P.; Brauchle, C.; Grahn, W.; Ledoux, I.; Nerenz, H.; Stadler, S.; Wichern, J.; Zyss, J. J. Chem. Soc., Perkin Trans. 1996, 2, 1377. (b) Alain, V.; Fort, A.; Barzoukas, M.; Chen, C.-T.; Blanchard-Desce, M.; Marder, S. R.; Perry, J. W. Inorg. Chim. Acta 1996, 242, 43.

(8) Abbotto, A.; Bradamante S.; Facchetti, A.; Pagani, G. A. J. Org. Chem. 1997, 62, 5755.

(9) Hao, W. H.; Wang, C.; Qian, G.; Wang, Z. Y. Acta Cryst. 2012, E68, o82.

(10) Hao, W. H.; McBride, A.; McBride, S.; Gao, J. P.; Wang, Z. Y. J. Mater Chem. 2011, 21,1040 .

(11) Hao, W. H. "Development of Zwitterionic Chromophores and Nonlinear Optical Polymers for Electro-Optic and Near Infrared Chemosensors Applications", Ph. D. Thesis, Carleton University 2011.

(12) Beaudin, A.; Song, N. H.; Bai, Y. W.; Men, L. Q.; Gao, J. P.; Wang, Z. Y.; Szablewski, M.; Cross, M.; Wenseleers, W.; Campo J.; Goovaerts, E. J. Chem. Mater. 2006, 18, 1079. 
(13) Staneva, D.; Grabchev, I.; Soumillion, J. P.; Bojinov, V. J. Photochem. Photobio. 2007, 189, 192. 


\section{Chapter 3 Thermo-halochromic Properties of Zwitterionic Chromophores}

\subsection{Introduction}

Thermochromic (TC) materials, due to their temperature-sensitive features, are widely explored as "smart materials" for use in devices such as tuneable light filters, thermal indicators, chemo/biosensors, smart windows, imaging devices and optical switching. In this regard, organic thermochromic compounds have become increasingly important in recent years, both in academic studies and industrial applications such as textile and smart coatings. ${ }^{1-4}$ Particularly, organic materials consisting of a leuco dye (electron donor), a weak acid (electron acceptor; known as the developer) and a solvent were initially investigated. .- $^{5-}$ ${ }^{10}$ In the past two decades, many of TC materials based on Crystal Violet Lactone (CVL) have been reported as the three-component thermochromic materials. ${ }^{11,12}$ Furthermore, halochromic substances that are suitable to be used in environments where $\mathrm{pH}$ changes occur frequently, or places where alternations in $\mathrm{pH}$ are extreme, can behave as indicators to determine the $\mathrm{pH}$ of solutions by observing the color changes once the indicator is mixed with the unknown solutions. ${ }^{13-15}$

Thermochromic polymers based on leuco dye-developer-solvent systems have been described in literatures. ${ }^{15}$ Polyethylene was initially tried as a polymer matrix. In this regard, adding the both developer and the leuco dye would lead to a blue color in the solid state and colorless in the molten state. The same behavior has been observed substituting polyethylene with polypropylene. It was observed that thermochromic switching mechanism in such thermochromic polymer materials is the same as in leuco dye-developer-solvent systems. Moreover, investigations on the concentration dependence of the thermochromic composites (leuco dye, developer and solvent) of thermoplastic polyolefins revealed a direct 
proportionality between the composite concentration and resulting color intensity according to the Beer's Law. ${ }^{16}$

In general, the thermochromic material composition can comprise plastics, films, papers, fibers, and solvents, coatings, and ink materials. Accordingly, the research and development of organic thermochromic systems are focused on the following concepts: 1) improvements of thermal stability of thermochromic chromophores for doping in polymer matrices, 2) expansion of the switching temperature range of thermochromic chromophores, 3) significant increase of the UV-visible-near infrared light stability of leuco dye-developersolvent systems, 4) inverse thermochromic switching from a colorless to a colored state by heating, and 5) comprehension of thermochromic materials in displays, devices, modulus or electro-optical equipments of any kind. The reported examples with such thermochromic switching behavior have been of academic interests since two decades ago, ${ }^{17-19}$ For instance, the use of thermochromic materials for multiple temperature-indicating sensors comprising reversible and irriversible thermochromic switching chromophores or thermochromic hybrid organic-inorganic structures have been documented. ${ }^{15}$

In this regard, one of research tasks as described in this chapter involves the investigation of the thermo-halochromic properties of FDCN, MPCT and PpQDM chromophores as a guest in three different media: solution, gel network and polymer.

Given that these chromophores are thermo-halochromic or can change their absorption profile with changes in temperatures and $\mathrm{pH}$, it would be possible to use them as a switch to trigger the change of another fluorescent dye. By doing so, one can formulate a range of halofluorescent and thermofluorescent dyes from some simple fluorescent dyes and 
these zwitteronic chromophores. Fluorescent dyes are often used as a probe or tag for sensing and visualizing the analytes in biological and environmental applications. ${ }^{20-23}$

In order to achieve an efficient energy transfer, the fluorescent dyes should be selected to have the emission wavelengths matching well with the absorption wavelength of these chromophores in either zwitterionic form or protonated form. Among the numerous classes of fluorescent dyes, perylene dyes are unique due to their considerable photophysical properties in addition to their high thermal and photophysical stabilities. Perylene, as the fundamental compound of perylene family, emits strongly around $450 \mathrm{~nm} .^{24,25}$

Rhodamine dyes, which are based on xanthene scaffolds, are known as one of the highly efficient fluorescent dyes. Rhodamines have been considerably attracted interest from chemists pertaining to their excellent photophysical properties. Accordingly, Rhodamine dyes have been widely used as platforms to design fluorescent probes due to their desirable photophysical properties including high molar extinction coefficients and high quantum yields. Rhodamine $6 \mathrm{G}$ is known as one of the highly fluorescent dyes in the Rhodamine family with strong emission around $550 \mathrm{~nm}$ used as a tracer dye to determine the rate and direct of the water flow and transport. ${ }^{26}$

Accordingly, another task as described in this chapter is to study a reversible fluorescence turn-on/off behavior in a system including perylene and Rhodamine 6G with FDCN. Herein, FDCN is selected as fluorescent quencher, owing to its suitable absorption profile $\left(\lambda_{\max }=507 \mathrm{~nm}\right.$ in $\left.\mathrm{PhCl}\right)$ and thermo-halochromic property. 


\subsection{Results and Discussion}

\subsubsection{Thermo-halochromic Properties of Zwitterionic Chromophores in Solution}

To evaluate the thermo-halochromic properties of the three zwitterionic chromophores in solution, firstly the changes of absorption spectra of the chromophores were recorded by titration with the two appropriate acids mentioned in the previous chapter (i.e., TSA and CSA). The results show that the $\mathrm{pK}_{\mathrm{a}}$ values of the employed acids affect the bleaching ratios (Figure 3.1); TSA $\left(\mathrm{pK}_{\mathrm{a}}=-2.8\right)$ can bleach each of the chromophores faster than CSA $\left(\mathrm{pK}_{\mathrm{a}}=1.2\right)$. Of course, pertaining to a further test carried out on the bleached samples, color of the solutions was observed to be back easier, faster and more significant in the case of using CSA relative to TSA. Therefore, CSA was eventually a candidate as the most appropriate acid to efficiently provide the both bleaching and reversible thermohalochromic switching of the zwitterionic chromophores.

By comparing the results of acid titration of the three zwitterionic chromophores, it is clear that the proton affinity increases in the conjugated system with an increase of the $\pi$ conjugation length from FDCN to PpQDM, leading to the use of a smaller amount of acid for bleaching the same concentration of the chromophore. The conjugation length of these zwitterionic chromophores strongly affects the charge transfer (CT), which also causes an overall bathochromic shift (i.e., changing color from yellow to cyan via switching from a 6 carbon-carbon double bond conjugation system in the case of FDCN to a 10 carbon-carbon one in the case of PpQDM). ${ }^{27}$ 

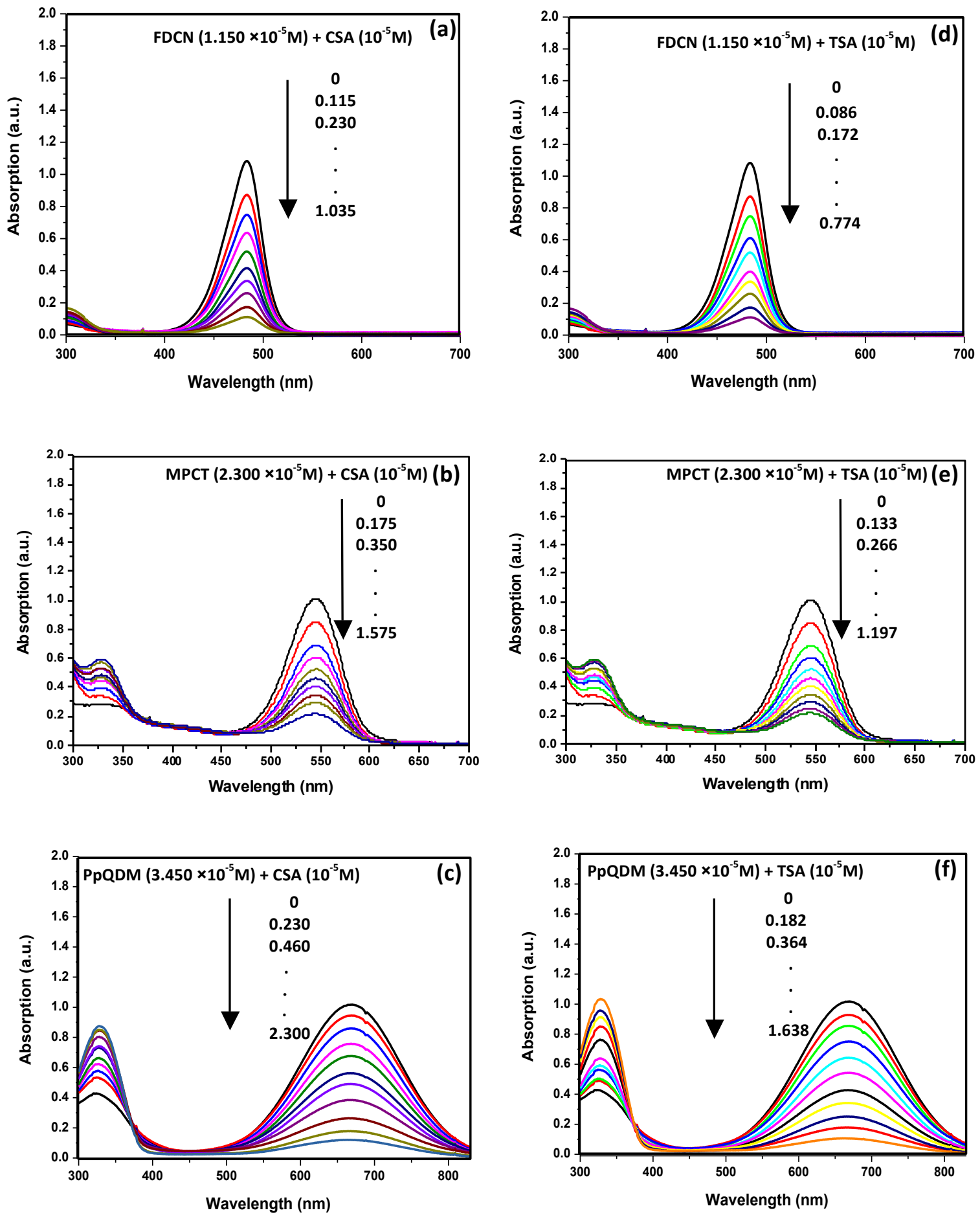

Figure 3.1. Changes in absorption spectra of FDCN, MPCT, and PpQDM in acetonitrile $\left(\sim 10^{-5} \mathrm{M}\right)$ with CSA (a, b, and c) and TSA (d, e, and f). 
In particular, the different titration rates observed for the three chromophores imply that the negatively charged $\mathrm{C}(\mathrm{CN})_{2}$ moiety has a higher affinity to the acid proton in the case of PpQDM comparing to the other two chromophores. Based on the previous discussion on the structure-property relationship of the three chromophores, this can be attributed to greater magnitude of the aromaticity and the resonance stabilization energy regarding the combination of a stronger acceptor moiety and longer conjugated bridge. In the case of MPCT, in spite of its larger degree of aromaticity than FDCN due to the presence of thiophene in the conjugation system, the acceptor strength would be less than the one in the case of PpQDM chromophore.

The mixture of FDCN and PpQDM was further employed in acetonitrile with two different concentration ratios of the two chromophores (i.e., 1:1 and 1:3), using CSA and TSA to perform the acid titration. As shown in Figure 3.2, in both ratios PpQDM is competitively bleached faster than FDCN and the bleaching occurs faster with TSA. In other words, protonation of zwitterionic chromophore can be tuned by changing the strength of the acceptor due to the mutual influence of the degree of aromaticity and conjugation. 

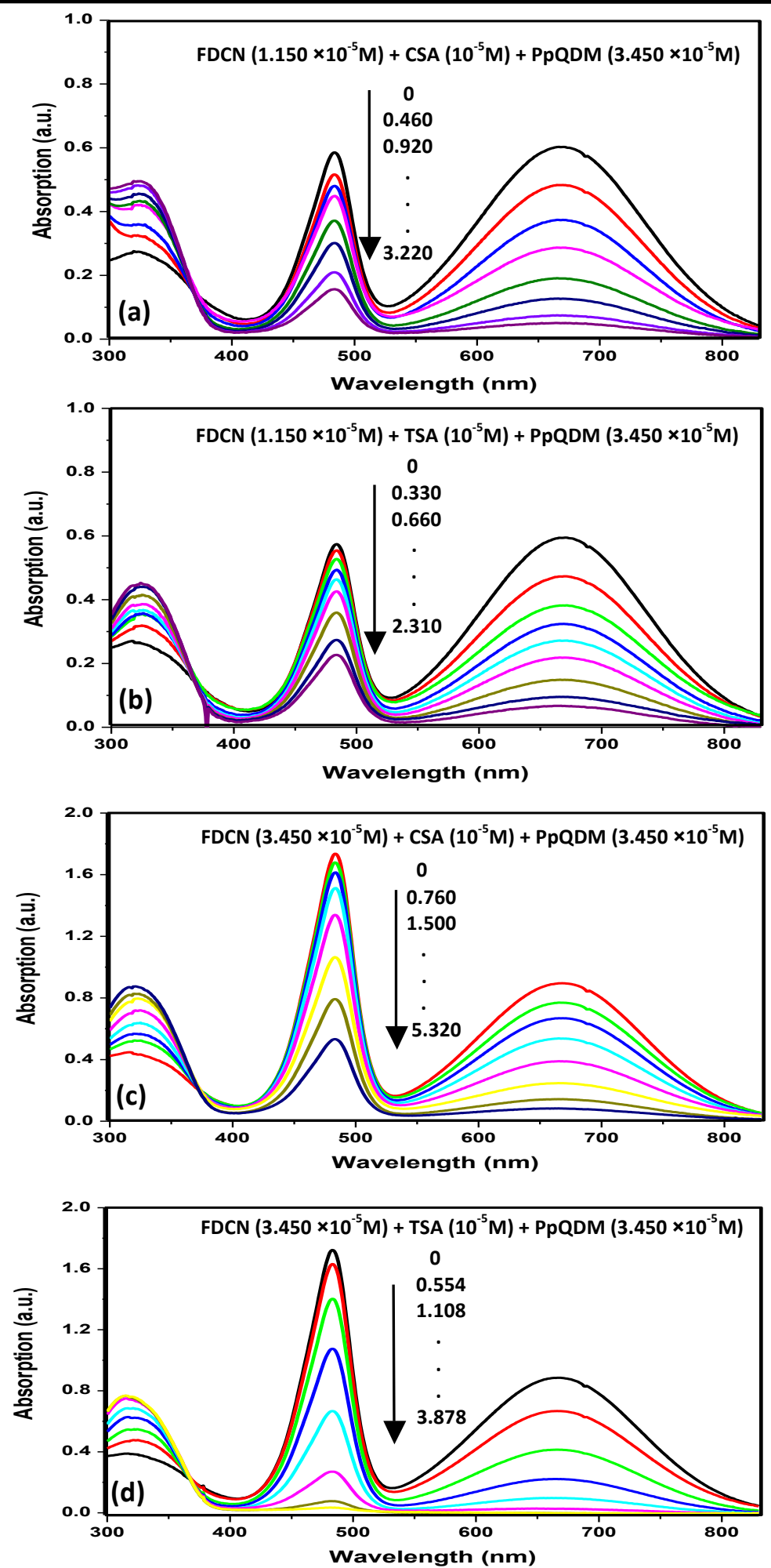

Figure 3.2. Changes in absorption spectra of FDCN in acetonitrile $\left(\sim 10^{-5} \mathrm{M}\right)$ as a mixture with (a, b) 3 equiv. of PpQDM $\left(\sim 10^{-5} \mathrm{M}\right)$ and (c, d) 1 equiv. of $\operatorname{PpQDM}\left(\sim 10^{-5} \mathrm{M}\right)$ with a different amount of CSA and TSA. 
Thermochromic behavior was then evaluated by heating the FDCN-H ${ }^{+}$and PpQDM$\mathrm{H}^{+}$solutions in acetonitrile at 40,60 and $80{ }^{\circ} \mathrm{C}$. Each of the trials was repeated for three cycles, in which all the samples showed reversible thermo-halochromic behavior (Figures 3.3 and 3.4). The higher the heating temperature is, the more the color of the bleached solution could return. In other words, the deprotonation of $\mathrm{FDCN}-\mathrm{H}^{+}$and $\mathrm{PpQDM}-\mathrm{H}^{+}$is accelerated at elevated temperatures. Upon cooling to the ambient temperature, the absorption intensity at the maximum wavelength of each chromophore decreased, representing a reversible thermo-halochromic behavior. It can be deduced that a temperature-dependent equilibrium exists between the primary bleached state and the colored one, which is believed to be due to the protonation of the chromophore at the $\mathrm{C}(\mathrm{CN})_{2}$ moiety by acid and consequently deprotonation as a result of heating (Scheme 3.1). The experimental observations showed noticeable color return in the case of FDCN. In comparison, a greater proton affinity in PpQDM with a stronger acceptor group requires a higher energy to dissociate the hydrogen bond, in order to return it to its colored zwitterionic state. 


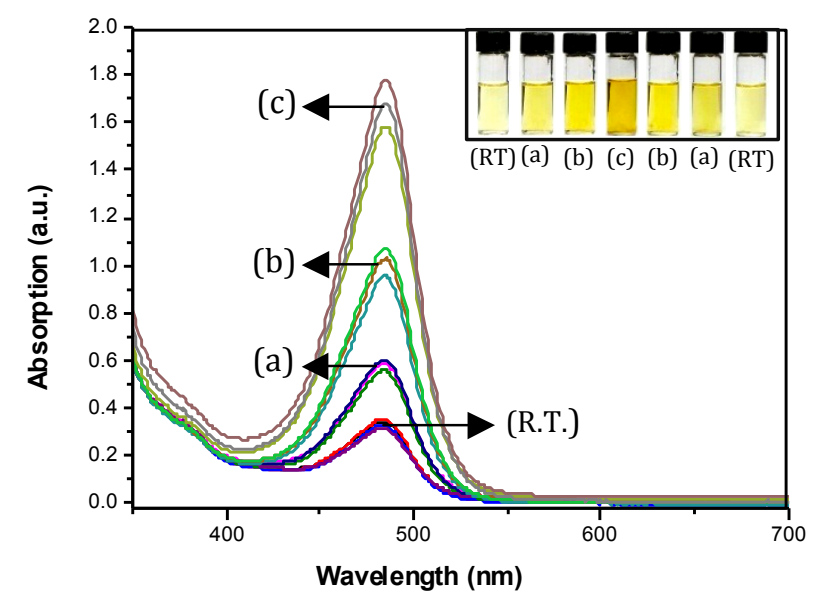

Figure 3.3. Changes in absorption spectra of $\mathrm{FDCN}_{-} \mathrm{H}^{+}$in acetonitrile $\left(\sim 10^{-5} \mathrm{M}\right)$ upon heating from room temperature to (a) $40{ }^{\circ} \mathrm{C}$, (b) $60{ }^{\circ} \mathrm{C}$, (c) $80{ }^{\circ} \mathrm{C}$ (three successive cycles). Inset: Photographs of the corresponding FDCN solution in acetonitrile at different temperatures.

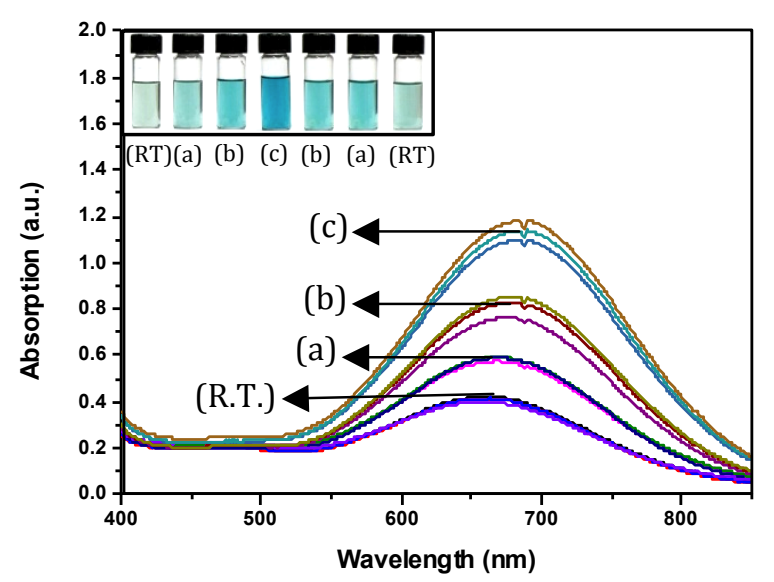

Figure 3.4. Changes in absorption spectra of $\mathrm{PpQDM}-\mathrm{H}^{+}$in acetonitrile $\left(\sim 10^{-5} \mathrm{M}\right)$ upon heating from room temperature to (a) $40{ }^{\circ} \mathrm{C}$, (b) $60{ }^{\circ} \mathrm{C}$, (c) $80{ }^{\circ} \mathrm{C}$ (three successive cycles). Inset: Photographs of the corresponding $\mathrm{PpQDM}$ solution in acetonitrile at different temperatures. 


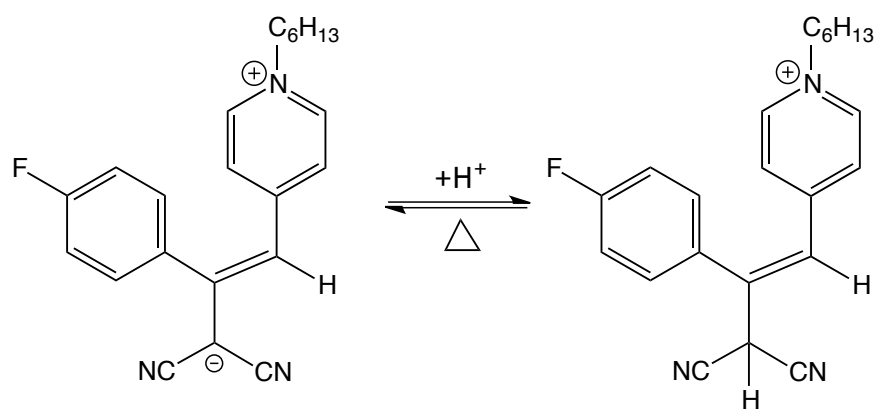

Scheme 3.1. Schematic presentation of protonation-deprotonation process of FDCN.

\subsubsection{Thermo-halochromic Properties of Zwitterionic Chromophores in}

\section{Malononitrile}

Malononitrile (dicyanomethane), known as a cyanocarbon compound, has been widely used as a reactant in a number of organic syntheses in the realm of medical, industrial and agricultural chemistry for more than 50 years. ${ }^{28}$ In this research, it was employed as a solvent to study the thermo-halochromic behavior of the three zwitterionic chromophores. The results are presented in Figure 3.5 to Figure 3.7.

Based on the results and observations, the same tendency was found in the bleaching rate of the three zwitterionic chromophores. PpQDM with the strongest acceptor moiety and the highest proton affinity was bleached faster than MPCT and FDCN. However, in all three cases, the results showed a higher demand for acid relative to those done in acetonitrile as the solvent. The nucleophilic character of the dicyanomethine moiety of malononitrile can act as a source of attracting proton, which may explain why this system needs more acid to be used. $^{28}$ 


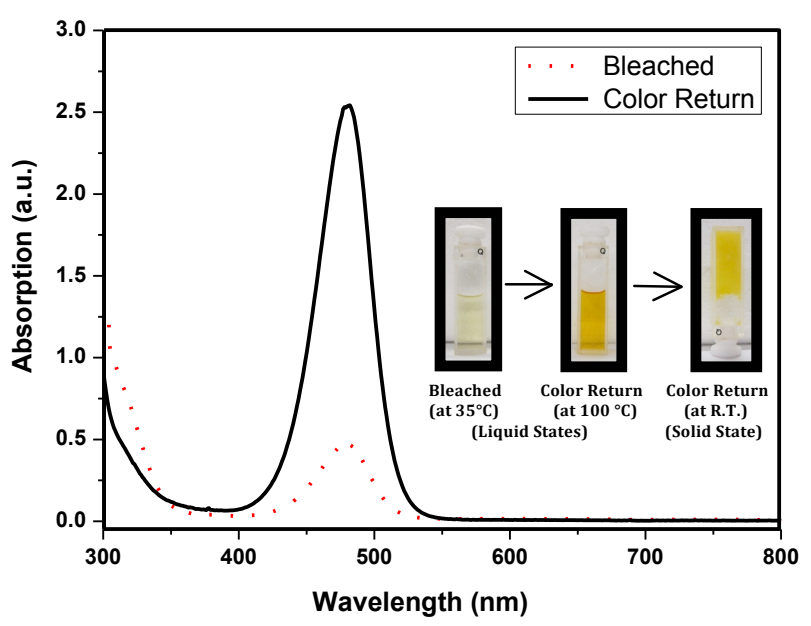

Figure 3.5. Irreversible thermo-halochromic behavior of FDCN in malononitrile. Inset: Photographs of the corresponding FDCN solution in malononitrile at three different sates, i.e., liquid state of the bleached (at $35^{\circ} \mathrm{C}$ ) and color return (at $100^{\circ} \mathrm{C}$ ) samples, and solid state of the color return sample (at R.T.).

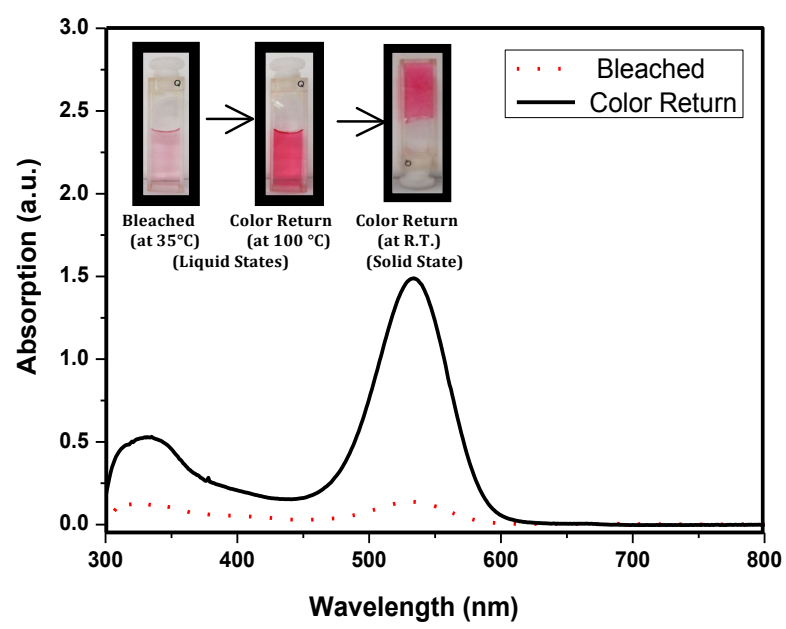

Figure 3.6. Irreversible thermo-halochromic behavior of MPCT in malononitrile. Inset: Photographs of the corresponding MPCT solution in malononitrile at three different sates, i.e., liquid state of the bleached (at $35^{\circ} \mathrm{C}$ ) and color return (at $100^{\circ} \mathrm{C}$ ) samples, and solid state of the color return sample (at R.T.). 


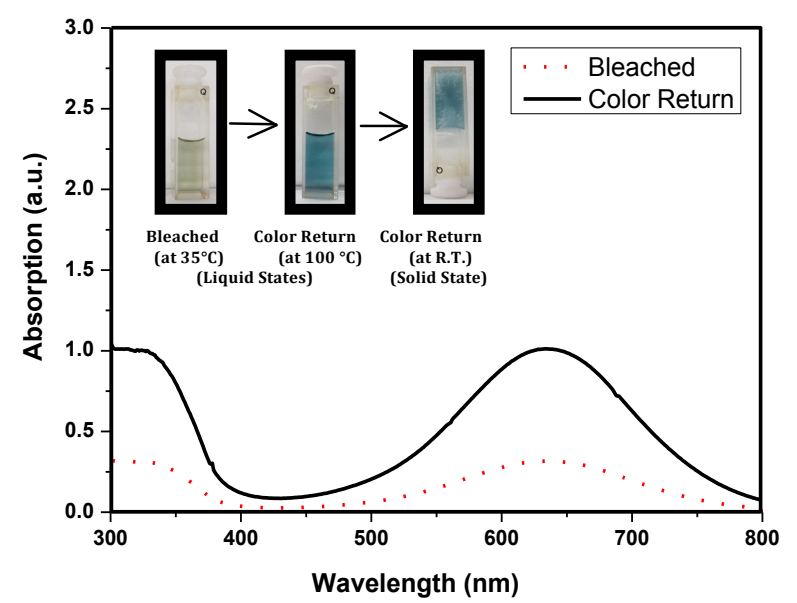

Figure 3.7. Irreversible thermo-halochromic behavior of PpQDM in malononitrile. Inset: Photographs of the corresponding PpQDM solution in malononitrile at three different sates, i.e., liquid state of the bleached (at $35^{\circ} \mathrm{C}$ ) and color return (at $100^{\circ} \mathrm{C}$ ) samples, and solid state of the color return sample (at R.T.).

Table 3.1. Amounts of FDCN, MPCT, and PpQDM in malononitrile with employed acid.

\begin{tabular}{|c|c||c|c|}
\hline $\begin{array}{c}\text { Zwitterionic } \\
\text { Chromophore }\end{array}$ & $\begin{array}{c}\text { Concentration } \\
\left(\mathbf{M}^{-1}\right)\end{array}$ & $\begin{array}{c}\text { Acid } \\
\text { (in Acetonitrile) }\end{array}$ & $\begin{array}{c}\text { Concentration } \\
\left(\mathbf{M}^{-1}\right)\end{array}$ \\
\hline FDCN & $5.700 \times 10^{-4}$ & CSA & $5.750 \times 10^{-2}$ \\
\hline MPCT & $5.700 \times 10^{-4}$ & CSA & $5.750 \times 10^{-2}$ \\
\hline PpQDM & $5.700 \times 10^{-4}$ & CSA & $5.750 \times 10^{-2}$ \\
\hline
\end{tabular}

$\mathrm{MalCN} / \mathrm{FDCN} / \mathrm{CSA}=100: 1.5 \times 10^{-2}: 2.1 \times 10^{-2}(\mathrm{w} / \mathrm{w} / \mathrm{w}) ; \mathrm{MalCN} / \mathrm{MPCT} / \mathrm{CSA}=100$ : $1.0 \times 10^{-2}: 1.3 \times 10^{-2}(\mathrm{w} / \mathrm{w} / \mathrm{w}) ; \mathrm{MalCN} / \mathrm{PpQDM} / \mathrm{CSA}=100: 1.6 \times 10^{-2}: 1.0 \times 10^{-2}(\mathrm{w} / \mathrm{w} / \mathrm{w})$.

Malononitrile is a solid at room temperature with a melting point of $30-31{ }^{\circ} \mathrm{C} .^{28}$ Therefore, during the sample preparation, the chromophores were dissolved in molten malononitrile at $\sim 35^{\circ} \mathrm{C}\left(\sim 10^{-4} \mathrm{M}\right)$. After bleaching the colored solution by adding the 
solution of CSA in acetonitrile $\left(\sim 10^{-2} \mathrm{M}\right)$, the solution was heated up to $\sim 100{ }^{\circ} \mathrm{C}$ at which the solutions were returned to the color state. Consequently, by cooling down to the ambient temperature, malononitrile was back to the solid form, which made the chromophores be partially remained in their colored state, resulting in an irreversible thermo-halochromic behavior. The irreversible thermo-halochromic behavior might be of interest and utilized for designing new thermal sensors with a permanent change from colorless to colored state. ${ }^{29}$

\subsubsection{Thermo-halochromic Properties of Zwitterionic Chromophores in a Gel Host}

PEMAGM [poly(ethylene-co-methyl acrylate-co-glycidyl methacrylate)] (Scheme 3.2) was employed as a gel host for the three zwitterionic chromophores. PEMAGM solution was prepared in 1,2-dichlorobenzene. FDCN, MPCT and PpQDM were dissolved in nitrobenzene individually. $\mathrm{BF}_{3}$ (boron trifluoride) solution was also diluted in nitrobenzene for use as an acid catalyst for epoxide ring-opening and color quencher in the system. $^{30,31}$

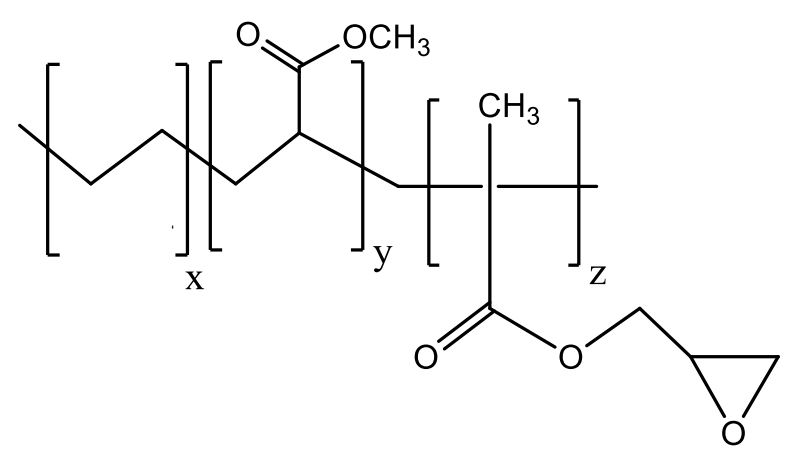

Scheme 3.2. Chemical structure of PEMAGM [poly(ethylene-co-methyl acrylate-coglycidyl methacrylate)].

Heating the bleached (colorless) mixture of polymer, chromophore and $\mathrm{BF}_{3}$ in solution up to $\sim 100{ }^{\circ} \mathrm{C}$ resulted in the formation of a crosslinked gel via acid-catalyzed 
epoxide ring-opening polymerization. ${ }^{31}$ At the same time, the color returned upon heating. The irreversible thermo-halochromic behavior for these gel materials was also observed (Figure 3.8). Going from a solution to a gel state is expected to result in restriction of the molecule motions, leading to irreversible thermo-halochromic properties. ${ }^{32}$ As previously mentioned in the case of employing malononitrile as a medium, the gel network can also be regarded as a useful medium for potential applications that require a temperature-dependent permanent color change.

(FDCN)

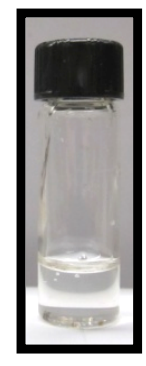

(Quenched Status) (Color Return) at R.T.

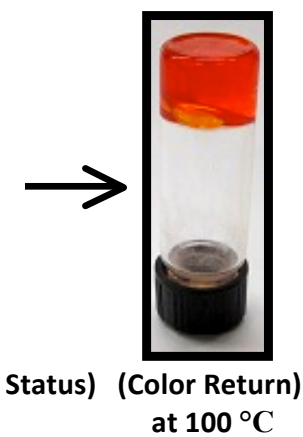

(MPCT)

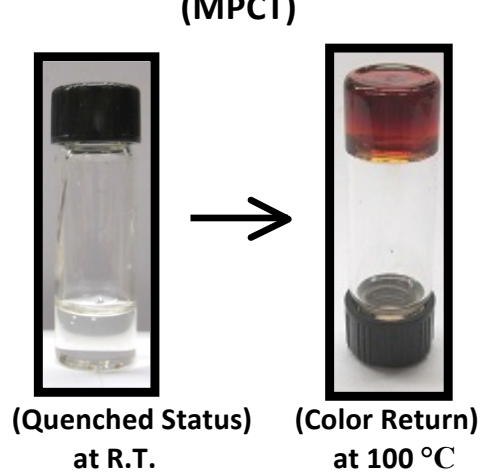

(PpQDM)

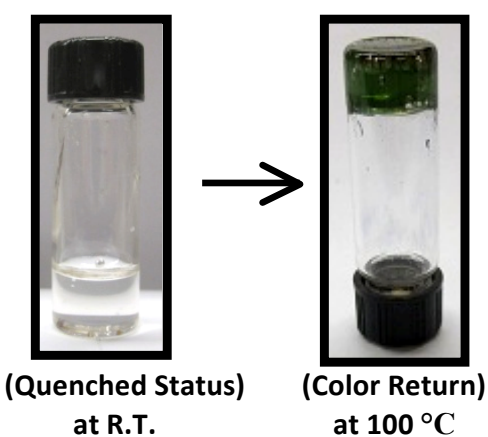

Figure 3.8. Irreversible thermo-halochromic behavior of zwitterionic chromophores in a gel host.

\subsubsection{Thermo-halochromic Properties of Zwitterionic Chromophores in Polymer}

\section{Hosts}

Regarding the increasingly attention to the polymer-based devices, polystyrene (PS) and poly(methyl methacrylate) (PMMA) (Scheme 3.3) are widely used for low cost and easy fabrication in many potential applications. ${ }^{33}$ In this regard, PS was examined as the first polymeric host for our zwitterionic chromophores. 
The chromophore solutions were added to the polymer and the prepared samples were consequently bleached via adding CSA solution. Thereafter, the bleached samples were heated. The color differences between the two states are shown in Figure 3.9. The color of all chromophore-polymer solutions reversibly changed (i.e., colorless $\leftrightarrow$ colored) by heating up to $\sim 80{ }^{\circ} \mathrm{C}$ and cooling down to the ambient temperature.

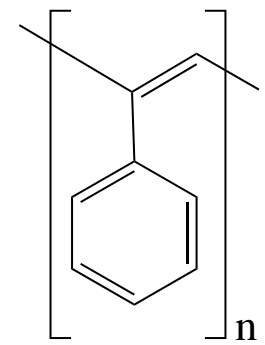

PS

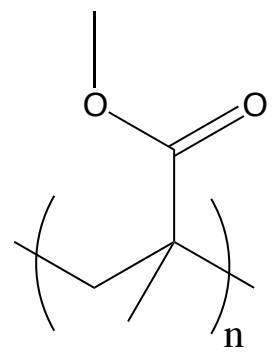

PMMA

Scheme 3.3. Chemical structures of PS and PMMA.

(FDCN)

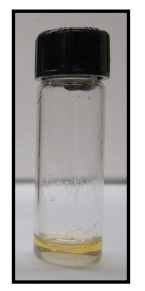

(Bleached)

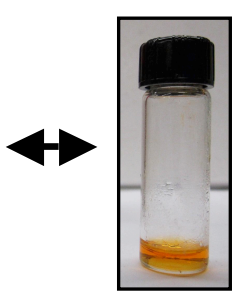

(Color Return)
(MPCT)

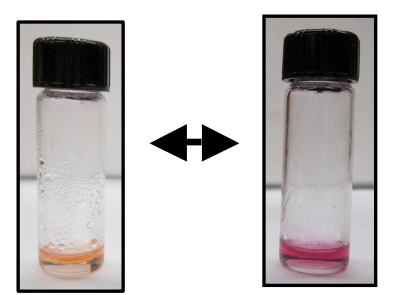

(Bleached)

(Color Return)
(PpQDM)

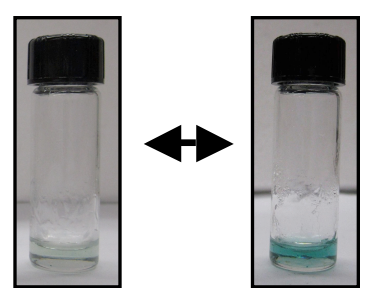

(Bleached) (Color Return)

Figure 3.9. Reversible thermo-halochromic behavior of three zwitterionic chromophores in polystyrene.

Furthermore, quantitative studies were carried out on chromophore-doped polystyrene samples (Tables 3.2). The results indicate the same trend as was previously observed in the case of the chromophores alone in solvent. PpQDM with the strongest 
proton affinity on its acceptor moiety was bleached faster by adding smaller amounts of acid, whereas the colorless state returned more readily to the color state in FDCN-doped sample via heating.

Table 3.2. Amounts of zwitterionic chromophores in polystyrene.

\begin{tabular}{|c|c|c|c|}
\hline \multicolumn{2}{|c|}{ Zwitterionic Chromophore } & Polyester (PS) & CSA \\
\hline \multicolumn{2}{|c|}{$\begin{array}{c}\text { Concentration } \\
\left(\mathbf{M}^{-1}\right)\end{array}$} & $\begin{array}{c}\text { Concentration } \\
\left(\mathbf{M}^{-1}\right)\end{array}$ & $\begin{array}{c}\text { Concentration } \\
\left(\mathbf{M}^{-1}\right)\end{array}$ \\
\hline FDCN & $7.750 \times 10^{-4}$ & $0.500 \times 10^{1}$ & $5.750 \times 10^{-2}$ \\
\hline MPCT & $7.750 \times 10^{-4}$ & $0.500 \times 10^{1}$ & $5.750 \times 10^{-2}$ \\
\hline PpQDM & $7.750 \times 10^{-4}$ & $0.500 \times 10^{1}$ & $5.750 \times 10^{-2}$ \\
\hline
\end{tabular}

PS / FDCN/ CSA = 100: $5.2 \times 10^{-2}: 10.3 \times 10^{-2}(\mathrm{w} / \mathrm{w} / \mathrm{w}) ; \mathrm{PS} / \mathrm{MPCT} / \mathrm{CSA}=100: 3.6 \times 10^{-2}$ : $6.2 \times 10^{-2}(\mathrm{w} / \mathrm{w} / \mathrm{w}) ; \mathrm{PS} / \mathrm{PpQDM} / \mathrm{CSA}=100: 5.6 \times 10^{-2}: 4.3 \times 10^{-2}(\mathrm{w} / \mathrm{w} / \mathrm{w})$.

For comparison, thermo-halochromic behavior of FDCN was studied in PS and PMMA. The optimal ratios of the polymer, chromophore and acid required for bleaching were investigated (Figure 3.10 and Table 3.3). The FDCN/CSA ratio was found to be 1:1 and 1:8 in PS and PMMA, respectively. It was significantly harder to bleach the sample in PMMA comparing to PS, which can be attributed to the proton interaction associated with the ester group of PMMA. In other words, PS does not have a hydrogen-bonding site. Therefore, PMMA and PS are two distinct hydrogen-bonding and non-hydrogen-bonding hosts, respectively. ${ }^{34}$ In addition, it was found that the chromophore could be mixed more uniformly in PS than in PMMA. The presence of these $\pi-\pi$ interactions between the guest and the host components can enhance the chromophore doping efficiency. ${ }^{35}$ Such 
interactions have been previously reported by Hunter et al., ${ }^{36}$ Jorgensen et al., ${ }^{37}$ and Tsuzuki et al. ${ }^{38}$

PS

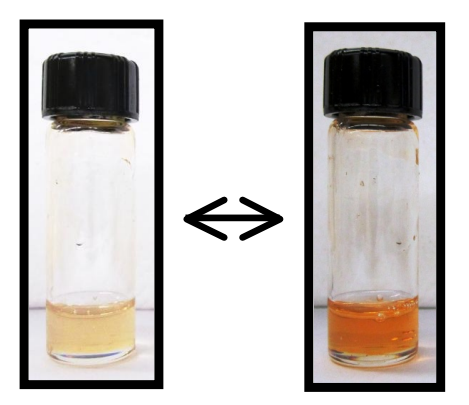

(Quenched Status) (Color Return) at R.T. at $100^{\circ} \mathrm{C}$

Figure 3.10. Thermo-halochromic behavior of FDCN in PS and PMMA.

Table 3.3. FDCN and CSA in polystyrene and poly(methyl methacrylate).

\begin{tabular}{|c|c|c||c|c||c|c|}
\hline \multicolumn{2}{|c||c||c||c||c||}{$\begin{array}{c}\text { Folymer } \\
\text { (in Chlorobenzene) }\end{array}$} & \multicolumn{2}{c|}{$\begin{array}{c}\text { CSA } \\
\text { (in Chlorobenzene) } \\
\text { (in Acetonitrile) }\end{array}$} \\
\hline \multicolumn{2}{|c|}{$\begin{array}{c}\text { Concentration } \\
\left(\mathrm{M}^{-1}\right)\end{array}$} & $\begin{array}{c}\text { Volume } \\
(\mathrm{mL})\end{array}$ & $\begin{array}{c}\text { Concentration } \\
\left(\mathrm{M}^{-1}\right)\end{array}$ & $\begin{array}{c}\text { Volume } \\
(\mathrm{mL})\end{array}$ & $\begin{array}{c}\text { Concentration } \\
\left(\mathrm{M}^{-1}\right)\end{array}$ & $\begin{array}{c}\text { Volume } \\
(\mu \mathrm{L})\end{array}$ \\
\hline PS & $0.500 \times 10^{1}$ & 0.25 & $7.750 \times 10^{-4}$ & 0.25 & $5.750 \times 10^{-2}$ & 10 \\
\hline PMMA & $0.500 \times 10^{1}$ & 0.25 & $7.750 \times 10^{-4}$ & 0.25 & $5.750 \times 10^{-2}$ & 80 \\
\hline
\end{tabular}

$\mathrm{PS} / \mathrm{FDCN} / \mathrm{CSA}=100: 5.2 \times 10^{-2}: 10.3 \times 10^{-2}(\mathrm{w} / \mathrm{w} / \mathrm{w}) ; \mathrm{PMMA} / \mathrm{FDCN} / \mathrm{CSA}=100: 5.2 \times$ $10^{-2}: 82.4 \times 10^{-2}(\mathrm{w} / \mathrm{w} / \mathrm{w})$.

Therefore, the different chromophore/CSA ratios can be attributed to the different polarity of the two polymer hosts. ${ }^{39}$ In the presence of acid, PMMA with a higher proton affinity could form the hydrogen bonding or even be protonated. ${ }^{40}$ Accordingly, without hydrogen bonding or interaction between the acid and PS, bleaching of chromophores in PS is easier and faster. 
PS-co-PMMA (Scheme 3.4) was further examined as a host for chromophores, as this copolymer may provide the characteristics that are different from the individual polymers. ${ }^{39}$ FDCN was dissolved in chlorobenzene and PpQDM, MPCT and CSA were all dissolved in $\mathrm{MeCN}$. Under the same conditions for bleaching, heating and cooling as previously done for polystyrene, the reversible thermo-halochromic behavior was also observed for each of the zwitterionic chromophores doped in PS-co-PMMA (Figure 3.11).

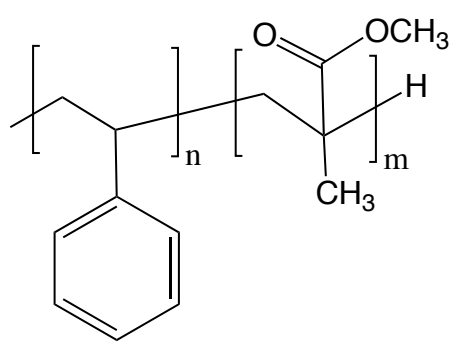

PS-co-PMMA

Scheme 3.4. Chemical structure of poly(styrene-methyl methacrylate).

MPCT, PpQDM, FDCN

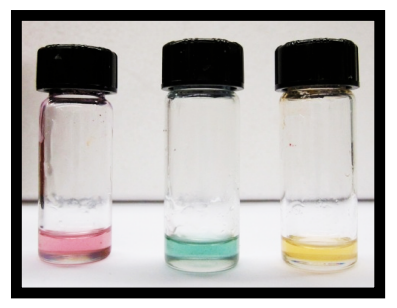

(First Bleached Status at R.T.)
MPCT, PpQDM, FDCN

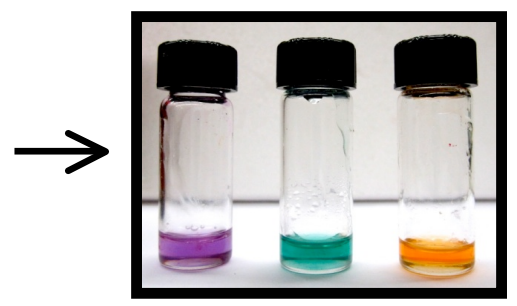

(Color Return-Heating at $80^{\circ} \mathrm{C}$ )
MPCT, PpQDM, FDCN

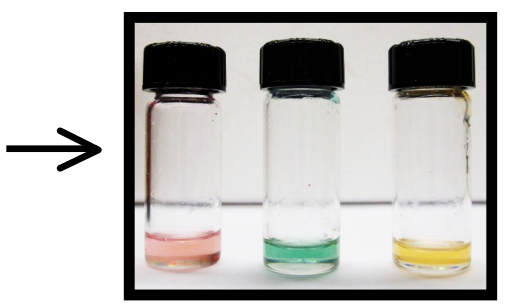

(Back to Bleached Status at R.T.)

Figure 3.11. Reversible thermo-halochromic behavior of the three zwitterionic chromophores in PS-co-PMMA.

In comparison with PS and PMMA, it is more readily to mix the chromophores in PS-co-PMMA. However, more acid and longer time were required for color bleaching in 
comparison with PS. Using PMMA as a host, the color returned with the slowest rate due to the presence of a large amount of acid used in the system. Using PS and PS-co-PMMA as a host, the thermo-halochromic process readily proceeds. PS is regarded as the most suitable host among all the polymers tested in this work.

\subsubsection{Application of Zwitterionic Chromophores as Thermo-halochromic Molecular}

\section{Trigger for Reversible Fluorescence Turn-On/Off}

The potential application of FDCN- $\mathrm{H}^{+}$complex as a molecular trigger for a reversible fluorescence turn-on/off system was initially evaluated by examining some appropriate commercial fluorescent dyes (Table 3.4).

Table 3.4. List of commercial fluorescent dyes.

\begin{tabular}{|l|c||c|c|}
\hline Fluorescent dyes & $\begin{array}{c}\text { Absorption in PhCl } \\
\left(\boldsymbol{\lambda}_{\text {max }}\right)\end{array}$ & $\begin{array}{c}\text { Excitation in PhCl } \\
\left(\boldsymbol{\lambda}_{\text {ex }}\right)\end{array}$ & $\begin{array}{c}\text { Emission in PhCl } \\
\left(\boldsymbol{\lambda}_{\text {em }}\right)\end{array}$ \\
\hline Perylene & $\begin{array}{c}\text { Peak }_{1}=413 \mathrm{~nm} \\
\text { Peak }_{2}=440 \mathrm{~nm}\end{array}$ & $410 \mathrm{~nm}$ & $\begin{array}{c}\text { Peak }_{1}=448 \mathrm{~nm} \\
\text { Peak }_{2}=476 \mathrm{~nm}\end{array}$ \\
\hline Rhodamine 6G & $530 \mathrm{~nm}$ & $490 \mathrm{~nm}$ & $548 \mathrm{~nm}$ \\
\hline Rhodamine-B & $554 \mathrm{~nm}$ & $520 \mathrm{~nm}$ & $575 \mathrm{~nm}$ \\
\hline Rose Bengal & $557 \mathrm{~nm}$ & $525 \mathrm{~nm}$ & $576 \mathrm{~nm}$ \\
\hline Crystal Violet & $596 \mathrm{~nm}$ & $350 \mathrm{~nm}$ & $414 \mathrm{~nm}$ \\
\hline
\end{tabular}

In addition to the dyes listed in the table, some other commercial fluorescent dyes were also tested, including Eosin Y, Azure (A, B, C), Acridine Orange, Indigo and Fluorescein. These dyes showed very low solubility in chlorobenzene. Eventually, perylene and Rhodamine 6G were selected for the study as their corresponding wavelengths of 
maximum absorption overlap better with the absorption profile of FDCN (i.e., $\lambda_{\max }$ of FDCN in chlorobenzene $=507 \mathrm{~nm}$ ).

\subsubsection{Fluorescence Turn-On/Off Behavior of Perylene/FDCN System}

A chlorobenzene solution of perylene $\left(3 \times 10^{-6} \mathrm{M}\right)$ was made and both fluorescence and absorption spectra were measured (Figures 3.12 and 3.13). The mixture was prepared by mixing the perylene solution with FDCN solution $\left(1.15 \times 10^{-5} \mathrm{M}\right)$. Consequently, the fluorescence spectra were recorded for the perylene/FDCN and perylene/FDCN-H $\mathrm{H}^{+}$mixtures (Figure 3.13).

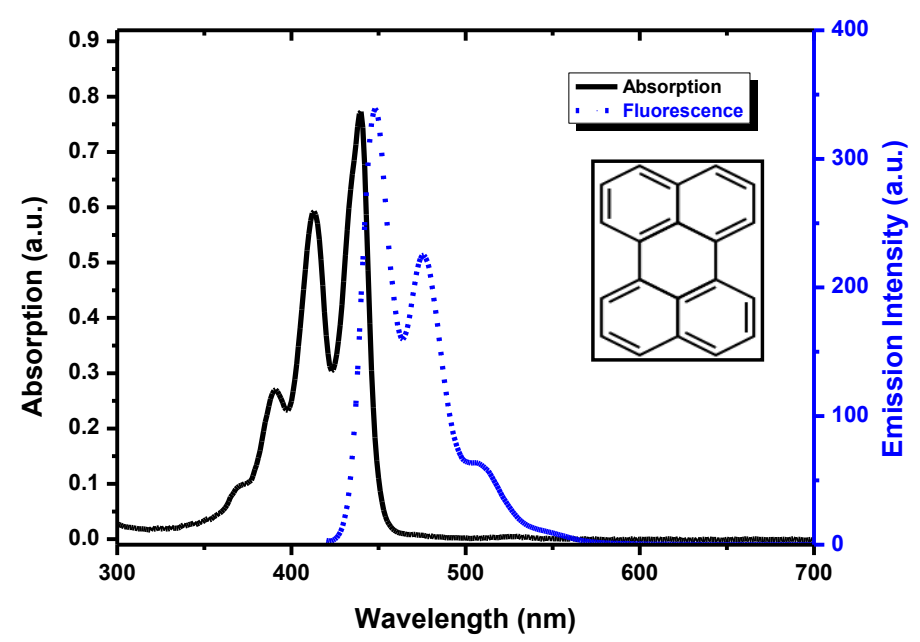

Figure 3.12. Absorption and fluorescence spectra of perylene in chlorobenzene (for emission measurement; $\lambda_{\mathrm{ex}}=410 \mathrm{~nm}$ ). 


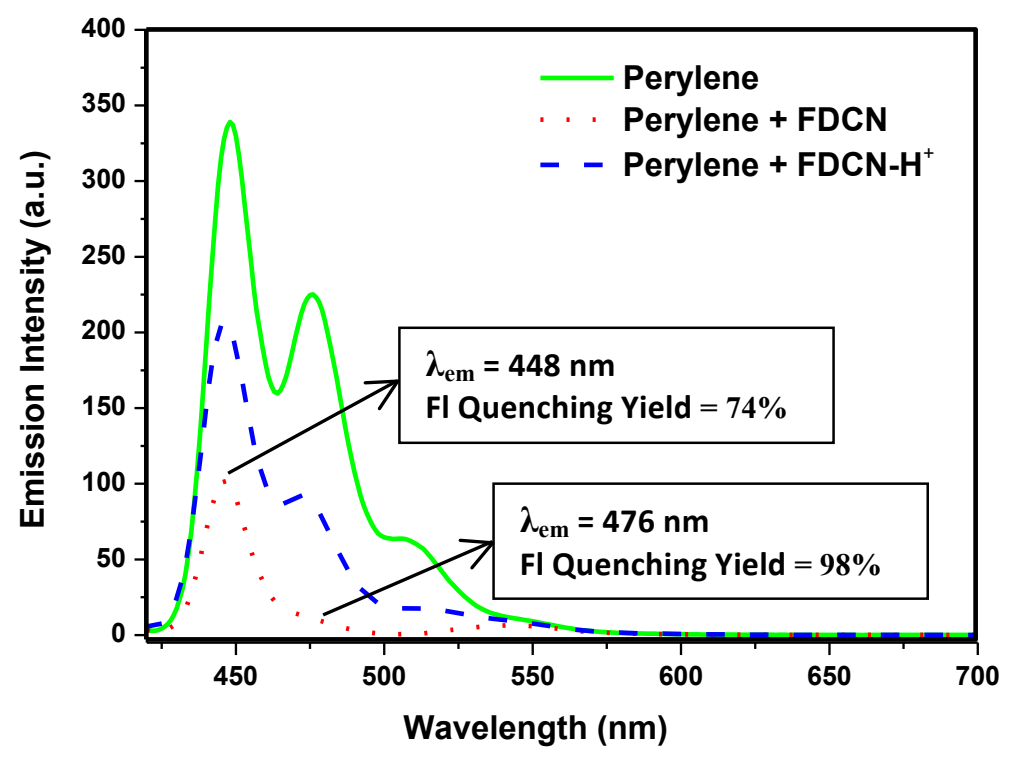

Figure 3.13. Changes in fluorescence spectra of perylene in chlorobenzene $\left(\sim 10^{-6} \mathrm{M}\right)$ via adding FDCN $\left(\sim 10^{-4} \mathrm{M}\right)$ in chlorobenzene and CSA $\left(\sim 10^{-2} \mathrm{M}\right)$ in acetonitrile.

Based on the observation, the fluorescence intensity of perylene decreased in the presence of FDCN with a $74 \%$ and $98 \%$ quenching for the emission peaks at $448 \mathrm{~nm}$ and 476 nm, respectively. Consequently, the further experiments were carried out using the second peak (i.e., $476 \mathrm{~nm}$ ) as a reference (Figure 3.13). The fluorescence quenching yield is expressed as $\left[\mathrm{I}_{0}-\mathrm{I} / \mathrm{I}_{0}(\%)\right]$, where $\mathrm{I}_{0}$ is the maximal fluorescence intensity of perylene and $\mathrm{I}$ is the intensity of the mixture of perylene and FDCN.

Thereafter, an acetonitrile solution of CSA $\left(5.75 \times 10^{-2} \mathrm{M}\right)$ was prepared and FDCNacid pair (i.e., $\mathrm{FDCN}-\mathrm{H}^{+}$) was made via adding CSA to the perylene/FDCN mixture. Fluorescence and absorption spectra of the mixture were then measured. The results showed $63 \%$ of recovery of the initial fluorescence intensity $\left(\mathrm{I}_{0}\right)$ by adding $30 \mu \mathrm{L}$ of CSA solution to the perylene/FDCN solution. At the same time, a colorimetric change was observed from 
deep-orange to pale-orange (Figures 3.14 and 3.15). The fluorescence recovery yield is calculated from the ratio of the maximal fluorescence intensity of perylene after adding acid to the initial fluorescence intensity $\left(\mathrm{I}_{0}\right)$ of perylene before adding FDCN.

When the mixture of perylene, FDCN and CSA in solution was heated up to $\sim 100{ }^{\circ} \mathrm{C}$, the deep-orange color appeared due to the release of FDCN and the fluorescence of perylene was quenched. Upon cooling to room temperature, the fluorescence turned on again to the initial level of intensity. This heating-cooling cycle was successively repeated three times and the identical results were obtained.

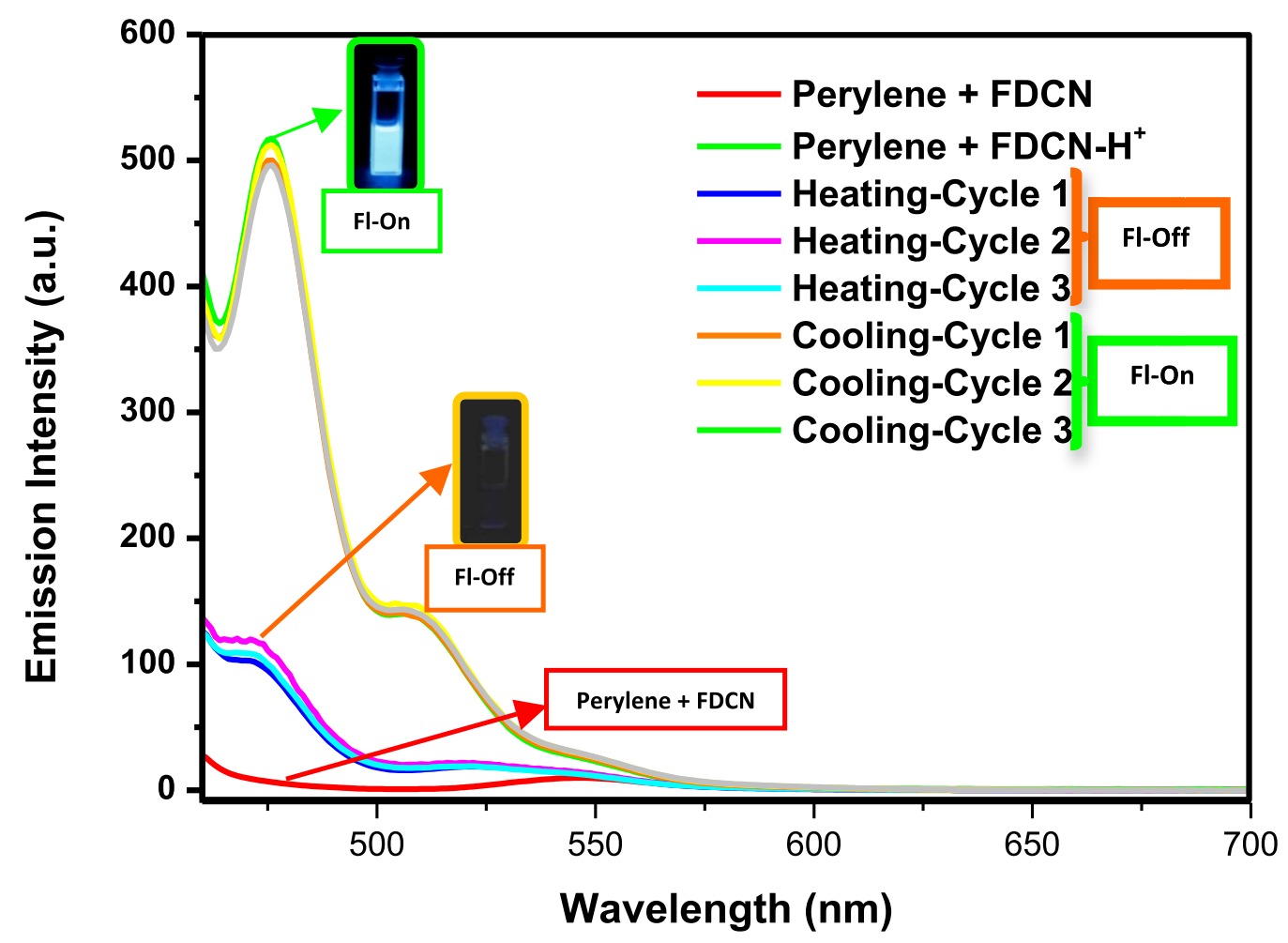

Figure 3.14. Use of FDCN- $\mathrm{H}^{+}$complex to trigger the fluorescence turn-on/off of perylene. Inset: Photographs of the on and off fluorescence emission of perylene. 


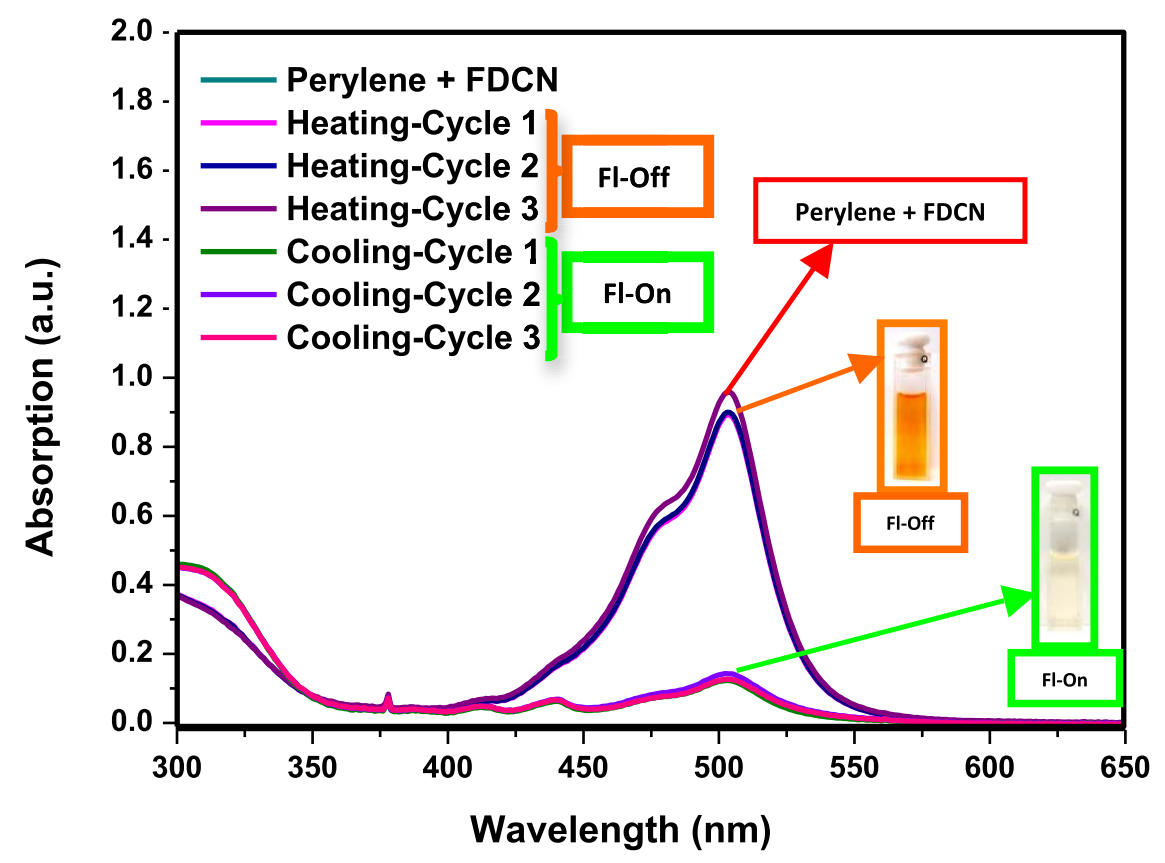

Figure 3.15. Changes in absorption spectra of perylene in chlorobenzene $\left(\sim 10^{-6} \mathrm{M}\right)$ via adding FDCN $\left(\sim 10^{-4} \mathrm{M}\right)$ in chlorobenzene and CSA $\left(\sim 10^{-2} \mathrm{M}\right)$ in acetonitrile. Inset: Photographs of the corresponding color changes.

\subsubsection{Fluorescence Turn-On/Off Behavior of Rhodamine 6G/FDCN System}

The chlorobenzene solution of Rhodamine $6 \mathrm{G}$ was made $\left(4 \times 10^{-6} \mathrm{M}\right)$ and both fluorescence and absorption spectra were measured (Figure 3.16). The Rhodamine 6G /FDCN mixture was then prepared by mixing Rhodamine 6G with FDCN in chlorobenzene $\left(1.150 \times 10^{-5} \mathrm{M}\right)$ and showed $97 \%$ quenching of fluorescence (Figure 3.17).

Upon adding $90 \mu \mathrm{L}$ of CSA $\left(5.75 \times 10^{-2} \mathrm{M}\right)$ to $3.3 \mathrm{~mL}$ of the above mixture, the fluorescence turned on to almost $84 \%$ of the initial fluorescence intensity. The same colorimetric changes were observed, going from deep-orange to pale-orange (Figures 3.18 and 3.19). 


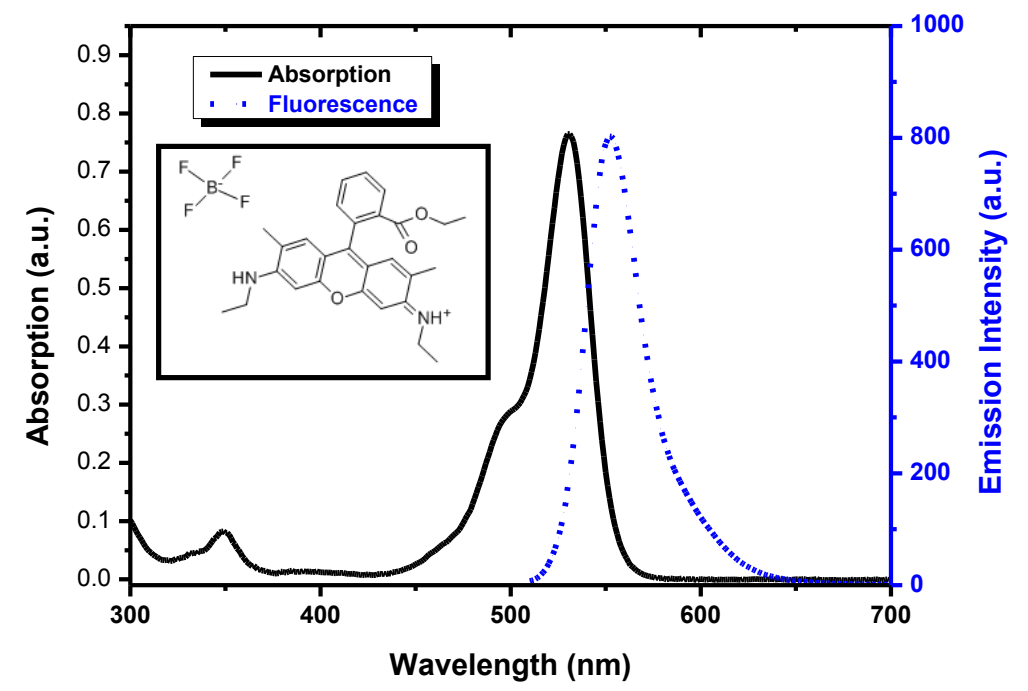

Figure 3.16. Absorption and fluorescence spectra of Rhodamine $6 \mathrm{G}$ in chlorobenzene (for emission measurement; $\lambda_{\mathrm{ex}}=490 \mathrm{~nm}$ ).

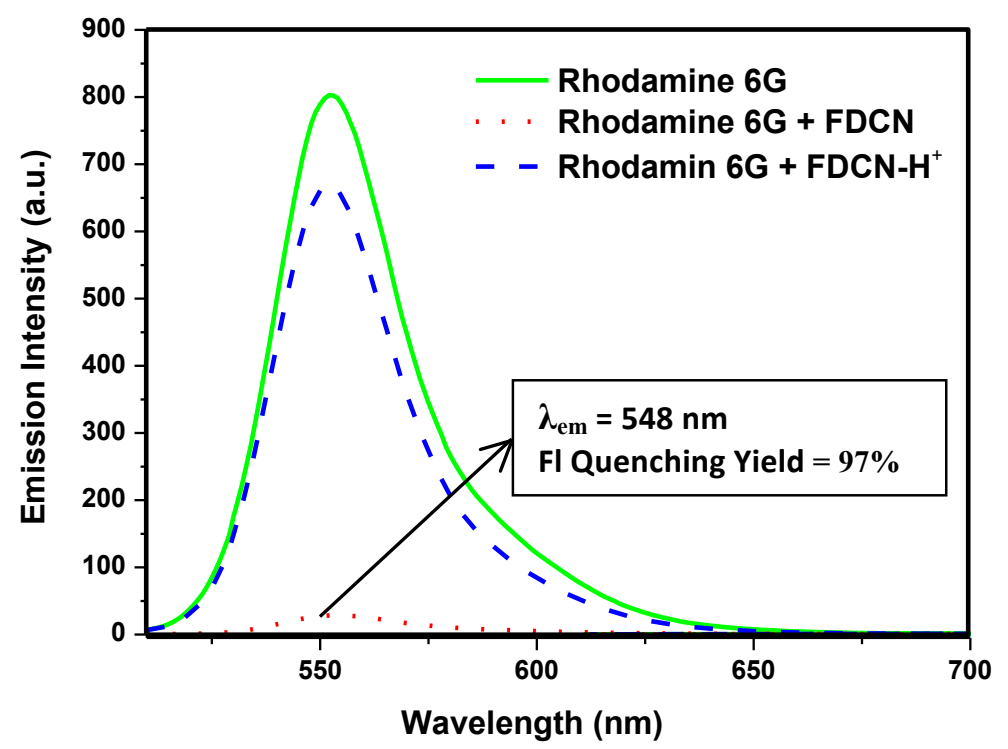

Figure 3.17. Changes in fluorescence spectra of Rhodamine $6 \mathrm{G}$ in chlorobenzene $\left(\sim 10^{-6} \mathrm{M}\right)$ via adding FDCN $\left(\sim 10^{-4} \mathrm{M}\right)$ in chlorobenzene and CSA $\left(\sim 10^{-2} \mathrm{M}\right)$ in acetonitrile. 


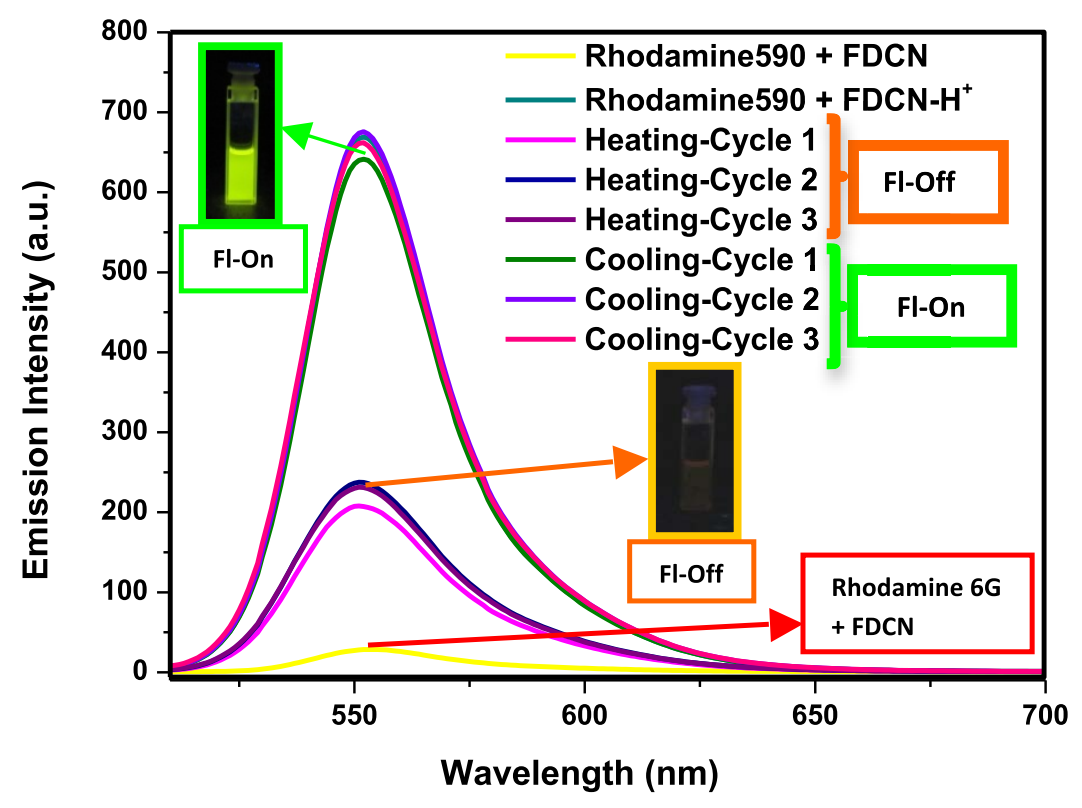

Figure 3.18. Use of $\mathrm{FDCN}-\mathrm{H}^{+}$complex to trigger the fluorescence turn-on/off of Rhodamine 6G. Inset: Photographs of the on and off fluorescence emission of Rhodamine $6 \mathrm{G}$.

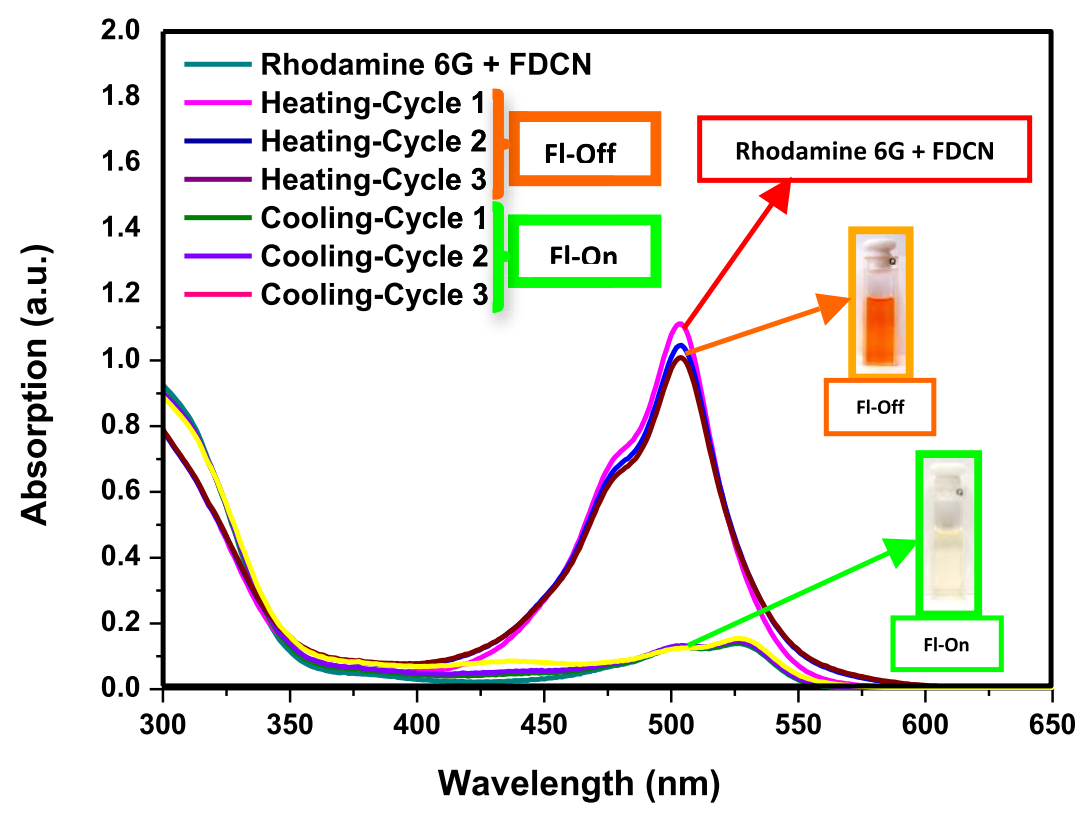

Figure 3.19. Changes in absorption spectra of Rhodamine $6 \mathrm{G}$ in chlorobenzene $\left(\sim 10^{-6} \mathrm{M}\right)$ via adding FDCN $\left(\sim 10^{-4} \mathrm{M}\right)$ in chlorobenzene and CSA $\left(\sim 10^{-2} \mathrm{M}\right)$ in acetonitrile. Inset: Photographs of the corresponding color changes. 
Moreover, it has to be also mentioned that the halochromic properties of the both commercial fluorescent dyes were initially tested by adding acid to their chlorobenzene solutions. Results showed no change of their fluorescence emissions upon adding acid, i.e. the investigated halochromic/thermo-halochromic effects were intrinsically demonstrated due to the presence of the zwitterionic chromophores.

Thereafter, the fluorescent dye/FDCN mixture could in principle work as a $\mathrm{pH}$ fluorescent sensor as well. The sensing mechanism involves the removal of the fluorescence quencher of FDCN by protonation or decrease of the $\mathrm{pH}$ in medium and subsequent fluorescence turn-on of the dye. When the $\mathrm{pH}$ value increases, FDCN is released and quenches the fluorescence of the dye. Furthermore, this same fluorescent dye/FDCN system could be utilized as an optical and colorimetric sensor to monitor the change in temperature.

\subsection{Conclusion}

The three zwitterionic chromophores are halochromic and can be made to become thermochromic. The thermochromic or reversible color-changing process is based on the protonation of the chromophore by acid and consequently deprotonation by heating. The color-changing process can be reversible and irreversible, depending on the nature of a medium. Using malononitrile and a polymer gel as a medium, thermo-halochromic process is irreversible, whereas in a suitable polymer host (i.e., PS, PMMA and PS-co-PMMA), these chromophores exhibit the reversible thermo-halochromic behavior. Furthermore, the use of FDCN (as one of the studied zwitterionic chromophore) was demonstrated as a trigger to switch on and off the fluorescence of some commercial fluorescent dyes under external stimuli of heat and $\mathrm{pH}$ changes, regarding as potential application to design new indirect thermo-indicators in future. 


\subsection{Experimental Section}

Materials. All the solvents were of spectroscopy grade (from Aldrich Chemicals) and used without further purifications. Malononitrile, PS (polystyrene), PMMA [poly(methyl methacrylate)], Poly(styrene-methyl methacrylate), PEMAGM [poly(ethylene-co-methyl acrylate-co-glycidyl methacrylate)], perylene, Rhodamine 6G tetrafluoroborate, and other examined commercial fluorescent dyes were all purchased from Aldrich Chemicals and used as received.

Methods. The UV-Vis absorption spectra were recorded on a Perklin-Elmer Lambda 900 UV-Vis-NIR spectrometer at room temperature. Fluorescence spectra were measured on a Shimadzu RF-1501 spectrofluorophotometer. The absorption and fluorescence spectra of all the samples were taken in a quartz cuvette with a path length of $10.0 \mathrm{~mm}$.

\section{Thermo-halochromic Properties of Zwitterionic Chromophores in Malononitrile.} Malononitrile was melt first and employed to dissolve the chromophores $\left(\sim 10^{-4} \mathrm{M}\right)$. After bleaching the colored solution by adding the solution of CSA in $\operatorname{MeCN}\left(\sim 10^{-2} \mathrm{M}\right)$, the solution was heated up to $\sim 100{ }^{\circ} \mathrm{C}$ to recover the color.

Thermo-halochromic Properties of Zwitterionic Chromophores in a Gel Host. The PEMAGM solution was prepared in 1,2-dichlorobenzene $(0.8 \mathrm{~g} / 10 \mathrm{~mL})$. FDCN, MPCT and PpQDM were dissolved in nitrobenzene $\left(\sim 10^{-4} \mathrm{M}\right)$ individually. $\mathrm{BF}_{3}$ (boron trifluoride) acid solution was also diluted in nitrobenzene $(0.1 \mathrm{~mL} / 10 \mathrm{~mL})$ to be employed in the system as a catalyst for crosslinking and as a color quencher.

Thermo-halochromic Properties of Zwitterionic Chromophores in Polymer Hosts. FDCN and polystyrene were dissolved in chlorobenzene. PpQDM, MPCT and CSA were all dissolved in $\mathrm{MeCN}$. The chromophore solutions were prepared individually and thereafter 
added to the polymer. Furthermore, the prepared samples were all bleached via adding CSA $\left(\sim 10^{-2} \mathrm{M}\right.$, i.e., $\left.2-6 \mu \mathrm{L}\right)$.

Fluorescence Turn-On/Off Behavior of Perylene/FDCN System. A chlorobenzene solution of perylene was made $\left(3 \times 10^{-6} \mathrm{M}\right)$. Thereafter, the perylene/FDCN mixture was prepared by mixing $2 \mathrm{~mL}$ of the perylene solution with $1 \mathrm{~mL}$ of FDCN solution $\left(1.15 \times 10^{-5}\right.$ M) which was previously prepared in the same solvent. Moreover, an acetonitrile solution of CSA $\left(5.75 \times 10^{-2} \mathrm{M}\right)$ was prepared and the titration of the perylene/FDCN mixture solution was carried out by sequentially adding $2 \mu \mathrm{L}$ of aliquots of CSA to $3 \mathrm{~mL}$ of the perylene/FDCN solution. The resulting mixture was stirred for 30 seconds at room temperature and then placed in a quartz cuvette.

Fluorescence Turn-On/Off Behavior of Rhodamine 6G/FDCN System. A chlorobenzene solution of Rhodamine $6 \mathrm{G}\left(4 \times 10^{-6} \mathrm{M}\right)$ was prepared. The Rhodamine $6 \mathrm{G} / \mathrm{FDCN}$ mixture was then prepared by mixing $2 \mathrm{~mL}$ of Rhodamine $6 \mathrm{G}$ solution with $1.5 \mathrm{~mL}$ of FDCN in chlorobenzene $\left(1.15 \times 10^{-5} \mathrm{M}\right)$. Thereafter, the previously prepared CSA solution $(5.75 \times$ $10^{-2} \mathrm{M}$ ) was added to $3.3 \mathrm{~mL}$ of Rhodamine $6 \mathrm{G} / \mathrm{FDCN}$ mixture. The resulting mixture was stirred for 30 seconds at room temperature and then placed in a quartz cuvette. 


\subsection{References}

(1) Andreu, R.; Galan, E.; Orduna, J.; Villacampa, B.; Alicante, R.; Navarrete, J. T. L.; Casado, J.; Garin, J. Chem. Eur. J. 2011, 17, 826.

(2) Reichardt, C.; Welton, T. Solvents and Solvent Effects in Organic Chemistry, fourth ed., Wiley-VCH, Weinhemin, 2011.

(3) Reichardt, C. Pure Appl. Chem. 2004, 76, 1903.

(4) Khupse, N. D.; Kumar, A. J. Phys. Chem. 2010, 114, 376.

(5) Sergio, H. A.; Alison N. J. Chem. Soc., 1996, 2, 2293.

(6) Masashi, T.; Masashi, S.; Katsuji, M.; Tadataka, Y. Dyes Pigm. 1998, 39, 97.

(7) Gageiro, V.; Aillon, M.; Rezende, M.C. J. Chem. Soc. 1992, 88, 201.

(8) Zanotto, S. P.; Scremin, M.; Machado, C.; Rezende, M. C. J. Phys. Org. Chem. 1993, $6,637$.

(9) Ramirez, C. B.; Carrasco, N.; Rezende, M. C. J. Chem. Soc. 1995, 91, 3839.

(10) Rezende, M. C.; Dominguez, M.; Aracena, A. Spectrochim. Acta 2012, 87, 61.

(11) Aitken, D.; Burkinshaw S. W., Griffiths J.; Towns, A. D. Rev. Prog. Color. 1996, 25, 1.

(12) Zhu, C. F.; Wu, A. B. J. Thermochromic Acta 2005, 425, 7.

(13) Shekhovtsov, S.V.; Mchedlov-Petrossyan, N.O.; Reichardt, C. Tetrahedron Lett. 2010, $51,4347$.

(14) Rezende, M. C.; Aracena, A. J. Spectrochimica Acta 2012, 98, 18.

(15) Seeboth, A.; Detlef, L. Thermochromic Phenomena in Polymers, Smithers Rapra Technology Publication, 2008.

(16) Seeboth, A.; Yin, C.; Kriwanek, J.; Lotzsch, D.; Schaper, R. J. Appl. Polym. Sci. 2005, 96, 1789.

(17) Naito, K. Appl. Phys. Lett. 1995, 67, 211.

(18) Naito, K. J. Mater. Chem. 1998, 8, 1379.

(19) Liu, L.; Peng, S.; Wen W.; Sheng, P. Appl. Phys. Lett. 2007, 90, 508.

(20) Yuan, L.; Lin, W.; Zheng, K.; He, L.; Huang, W. Chem. Soc. Rev. J. 2013, 42, 622.

(21) Yuan, L.; Lin, W.; Cao, Z.; Long, L. Song, J. Chem. Eur. J. 2011, 17, 689. 
(22) Hao, W. H.; McBride, A.; McBride, S.; Gao, J. P.; Wang, Z. Y. J. Mater. Chem. 2011, 21, 1040.

(23) Kramer, R. Angew. Chem. Int. Ed. 1998, 37, 722.

(24) Edelsztein, V. C.; Jares-Erijman, E. A.; Mullen, K.; Di-Chenna, P. H.; Spagnuolo, C. C. J. Mater Chem. 2012, 22, 21857.

(25) Cameraman, A.; Trotter, J. Proc. R. Soc. Lond. A. 1964, 279, 129.

(26) Zheng, H.; Zhan, X. Q.; Bian, Q. N.; Zhang, X. J. Chem. Commun. 2013, 49, 429.

(27) Meier, H.; Gerold, J.; Kolshorn, H.; Muhling, B.; Chem. Eur. J. 2004, 10, 360.

(28) Freeman, F. Chem. Rev. 1969, 69, 591.

(29) Yoon, B.; Ham, D-Y.; Yarimaga, O.; An, H.; Lee, C. W.; Kim, J-M. Adv. Mater. 2011, 23, 5492 .

(30) Coope, T. S.; Mayer, U. F. J.; Wass, D. F.; Trask, R. S.; Bond, I. P. Adv. Funct. Mater. 2011, 21, 4624.

(31) Qian, G.; Wang, Z. Y. Adv. Mater. 2012, 24, 1582.

(32) Brusatin, G.; Innocenzi, P.; Guglielmi, M.; Abbotto, A.; Beverina, L.; Pagani, G. A.; Casalboni, M.; Sarcinelli, F. J. Non-Crystalline Solids 2004, 345, 575.

(33) Li, H.; Fan, Y.; Kodzius, R.; Foulds, I. G. Microsyst. Technol. 2012, 18, 373.

(34) Zhao, Q.; Samulski E. T. Polymer 2006, 47, 663.

(35) Mizokuro, T.; Mochizuki, H.; Kobayashi, A.; Horiuchi, Sh.; Yamamoto, N.; Tanigaki, N.; Hiraga, T. Chem. Mater. 2004, 16, 3469.

(36) Hunter, C. A.; Sanders, J. K. M. J. Am. Chem. Soc. 1990, 112, 5525.

(37) Jorgensen, W. L.; Severance, D. L. M. J. Am. Chem. Soc. 1990, 112, 4768.

(38) Tsuzuki, S.; Uchimaru, T.; Matsumura, K.; Mikami, M.; Tanabe, K. Chem. Phys. Lett. 2000, 319, 547.

(39) Johnson, W. C.; Wang, J.; Chen, Z.; J. Phys. Chem. 2005, 109, 6280.

(40) Addario, V.; Guo, Y.; Chu, I. K.; Ling, Y.; Ruggerio, G.; Rodriquez, C.F.; Hopkinson, A. C.; Siu, K. W. M. Inter. J. Mass Spec. 2002, 12069, 1. 


\section{Future Work}

In future studies, methods can be designed to fabricate polymeric devices with thermo-halochromic properties (as coatings, films, fibers, etc.) via adjusting the optimal combination between the polymer component and the organic zwitterionic chromophores. This can in fact include progress in providing novel practical applications based on the investigations carried out in this research on the structure properties of the studied zwitterionic chromophores.

Considering a practical application of the thermo-halochromic behavior of the studied zwitterionic chromophores, we reported the rational design of a fluorescence turn-on/off molecular probe based on the initial investigations carried out on one of the zwitterionic chromophores in the solution phase which presented a reversible thermo-halochromism occurred based on the protonation-deprotonation process. In this regard, FDCN was candidate as the blocking agent. The intense absorption band of the synthesized zwitterionic chromophore in its UV-visible spectra and its noticeable CT were resulted in presenting competitive photophysical features making the synthesized chromophore capable of blocking the fluorescence of some commercial dyes which are considerably known for possessing high fluorescence quantum yields. In other words, the strong absorption and CT on-off character of the synthesized zwitterionic chromophores in addition to their relatively high molar extinction coefficient can make them work as a compound to block the photoluminescence of the examined commercial fluorescent dye. Accordingly, the investigated halochromism and thermo-halochromism properties of the synthesized zwitterionic chromophores in addition to their mentioned photophysical features can be all regarded as the initial steps toward the design and preparation of newly generated thermo-halochromic fluorescence turn-on/off chemical probe. 


\section{Appendix A $\quad{ }^{1}$ H NMR Spectra for MPCT Synthesis}
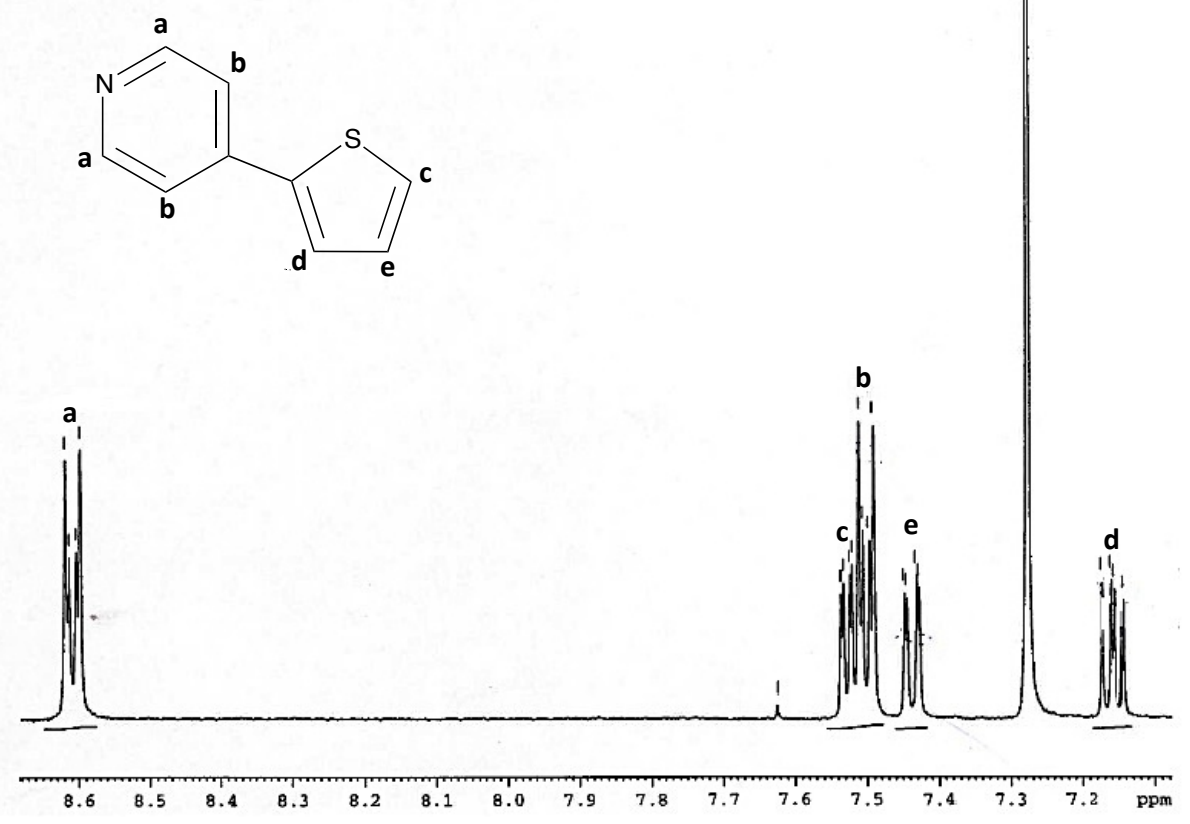

Figure S2.1. ${ }^{1} \mathrm{H}$ NMR spectrum (300 MHz) of 4-(2-thienyl)pyridine (1) in $\mathrm{CDCl}_{3}$.

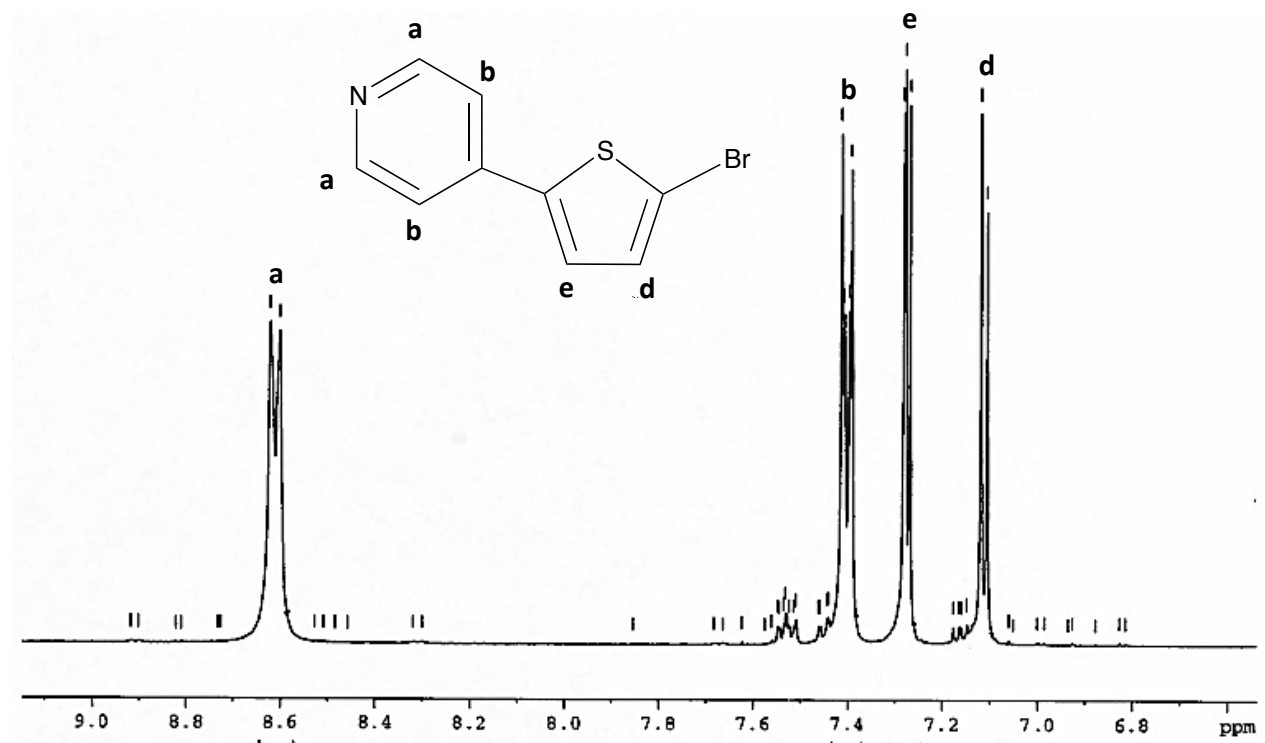

Figure S2.2. ${ }^{1} \mathrm{H}$ NMR spectrum (300 MHz) of 4-(5-bromothien-2-yl)pyridine (2) in $\mathrm{CDCl}_{3}$. 

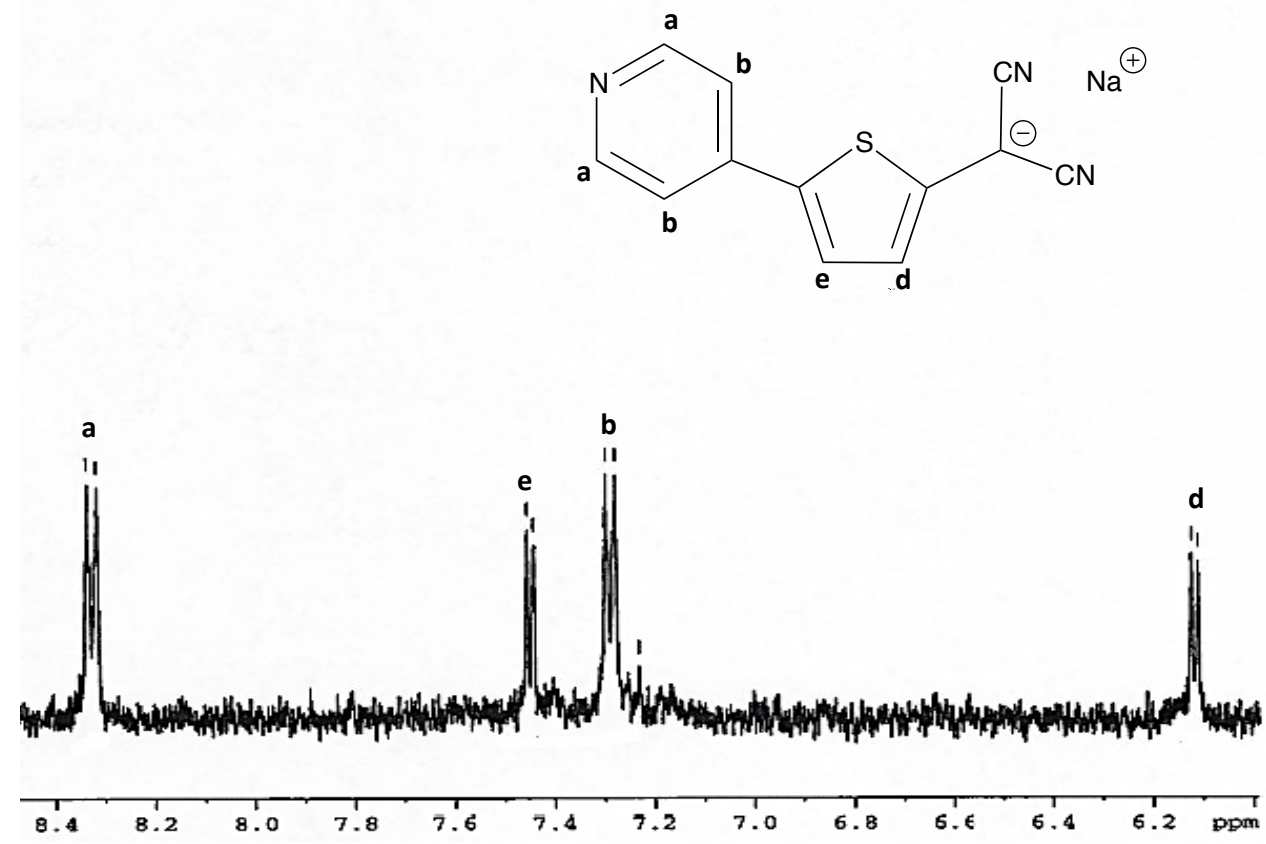

Figure S2.3. ${ }^{1} \mathrm{H}$ NMR spectrum $(300 \mathrm{MHz})$ of 4-[5-(dicyanomethanido)thien-2-yl]pyridine Sodium Salt (3) in DMSO- $d_{6}$. 